九州大学学術情報リポジトリ

Kyushu University Institutional Repository

Asymmetric evolution of eddies in rotating shallow water

\title{
荒井，正純
}

九州大学理学研究科物理学専攻

https://doi.org/10.11501/3097905

出版情報：九州大学，1994，博士（理学），課程博士 バージョン：

権利関係 : 


\section{Asymmetric evolution of eddies in rotating shallow water}

䐠 菶正細 
Asymmetric evolution of eddies in rotating shallow water

\author{
Masazumi Arai \\ Department of Physics, Faculty of Science, \\ Kyushu University
}

June 1994 


\section{Contents}

Abstract $\ldots \ldots \ldots \ldots \ldots \ldots \ldots \ldots \ldots \ldots \ldots \ldots \ldots \ldots \ldots \ldots \ldots \ldots \ldots \ldots$ iii

Part 1. Asymmetric evolution of vortices in a turbulent field ....... 1

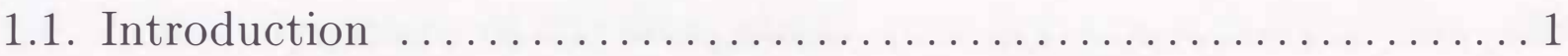

1.2. Model description

1.2.1. Shallow-water equations $\ldots \ldots \ldots \ldots \ldots \ldots \ldots \ldots \ldots \ldots \ldots \ldots \ldots \ldots \ldots \ldots \ldots$

1.2.2. Quasi-geostrophic equation $\ldots \ldots \ldots \ldots \ldots \ldots \ldots \ldots \ldots \ldots \ldots \ldots \ldots \ldots$

1.2.3. Numerical method $\ldots \ldots \ldots \ldots \ldots \ldots \ldots \ldots \ldots \ldots \ldots \ldots \ldots \ldots \ldots \ldots$

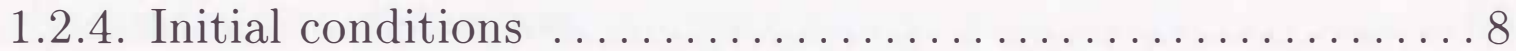

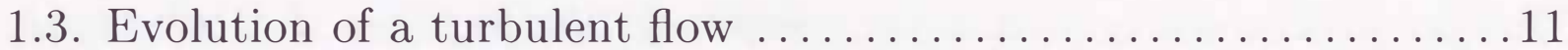

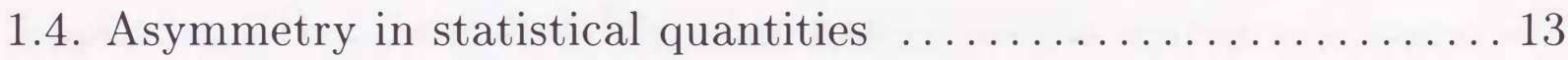

1.4.1. Energy and enstrophy transfer ................... 14

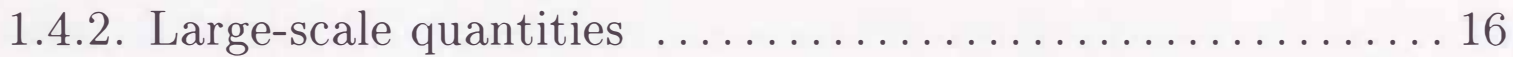

1.4.3. Small-scale quantities ...................... 19

1.5. Elongation of contours of potential vorticity $\ldots \ldots \ldots \ldots \ldots \ldots 21$

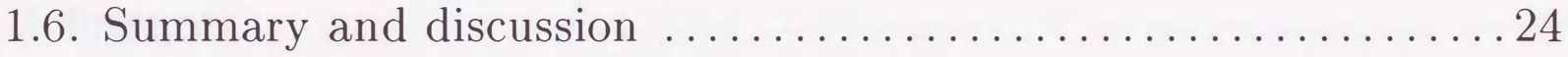

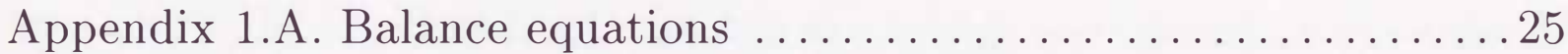

Appendix 1.B. Various geostrophic regimes $\ldots \ldots \ldots \ldots \ldots \ldots$

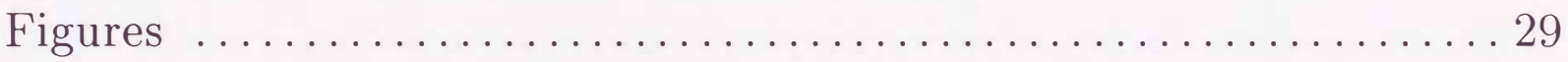

Part 2. Elongation and split-up of an isolated vortex $\ldots \ldots \ldots \ldots \ldots 40$

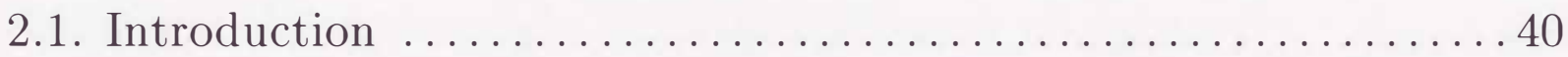

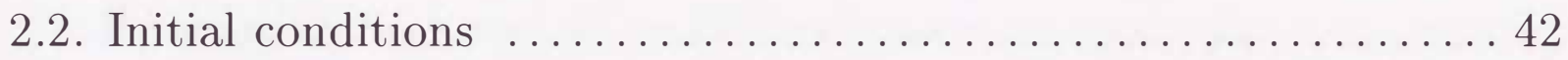

2.3. Characteristics of the evolution of an elliptical vortex ........44

2.3.1. Flow evolution .......................... 44

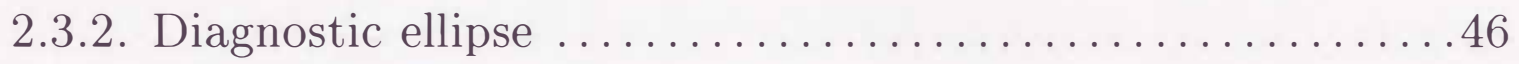

2.3.3. Dependences on the parameters $\ldots \ldots \ldots \ldots \ldots \ldots \ldots$ 
2.4. Kinematic description of the elongation and split-up .........49

2.4.1. Axisymmetrization/Elongation principle ..............49

2.4.2. Stagnation point concept $\ldots \ldots \ldots \ldots \ldots \ldots \ldots \ldots \ldots \ldots . \ldots \ldots$

2.4.3. Kinematic condition for the split-up ............... 53

2.4.4. Kinematic condition for the elongation $\ldots \ldots \ldots \ldots \ldots 56$

2.5. Linear dynamics of the elongation $\ldots \ldots \ldots \ldots \ldots \ldots \ldots \ldots \ldots$

2.5.1. Numerical method for the linear stability analysis ........ 59

2.5.2. Growth rate .................................60

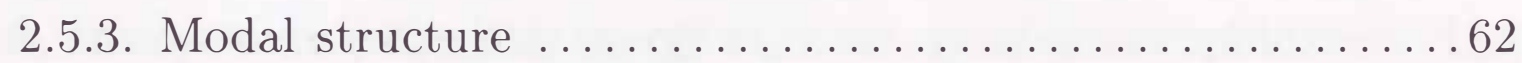

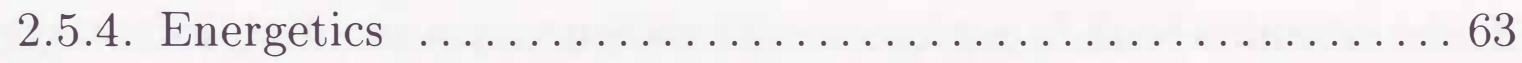

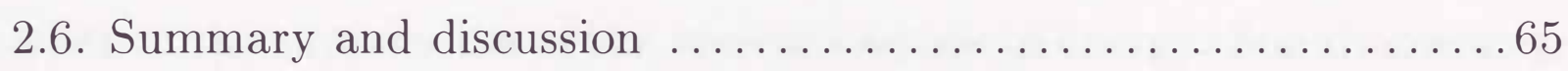

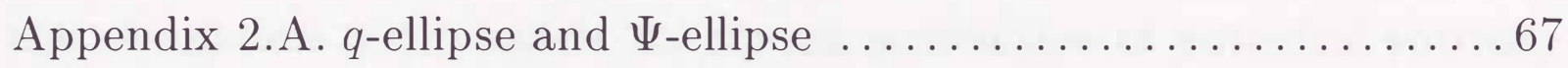

Appendix 2.B. Linear stability of an axisymmetric vortex .........6 69

Appendix 2.C. Mechanism for the axisymmetrization $\ldots \ldots \ldots \ldots . . \ldots 7$

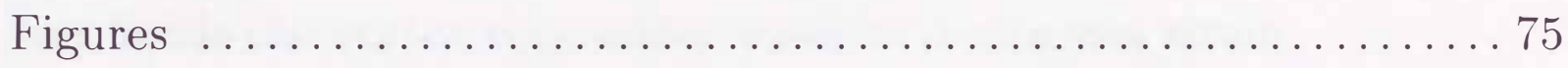

Acknowledgements .................................. 98

References ....................................... 99 


\section{Abstract}

The free evolution of many interacting vortices is investigated numerically in a rotating shallow-water system. When vertical displacements are considerably large, a remarkable asymmetric evolution is observed between cyclones and anticyclones. Equi-contours of potential vorticity of cyclones are frequently elongated, and as a result a single vortex breaks up into two vortices. Thus the merger of cyclones is suppressed. In contrast, anticyclones frequently merge as in the quasi-geostrophic case. The elongation of cyclones accompanies the stretching of fluid columns which leads to the suppression of the inverse cascade of energy. Simultaneously, the elongation accompanies the strong production of potential vorticity gradients, which leads to active enstrophy cascading. In contrast with the quasi-geostrophic case, gradients of potential vorticity are intensified just inside the vortex core where vorticity dominates strain.

The free evolution of an isolated elliptical vortex is also investigated as a prototype of an elongated vortex in a turbulent flow. It is found that the core of a cyclonic vortex splits up into two due to elongation when the maximum amplitude of vertical displacements exceeds some critical value. On the other hand, the core of a vortex eventually approaches axisymmetry for cyclones with the maximum amplitude below this value or for anticyclones with arbitrary amplitude. (Here the split-up of the vortex core is defined by the change of the sign of the Gaussian curvature of the corotating streamfunction at the center of a vortex.) A kinematic condition for the elongation/axisymmetrization is derived by considering the motion of a potential vorticity contour surrounding the stagnation point (whose type is approximately a center) at the center of a vortex. Furthermore, it is found that the split-up always occurs when the stagnation point changes into a saddle at an early stage. The time lag between 
this change and the split-up suggests an essential role of an ageostrophic motion associated with considerably thin fluid depth inside the core. The appearance of a saddle is at least a necessary condition for the split-up. The linear stability analysis shows that the elliptical vortex is unstable for a disturbance corresponding to the azimuthal wavenumber 2, the phase change of which is consistent with the kinematics of the elongation and axisymmetrization. The growth rate for large-amplitude cyclones is much larger than that for small-amplitude cyclones and arbitrary-amplitude anticyclones. Thus the growth of this disturbance is suppressed by the tendency for the axisymmetrization for the latter cases. 
Part 1. Asymmetric evolution of vortices in a turbulent field

\subsection{Introduction}

Two-dimensional turbulence has received theoretical attentions, since the two-dimensional Euler equations (equivalent to the non-divergent quasi-geostrophic equation) have an additional integral invariant, enstrophy besides energy in contrast with the three-dimensional equations (e.g., Kraichnan and Montgomery 1980). These two constraints lead to the inverse cascade of energy and the cascade of enstrophy through nonlinear interactions. A typical feature of two-dimensional turbulence is the predominance of coherent vortices which have a lifetime greatly exceeding their internal circulation time scale (McWilliams 1984). In a turbulent flow, vorticity contours tend to be statistically elongated. The elongated contours are folded or wrapped around cores with relatively stronger vorticity, and several coherent vortices develop in a background sea of passive filamentary debris. After coherent vortices are well established, two like-signed vortices coalesce into a larger single vortex successively. The elongation of vorticity contours is a manner of the cascade of enstrophy, and the wrapping of vorticity contours and the merger of like-signed vortices are manners of the inverse cascade of energy in physical space.

From a practical point of view, two-dimensional turbulence has relevance to large-scale oceanic and atmospheric turbulence (i.e., geostrophic turbulence). In actual geophysical flows, however, the effect of horizontal divergence cannot be neglected. Under the assumption of weak vertical motions (i.e., weak horizontal divergence), such flows are governed by the quasi-geostrophic equation (see the Appendix 1.B). In this case, potential vorticity determines a flow field instead of vorticity. The influence of the strain induced by a distribution of potential vorticity is weaken in the distance as the horizontal scale of the distribution compared with the 
Rossby radius of deformation increases. Thus during the merger of two vortices, filamentation of contours of potential vorticity is less remarkable (Polvani et al. 1989). The effect of divergence also decreases the rate of the energy and enstrophy transfer in wavenumber space (Larichev and McWilliams 1991).

The quasi-geostrophic approximation with neglect of the horizontal mass divergence introduces artificial symmetry between cyclonic and anticyclonic flows which is not present in the original shallow-water system. This simplification is, however, inappropriate for oceanic submesoscale or mesoscale vortices, since these vortices are accompanied by considerable vertical displacements of equi-density surfaces (Robinson 1983). In addition, anticyclones are much more frequently observed than cyclones in some areas of oceans (McWilliams 1985). Here we note that the mesoscale is comparable to or a few times larger than the internal radius of deformation (about $50 \mathrm{~km}$ in mid-latitude oceans), while the submesoscale is less than this radius.

To remedy the limitations of the quasi-geostrophic approximation, several types of geostrophic equations have been proposed by freeing the assumption of weak vertical displacements. Yamagata (1982) derived the intermediate geostrophic equation with a scalar nonlinearity. Williams and Yamagata (1984) and Cushman-Roisin (1986) derived the frontal geostrophic equation with a cubic nonlinearity. These terms break the symmetry between cyclones and anticyclones. Pavia and CushmanRoisin (1990) investigated the merger of two vortices using the frontal geostrophic equation, and showed that cyclones are resistant to merging while anticyclones are eager to merge. Each type of geostrophic equations is, however, valid only in a particular dynamical regime, thus cannot describe interactions between scales in different dynamical regimes as in cascading processes. We note that the frontal geostrophic equation is 
valid when $\varepsilon \sim F^{-1} \ll 1$, where $\varepsilon$ and $F$ are the Rossby number and the rotational Froude number, respectively (see the Appendix 1.B for its derivation).

The above disadvantage of each geostrophic equation is partly overcome by using a general geostrophic equation. Cushman-Roisin and Tang $(1989,1990)$ investigated the free evolution of many interacting vortices using the general geostrophic equation on a $\beta$-plane. They showed a remarkable asymmetry between cyclones and anticyclones when the typical horizontal scale of vortices is beyond the radius of deformation. Namely, the emerging vortices in a turbulent flow are all anticyclonic, while cyclonic vortices are disintegrated and become a smaller-scale turbulent background. Cushman-Roisin and Tang (1990) also investigated the evolution of an isolated axisymmetric vortex on an $f$-plane. They showed that the cyclonic vortex with strong vertical displacements is unstable and breaks up into a number of smaller vortices while its anticyclonic counterpart is stable. They suggested using the variational method that the instability of such cyclones is attributed to the cubic nonlinearity. But physical explanations for the break-up have not been presented yet. Note that the cubic nonlinearity is the only symmetry-breaking term between anticyclones and cyclones on an $f$-plane.

The above findings on the $f$-plane are, however, surprising, and raise some questions. The frontal geostrophic equation on the $f$-plane conserves potential energy and kinetic energy separately, where the former corresponds to total energy and the latter to potential enstrophy in the quasi-geostrophic and shallow-water equations. Thus the cascade of potential energy will be inhibited, which demonstrates that the break-up of a vortex into many smaller vortices is implausible. In contrast to these equations, the general geostrophic equation conserves only total energy and does not conserve potential enstrophy even on the $f$-plane (Hukuda 
and Yamagata 1988). This comes from that the general geostrophic equation is a hybrid of the quasi-geostrophic and frontal geostrophic equations. Therefore the general geostrophic equation will not provide a good description of cascading processes. In addition, geostrophic equations are inappropriate when centrifugal force cannot be negligible as some cases of oceanic vortices. The above problems motivate the author to adopt the original shallow-water equations as a model system.

Investigations about turbulence in the rotating shallow-water equations have been very scarce, and the effects of strong vertical displacement and centrifugal force have not been revealed yet. Farge and Sadourny (1989) investigated the interaction between the rotational and divergent components of motion depending on the level of the divergent component. Although their work is restricted to the cases with weak vertical displacements and has nothing to do with the asymmetric properties, their following findings have relevance to the present interests about energy transfer properties: At small scales, the rotational component behaves as if the flow is non-divergent, while an intense cascade of energy occurs within the divergent component; At large scales the inverse cascade of energy of the rotational component is reduced when the level of the energy of the divergent component is high. The mechanism for the energy cascade of the divergent component is very clear when the vertical displacement is very weak. Since potential vorticity is approximately zero for small-amplitude inertial-gravity waves, the enstrophy conservation does not work.

In the first part of the present work, the free evolution of many interacting vortices is investigated numerically in a rotating shallow-water system. The purpose is to clarify asymmetric natures between cyclonic and anticyclonic vortices when the vertical displacement is strong and the centrifugal force is not always negligibly small. Since an uniformlyrotating frame (an $f$-plane) is adopted, nature of asymmetry is quite 
different from that due to the scalar nonlinearity. In the frontal geostrophic regime, the symmetry breaking term is expressed in the form of the cubic nonlinearity. However, outside the frontal geostrophic regime, this term will not be expressed in such a simple form. Thus the flow evolution in the latter regime will be different from that in the former regime.

\subsection{Model description}

\subsubsection{Shallow-water equations}

We consider a frame uniformly rotating in the counter-clockwise direction with the angular velocity $f / 2$ (i.e., on an $f$-plane). The velocity, horizontal scale, time, fluid depth and free surface displacement are scaled by $U, L, L / U, H$ and $f L U / g$, respectively, where $H$ is the undisturbed fluid depth and $g$ is the acceleration due to gravity. Here $H$ is assumed to be constant. The non-dimensional shallow-water equations are

$$
\begin{aligned}
& \varepsilon \frac{D \boldsymbol{u}}{D t}+\hat{z} \times \boldsymbol{u}+\nabla \eta=-\varepsilon \nu \triangle^{2} \boldsymbol{u}, \\
& \frac{\partial h}{\partial t}+\nabla \cdot(h \boldsymbol{u})=0
\end{aligned}
$$

where $\boldsymbol{u}=(u, v)$ is the horizontal velocity vector, $\eta$ is the free surface elevation (or the interface depression if a reduced gravity model is adopted), $h=1+\varepsilon F \eta$ is the fluid depth, $\hat{z}$ is the vertical unit vector parallel to the axis of rotation, $\nabla$ and $\triangle$ are the horizontal gradient and Laplacian operators, respectively, and $\frac{D}{D t}=\frac{\partial}{\partial t}+(\boldsymbol{u} \cdot \nabla)$ is the Lagrangian derivative (e.g., Pedlosky 1987). Here the hyperviscosity is introduced to dissipate excitations at very small scales without affecting flows at large scales, and $\nu$ is the hyperviscosity coefficient. The above equations have two nondimensional parameters, the Rossby number $\varepsilon=U / f L$, and the rotational Froude number $F=\left(L / L_{R}\right)^{2}$, where $L_{R}=\sqrt{g H} / f$ is the Rossby radius of deformation based on the undisturbed depth $H$. Here we introduce an 
additional parameter $\delta=\varepsilon F$ which measures the vertical scale.

The potential vorticity $q=(1+\varepsilon \zeta) / h$ yields

$$
\frac{D q}{D t}=-\varepsilon \nu \frac{\triangle^{2} \zeta}{h}
$$

where $\zeta$ is the vertical component of vorticity defined as

$$
\zeta=\frac{\partial v}{\partial x}-\frac{\partial u}{\partial y}
$$

The above equation implies that $q$ is a Lagrangian invariant in an inviscid case. In a steady state, the transport streamfunction $\Psi$ can be introduced as

$$
h \boldsymbol{u}=\hat{z} \times \nabla \Psi
$$

In terms of this, (1.2) is reduced to $J(\Psi, q)=0$ in an inviscid case, where $J$ is the Jacobian operator defined by $J(A, B)=\frac{\partial A}{\partial x} \frac{\partial B}{\partial y}-\frac{\partial A}{\partial y} \frac{\partial B}{\partial x}$. Thus $q$ is a function of $\Psi$, that is, $\Psi$ distributions coincide with $q$ distributions in an inviscid steady state.

The velocity can be decomposed as

$$
\boldsymbol{u}=\hat{z} \times \nabla \psi+\nabla \phi
$$

where $\psi$ is the streamfunction and $\phi$ is the velocity potential. By taking the rotation and divergence of (1.1a), the equations for the vorticity and divergence are obtained as follows:

$$
\begin{aligned}
& \varepsilon\left(\frac{\partial \zeta}{\partial t}+J(\psi, \zeta)\right)+d+\varepsilon(\nabla \phi \cdot \nabla \zeta+\zeta d)=-\varepsilon \nu \triangle^{2} \zeta \\
& \varepsilon\left(\frac{\partial d}{\partial t}+J(\psi, d)\right)+\varepsilon \nabla \phi \cdot \nabla d+\triangle \eta_{a}+\varepsilon\left(s_{i j}^{2}-\frac{1}{2} \zeta^{2}\right)=-\varepsilon \nu \triangle^{2} d,
\end{aligned}
$$

where $d=\nabla \cdot \boldsymbol{u}$ is the horizontal divergence, $\eta_{a}=\eta-\psi$ is the ageostrophic elevation, $s_{i j}$ is the $(i, j)$-component of the rate-of-strain tensor defined as

$$
s_{11}=\frac{\partial u}{\partial x}, \quad s_{22}=\frac{\partial v}{\partial y}, \quad s_{12}=s_{21}=\frac{1}{2}\left(\frac{\partial v}{\partial x}+\frac{\partial u}{\partial y}\right)
$$


and a summation over repeated indices is assumed. Here we examine the transformation to reverse the polarity of a vortex as $\psi \rightarrow-\psi, \phi \rightarrow-\phi$, $\eta_{a} \rightarrow \eta_{a}$ and $(x, y) \rightarrow(x,-y)$. In the vorticity equation (1.5a), the terms in the first bracket and $d$ on the left-hand side are symmetric, while those in the second bracket break the symmetry. In the divergence equation (1.5b), two terms in the first bracket on the left-hand side are symmetric, while the third and fourth terms break the symmetry. The term $s_{i j}^{2}$ behaves in a complicated manner. The continuity equation (1.1b) also behaves in a complicated manner, since the components $\psi$ and $\eta_{a}$ of $\eta$ are transformed in different ways. The above facts demonstrate that the shallow-water equations are asymmetric between cyclonic and anticyclonic solutions.

\subsubsection{Quasi-geostrophic equation}

When the Rossby number is small and the rotational Froude number $F$ is finite, that is, when the amplitude parameter $\delta=\varepsilon F$ is small, the shallow-water equations (1.1a) and (1.1b) are reduced to the quaigeostrophic equation as described in the Appendix 1.B. With the hyperviscosity, the quasi-geostrophic equation is

$$
\frac{\partial q}{\partial t}+J(\psi, q)=-\nu \triangle^{3} \psi
$$

Here $\psi$ is the streamfunction, and $q$ is the potential vorticity defined by

$$
q=(\triangle-F) \psi
$$

Under the transformation, $\psi \rightarrow-\psi$ and $(x, y) \rightarrow(x,-y)$, the quasigeostrophic equation is invariant. This demonstrates that the quasigeostrophic equation is symmetric between cyclonic and anticyclonic solutions in contrast with the original shallow-water equations. 


\subsubsection{Numerical method}

We consider a square domain of the half width $W$ in the $(x, y)$ plane $(|x|,|y| \leq W)$ with doubly-periodic boundary conditions. On this domain, any field variable can be expressed in a double Fourier series. Thus the shallow-water equations, (1.1a) and (1.1b) are amenable to a pseudospectral method. After transforming the field variables to physical space, the nonlinear terms are computed at the grid points. Then the nonlinear terms are transformed back to wavenumber space, and the time-marching is done using a leap-frog scheme with the Crank-Nicolson scheme for the dissipation term. The second-order Runge-Kutta scheme is used every 20 time steps to suppress the time-splitting instability. To remove aliasing errors completely, the Fourier series is truncated at a wavenumber $\frac{\pi}{W} M$ in each direction, where $M=[N / 3], N$ is the number of grid points in each direction and $[\cdots]$ denotes the integer part. Here the circular truncation is imposed for the sake of isotropy at smaller scales.

The time step $\Delta t$ is chosen to resolve inertial-gravity waves as $\Delta t=$ $\Delta x \cdot c \cdot \varepsilon \sqrt{F}$, where $\Delta x=2 W / N$ is the grid spacing, $(\varepsilon \sqrt{F})^{-1}$ is the nondimensional phase speed of the inertial-gravity wave and $c$ is an appropriate constant between 0.2 and 0.3 .

The quasi-geostrophic equation (1.6) is also solved by the similar method, except that the time-marching is done using the fourth-order Runge-Kutta-Gill scheme.

\subsubsection{Initial conditions}

Since many Fourier components are simultaneously present in a turbulent evolution, the horizontal scale is traditionally non-dimensionalized in terms of the size of the domain. Thus the half size of the domain is chosen to be $W=\pi$ here. The initial velocity field is assumed to be in gradient-wind balance. Under this assumption, the irrotational com- 
ponent of velocity and the surface displacement can be obtained by the balance equations after the rotational component is specified (Gent and McWilliams 1983; Norton et al. 1986). The derivation of the balance equations is described in the Appendix 1.A. The wavenumber $\left(k_{x}, k_{y}\right)\left(k_{x}\right.$ and $k_{y}$ are integers between $-M$ and $M$ ) spectrum of the streamfunction is given by

$$
\tilde{\psi}\left(k_{x}, k_{y}\right)=\psi_{0} \exp \left[-\left(\frac{k-k_{0}}{0.6}\right)^{2}+i \theta_{k_{x} k_{y}}\right],
$$

where $k=\sqrt{k_{x}^{2}+k_{y}^{2}}$, and $\theta_{k_{x} k_{y}}$ is a random phase between 0 and $2 \pi$. The above spectrum shows a sharp peak at $k=k_{0}$. The amplitude factor $\psi_{0}$ is chosen such that $\left\langle\psi^{2}\right\rangle=6 \times 10^{-3}$ initially, where $\langle\cdots\rangle$ denotes the spatial average over the full domain. The surface elevation is computed using (1.A1b), where its spatial average is set at zero. The velocity potential is computed using the simplified equation of (1.A4), where the last term on the right-hand side is neglected since this term is $O\left(\varepsilon^{2}\right)$.

Three experiments (Case I, Case II and Case III) are conducted for different combinations of the amplitude parameter $\delta$ and the rotational Froude number $F$ as listed in Table 1.1. Here we note that Case I is initially in the quasi-geostrophic regime, while Case III is in the frontal geostrophic regime. The Rossby number $\varepsilon$ for Case II is not so small that the geostrophic approximation is inappropriate. The initial peak wavenumber is fixed at $k_{0}=4$ for all experiments, thus there exist nearly 16 cyclones and 16 anticyclones initially as shown in Fig. 1.1. Because of the choice of the very narrow-band spectrum, we can specify initial vortices with similar horizontal and vertical scales. To check initial value dependences for an anticipated asymmetric evolution, another experiment (Case II') is conducted using the initial values obtained by the transformation of the initial $\psi$ of Case II as $(x, y) \rightarrow(x,-y), \psi \rightarrow-\psi$. (Here a smaller value of $\delta$, i.e., $\varepsilon$ is chosen such that the fluid depth is 


\begin{tabular}{lcccccc}
\hline \hline & $\delta$ & $F$ & $\varepsilon$ & $\delta_{*}$ & $F_{*}$ & $\varepsilon_{*}$ \\
\hline Case I & 0.37 & 16 & 0.023 & 0.029 & 1 & 0.029 \\
Case II & 3.7 & 16 & 0.23 & 0.29 & 1 & 0.29 \\
Case II' & 2.9 & 16 & 0.18 & 0.22 & 1 & 0.22 \\
Case III & 4.5 & 144 & 0.03 & 0.35 & 9 & 0.04 \\
\hline \hline
\end{tabular}

Table 1.1. Parameters for each simulation of the turbulent evolution.

positive throughout the domain.) The grid points $N$ in each direction is 256. This resolution is satisfactory enough, since the results for $N=128$ are qualitatively similar to those for $N=256$. The hyperviscosity coefficient is fixed at $\nu=8.0 \times 10^{-8}$, which is an appropriate value to achieve smooth contours of potential vorticity throughout the evolutions.

The non-dimensional parameters defined above, however, do not represent typical scales of initial vortices. Thus it is preferable to rescale the horizontal scale by the characteristic scale $k_{0}^{-1}$ of vortices and the velocity scale by $k_{0} \psi_{*}$, where $\psi_{*}=\sqrt{\left\langle\psi^{2}\right\rangle}$. Then the rescaled parameters, $\varepsilon_{*}, F_{*}$ and $\delta_{*}$ are related to the old parameters $\varepsilon, F$ and $\delta$ as $\varepsilon_{*}=k_{0}^{2} \psi_{*} \varepsilon, F_{*}=k_{0}^{-2} F$ and $\delta_{*}=\psi_{*} \delta$, respectively. Those parameters for each experiment are also listed in Table 1.1.

The departures from rotational flow have been examined by the ratio $r_{d}$ of the root-mean-square value of the divergent velocity $\nabla \phi$ to that of the total velocity $\boldsymbol{u}$ defined as

$$
r_{d}=\frac{\sqrt{\langle\nabla \phi \cdot \nabla \phi\rangle}}{\sqrt{\langle\boldsymbol{u} \cdot \boldsymbol{u}\rangle}} .
$$

For Case I, $r_{d}$ was within $0.4 \%$ throughout the evolution. For Case II, $r_{d}$ was $2.3 \%$ at $t=0$ and reached the maximum value $4.6 \%$ at $t=28.3$. Thus even in the case of strong vertical displacements, the rotational motion 
is dominant throughout the evolution.

The stability of the numerical scheme has been checked in the inviscid equations for Case II. The total energy fluctuated within $0.03 \%$ and the potential enstrophy decreased to only $0.7 \%$, respectively of its initial value by $t=50$.

\subsection{Evolution of a turbulent flow}

In order to have an overview of an asymmetric evolution between cyclones and anticyclones, we first show the evolution of contours of potential vorticity $q$. From the definition $q=(1+\varepsilon \zeta) /(1+\delta \eta)$, cyclonic flows $(\eta<0, \zeta>0)$ correspond to $q>1$, and anticyclonic flows $(\eta>0, \zeta<0)$ correspond to $q<1$.

First we show the cases with $F_{*}=1$. Figure 1.1(a) shows the evolution for Case I. Since the vertical displacement is very small, the evolution will be well governed by the quasi-geostrophic equation. After $t \sim 16$, remarkable elongation of $q$ contours is frequently observed which mainly accompanies the formation of large-scale vortices by following manners. Two like-signed vortices with comparable strength tend to merge (e.g., in the upper-left corner from $t=16$ to $t=24$, and in the middle-lower part from $t=28$ to $t=32$ ). Tails of elongated contours are wound up around another stronger vortices (in the middle-left part from $t=16$ to $t=20$, and in the lower-right part at $t=16)$. Elongated vortices spontaneously wind up around their centers (not shown). Very weak vortices are passively elongated, and torn away or dissipated (around the middle-lower part from $t=24$ to $t=32$ ). As expected, no notable differences are observed between cyclones and anticyclones.

Figure 1.1(b) shows the evolution for Case II. Since the vertical displacement is considerably large, we expect asymmetry between cyclones and anticyclones. The initial $q$ distribution is actually quite different be- 
tween cyclones and anticyclones. We note that the contour interval for $q>1$ is more than 10 times larger than that for $q<1$. At the early stage, overall shapes of cyclones are distorted from initial elliptical or nearly circular shapes to shrunk triangular shapes (see $t=4$ ). After $t \sim 12, q$ contours of cyclones are frequently elongated into filamentary structures. Typical three events are observed in the figure. The first event (Event 1) occurs from $t=16$ to $t=20$ near the upper-right area, where the partially-split vortex cores coalesce again. The second event (Event 2) occurs from $t=20$ to $t=24$ near the middle-left area, where the elongated filament rolls up. The third event (Event 3) occurs from $t=24$ to $t=28$ near the lower-right area, where the elongated vortex breaks up into two vortices. Not only the elongation suppresses the merger of two cyclones, but the merger itself seems to be halted as pointed out by Pavia and Cushman-Roisin (1990). In contrast, anticyclones behave in similar manners to vortices in Case I. The above significant asymmetric features are also observed in Case II' (not shown).

Next we show the case with $F_{*}=9$ (Case III) in Fig. 1.1(c). Since the typical horizontal scale is three times larger than that of Case II, the time scale of the evolution considerably slows down compared with that of Case I and Case II as pointed out in the Appendix 1.B. We note that the flow pattern at $t=50$ is not so distorted from that at $t=0$. The elongation of cyclones also takes place (e.g., a weak event from $t=100$ to $t=125$ in the middle part, and a break-up event from $t=200$ to $t=225$ in the lower-right corner). However, the elongation is not so remarkable and the break-up is less frequent as compared with Case II. Instead, contours of cyclones are unstable and tend to oscillate considerably (near the lower-right part from $t=150$ to $t=200)$. In addition, cyclones are somewhat eager to merge, but merger is partial (near the lower-right part from $t=50$ to $t=100$, and in the upper-middle part from $t=225$ 
to $t=250$ ). The cyclone after merger is unstable and sometimes breaks up again (near the lower-right part from $t=100$ to $t=125$ ). In contrast, anticyclones are much more eager to merge and become larger scales. (See the events in the upper-left and upper-right parts throughout the evolution.)

\subsection{Asymmetry in statistical quantities}

In order to see asymmetric properties globally, some statistical quantities are examined as the spatial averages over the anticyclonic and cyclonic regions separately. We define the anticyclonic (cyclonic) region by the region with $\eta>0(\eta<0)$. This definition comes from our assumption that the sign of $\eta$ is essential for the asymmetry as implied by the nonlinear divergence in Eq. (1.1b). This definition also has another advantage. The velocity field is nearly in geostrophic balance, that is, the velocity vectors on the boundaries dividing the cyclonic region from the anticyclonic region are nearly parallel to contours with $\eta=0$. Thus the flux of a quantity across the boundaries will be small compared with the time rate of change of the quantity. In the following description, $\langle\cdots\rangle_{c}$ and $\langle\cdots\rangle_{a}$ denote the average over the cyclonic and anticyclonic regions, respectively. We hereafter show two contrasting cases, Case I and Case II.

Before describing the present analyses, we briefly review the evolution of a turbulent flow for the non-divergent case (e.g., Brachet et al. 1988; Santangelo et al. 1989). In decaying turbulence with a smooth initial distribution of vorticity, the flow evolution can be classified into three phases: (i) In the early phase, interactions between different scales develop. However, the smallest scales where dissipation works are not excited yet. Thus enstrophy dissipation remains small. In physical space, steep gradients of potential vorticity are formed between initial coherent 
vortices due to their differential advection. (ii) In the active phase of turbulence, all scales of motion are excited, and enstrophy dissipation increases till reaches its maximum value. In physical space, elongation of contours of potential vorticity frequently occurs. (iii) After the maximum enstrophy dissipation is attained, the dissipation exceeds the production of gradients of potential vorticity. Thus the evolution enters the decaying phase. In this later phase, enstrophy is mainly contained inside large-scale coherent vortices, where its dissipation is inactive except at merger. Thus enstrophy is gradually transferred to larger scales.

\subsubsection{Energy and enstrophy transfer}

First we examine the asymmetric nature in the energy and enstrophy transfer. The total energy density $E$ is the sum of the kinetic energy density $K$ plus the potential energy density $P$ as

$$
E=K+P
$$

where

$$
K=\frac{1}{2} h \boldsymbol{u} \cdot \boldsymbol{u}, \quad P=\frac{1}{2} F \eta^{2} .
$$

The potential enstrophy density $Q$ is defined as

$$
Q=\frac{1}{2} h q^{2}
$$

Of course, the spatial integrals of $E$ and $Q$ over the full domain are conserved in an inviscid case $(\nu=0)$ as can be seen from (1.10a), (1.10b)and (1.16). Since $K$ and $Q$ are cubic in the field variables, we introduce new variables as

$$
U=\sqrt{h} u, \quad V=\sqrt{h} v, \quad q^{\prime}=\sqrt{h} q-1,
$$

where the undisturbed value 1 is subtracted in the definition of $q^{\prime}$. The containing wavenumbers $k_{K}, k_{P}, k_{E}$ and $k_{Q}$ of kinetic energy, potential 
energy, total energy, and potential enstrophy are introduced, respectively as follows:

$$
\begin{aligned}
& k_{K^{*}}=\left[\frac{\int\left(|\nabla U|^{2}+|\nabla V|^{2}\right) d x d y}{\int\left(U^{2}+V^{2}\right) d x d y}\right]^{\frac{1}{2}}, \\
& k_{P}=\left[\frac{\int|\nabla \eta|^{2} d x d y}{\int \eta^{2} d x d y}\right)^{\frac{1}{2}}, \\
& k_{E}=\left[\frac{\int\left(|\nabla U|^{2}+|\nabla V|^{2}+F|\nabla \eta|^{2}\right) d x d y}{\int\left(U^{2}+V^{2}+F \eta^{2}\right) d x d y}\right]^{\frac{1}{2}}, \\
& k_{Q}=\left(\frac{\int\left|\nabla q^{\prime}\right|^{2} d x d y}{\int q^{\prime 2} d x d y}\right)^{\frac{1}{2}},
\end{aligned}
$$

where the integration is done over the cyclonic or anticyclonic region.

Figure 1.2 shows the evolution of $k_{E}$ for cyclones and anticyclones. For Case I, the energy of cyclones is slightly transferred to smaller scales, while that of anticyclones is slightly transferred to larger scales in the initial phase $t \lesssim 16$. In the active phase $16 \lesssim t \lesssim 33$, the energies of both cyclones and anticyclones are transferred to larger scales, which is a manifestation of the inverse cascade of energy. In the later phase $t \gtrsim 35$, however, the transfer rate for cyclones is reduced. For Case II, the energy transfer to smaller scales for cyclones is much more significant than that for Case $I$ in the initial phase $t \lesssim 15$ and continues by $t \sim 22$, which means the suppression of the inverse cascade of energy. In contrast, the energy of anticyclones is transferred to larger scales as in Case I. Note that the onset of the active phase around $t=16$ for Case I and around $t=15$ for Case II will be clear when seeing the evolution of the production rate of square $q$-gradients in Fig. 1.6 and that of the enstrophy dissipation rate in Fig. 1.7. 
Figure 1.3 shows the evolution of $k_{Q}$. For Case I, the enstrophy is transferred to smaller scales during the initial and active phases $t \lesssim 33$, which is a manifestation of the cascade of enstrophy. There are no significant differences between cyclones and anticyclones. Around $t=33, k_{Q}$ changes to decreasing, which demonstrates that the flow evolution enters the later phase of viscous decay. This time $t \sim 33$ well corresponds to the time of the maximum enstrophy dissipation as will be shown in Fig. 1.7(a). For Case II, the enstrophy is transferred to smaller scales during the initial and active phases $t \lesssim 32$ as in Case I. The difference of $k_{Q}$ between cyclones and anticyclones is not so remarkable as compared with that of $k_{E}$, although the enstrophy of cyclones is transferred slightly to smaller scales than that of anticyclones. This less discrepancy suggests that the effect of divergence is less significant at small scales where enstrophy is contained as pointed out by Farge and Sadourny (1989).

\subsubsection{Large-scale quantities}

In order to clarify the asymmetry in the energy transfer properties furthermore, we examine the energy exchange between kinetic and potential energy. The energy equations are

$$
\begin{aligned}
\frac{\partial K}{\partial t} & =-\Gamma_{K P}-D_{E}-\frac{1}{\varepsilon} \nabla \cdot\left[h \boldsymbol{u}\left(\varepsilon \frac{1}{2} \boldsymbol{u} \cdot \boldsymbol{u}+\eta\right)\right], \\
\frac{\partial P}{\partial t} & =\Gamma_{K P},
\end{aligned}
$$

where $\Gamma_{K P}$ is the energy conversion rate from kinetic to potential energy due to the stretching or contraction of fluid columns, and $D_{E}$ is the energy dissipation rate. These are expressed as

$$
\begin{aligned}
\Gamma_{K P} & =-\frac{1}{\varepsilon} \eta \nabla \cdot(h \boldsymbol{u})=-\frac{1}{\varepsilon}[\eta \nabla \cdot \boldsymbol{u}+\delta \eta \nabla \cdot(\eta \boldsymbol{u})], \\
D_{E} & =\nu \triangle(h \boldsymbol{u}) \cdot \triangle \boldsymbol{u} .
\end{aligned}
$$


The conversion rate $\Gamma_{K P}$ provides a good measure for the asymmetry, because the second term on the right-hand side of (1.11a) is asymmetric between cyclones and anticyclones. The contribution from this term cannot be neglected when the amplitude parameter $\delta$ is large enough. Since the containing scale of potential energy is larger than that of kinetic energy as will be shown in Fig. 1.4, $\left\langle\Gamma_{K^{P}}\right\rangle>0$ means the inverse cascade while $\left\langle\Gamma_{K^{-} P}\right\rangle<0$ means the suppression of the inverse cascade. Here we note that the cascade of energy will be inhibited as in the quasi-geostrophic case, when the velocity field is nearly composed of the rotational part (Farge and Sadourny 1989). In such cases, the energy and enstrophy conservation constrains nonlinear interactions as in the quasi-geostrophic case (Larichev and McWilliams 1991). That is, the backward transfer of potential energy and the forward transfer of kinetic energy occurs at scales larger than the radius of deformation. On the other hand, the backward transfer of kinetic energy and the forward transfer of squared vorticity occurs at scales smaller than the radius of deformation, since the stretching does not work well here. Thus the kinetic energy transferred from larger to smaller scales tends to pile up around scales comparable to the radius of deformation.

Figure 1.4 shows the evolution of $k_{K^{*}}$ and $k_{P}$ for cyclones and anticyclones, and Figure 1.5 shows the evolution of $\left\langle\Gamma_{K P}\right\rangle_{c}$ and $\left\langle\Gamma_{K P}\right\rangle_{a}$. For Case I, $\left\langle\Gamma_{K^{P} P}\right\rangle_{c}$ and $\left\langle\Gamma_{K P}\right\rangle_{a}$ are both positive during the initial and active phases $t \lesssim 34$, which means the persistence of the inverse cascade. Actually, $k_{K^{\prime}}$ shifts slightly toward larger wavenumbers but this shift stops around $k_{K^{\prime}}=4.8$, while $k_{P}$ decreases monotonically for both cyclones and anticyclones. During the duration $34 \lesssim t \lesssim 50$ in the later phase, $\left\langle\Gamma_{K^{P} P}\right\rangle_{C}$ is negative. Thus the inverse cascade is suppressed during this duration as can be seen from the facts that $k_{P}$ and $k_{E}$ for cyclones are nearly constant. Therefore the time average over $0 \leq t \leq 50$ of $\left\langle\Gamma_{K^{P} P}\right\rangle_{c}, 1.6 \times 10^{-4}$ is one-third 
smaller than that of $\left\langle\Gamma_{K P}\right\rangle_{a}, 4.5 \times 10^{-4}$. Although the vertical displacement is small, the above discrepancies between cyclones and anticyclones are a manifestation of the inherent asymmetry in the shallow-water equations. Such discrepancies are not observed in the quasi-geostrophic case (not shown). The energy dissipation rate is one order of magnitude smaller than the conversion rate. The maximum values of $\left\langle D_{E}\right\rangle_{c}$ and $\left\langle D_{E}\right\rangle_{a}$ are $2.1 \times 10^{-5}($ at $t=32.7)$ and $1.8 \times 10^{-5}$ (at $\left.t=30.2\right)$, respectively. Actually the total energy averaged over the full domain decreased to only $0.5 \%$ of its initial value by $t=50$.

For Case II, $\left\langle\Gamma_{K P}\right\rangle_{a}$ is positive almost throughout the evolution, which denotes the persistence of the inverse cascade as can be seen from the monotonic decrease of $k_{P}$ and $k_{E}$ for anticyclones. For cyclones, $\left\langle\Gamma_{L_{P} P}\right\rangle_{c}$ is negative by $t \sim 21$, which means the suppression of the inverse cascade. Actually, $k_{K}$ increases monotonically and $k_{P}$ is nearly constant by $t \sim 24$. Especially at the beginning of the early phase $(t \lesssim 4),\left\langle\Gamma_{K P}\right\rangle_{c}$ takes extremely large negative values, when the shrinking deformation of $q$ contours occurs as seen in Fig. 1.1(b). During $22 \lesssim t \lesssim 28,\left\langle\Gamma_{K P}\right\rangle_{c}$ takes positive large values, when the split cyclones (Event 1) coalesce again and the elongated cyclone (Event 2) rolls up. After $t \sim 30$ the flow evolution enters the decaying phase. In accordance with this, $\left\langle\Gamma_{K P}\right\rangle_{c}$ takes positive values, and $k_{P}$ and $k_{E}$ decrease monotonically. As a manifestation of the suppression of the inverse cascade, the time average of $\left\langle\Gamma_{K^{*} P}\right\rangle_{c}, 5.5 \times 10^{-5}$ is one order of magnitude smaller than that of $\left\langle\Gamma_{K P}\right\rangle_{a}, 4.4 \times 10^{-4}$. The maximum values of $\left\langle D_{E}\right\rangle_{c}$ and $\left\langle D_{E}\right\rangle_{a}$ are $3.6 \times 10^{-5}($ at $t=25.3)$ and $2.3 \times 10^{-5}$ (at $t=26.3$ ), respectively, which are one order of magnitude smaller than the time average of $\left\langle\Gamma_{K^{P} P}\right\rangle_{a}$ as in Case I. Actually the total energy decreased to only $0.7 \%$ of its initial value by $t=50$. This demonstrates that the total energy is approximately conserved in the case with large vertical displacements as in the quasi-geostrophic case and the case 
with small vertical displacements. This fact is quite contrast to the considerably irrotational cases investigated by Farge and Sadourny (1989).

\subsubsection{Small-scale quantities}

The activity of the elongation of contours of potential vorticity is measured by the magnitude $\mathcal{P}$ of gradients of potential vorticity defined as

$$
\mathcal{P}=\frac{1}{2} h|\nabla q|^{2}
$$

This quantity yields

$$
\frac{\partial \mathcal{P}}{\partial t}=\chi_{\mathcal{P}}-\nabla \cdot(\boldsymbol{u} \mathcal{P})-\varepsilon \nu h \nabla q \cdot \nabla\left(\frac{\triangle^{2} \zeta}{h}\right) .
$$

Here $\chi_{\mathcal{P}}$ represents the production rate of square $q$ gradients by nonlinear interactions. This term is defined as

$$
\chi_{\mathcal{P}}=-h \nabla q \cdot\left(\nabla \boldsymbol{u}^{\operatorname{tr}} \cdot \nabla q\right),
$$

where $\nabla \boldsymbol{u}^{\text {tr }}$ is the transposed tensor of the velocity gradient tensor $\nabla \boldsymbol{u}$ defined by

$$
\nabla \boldsymbol{u}^{\mathrm{tr}}=\left(\begin{array}{ll}
\frac{\partial u}{\partial x} & \frac{\partial v}{\partial x} \\
\frac{\partial u}{\partial y} & \frac{\partial v}{\partial y}
\end{array}\right)
$$

If $q$ contours tend to be statistically elongated, spatial average of $\chi_{\mathcal{P}}$ will be positive.

Figure 1.6 shows the evolution of $\left\langle\chi_{\mathcal{P}}\right\rangle_{c}$ and $\left\langle\chi_{\mathcal{P}}\right\rangle_{a}$. For Case I, the production rate increases intermittently after $t \sim 13$, which demonstrates that the flow evolution enters the active turbulent phase. In this case, the difference between $\left\langle\chi_{\mathcal{P}}\right\rangle_{c}$ and $\left\langle\chi_{\mathcal{P}}\right\rangle_{a}$ is not so remarkable. The time average over $0 \leq t \leq 50$ of $\left\langle\chi_{\mathcal{P}}\right\rangle_{c}, 1.5 \times 10^{-2}$ is nearly the same as that of $\left\langle\chi_{\mathcal{P}}\right\rangle_{a}, 1.3 \times 10^{-2}$. For Case II and Case II', $\left\langle\chi_{\mathcal{P}}\right\rangle_{c}$ is much larger than 
$\left\langle\chi_{\mathcal{P}}\right\rangle_{a}$. In the early phase $t<14,\left\langle\chi_{\mathcal{P}}\right\rangle_{c}$ is peaked around $t=2$, which corresponds to the distortion of cyclones to shrunk triangular shapes. After $t \sim 14,\left\langle\chi_{\mathcal{P}}\right\rangle_{c}$ increases intermittently, which demonstrates that the flow evolution enters the actively elongating phase. Three major peaks are observed around $t=18, t=24$ and $t=29$ which correspond to Event 1 , Event 2 and Event 3, respectively as seen in Fig. 1.1(b). The time average of $\left\langle\chi_{\mathcal{P}}\right\rangle_{c}, 5.3(2.7)$ is $4(3)$ times larger than that of $\left\langle\chi_{\mathcal{P}}\right\rangle_{a}, 1.3(0.87)$ for Case II (Case II'). The time average of $\left\langle\chi_{\mathcal{P}}\right\rangle_{c}$ for Case II is 350 times larger than that for Case I. The above facts come from the asymmetric structure of the $q$ gradient between cyclones and anticyclones when the amplitude of vertical displacements is large. Since the fluid depth of large-amplitude cyclones is much smaller than that of small-amplitude cyclones and arbitrary-amplitude anticyclones, $\mathcal{P}$ of the former case is much larger than that of the latter cases. Thus the production rate of $\mathcal{P}$ for the former case becomes much larger than that for the latter cases when the elongation occurs by some mechanism.

The active elongation of $q$ contours accompanies active enstrophy dissipation. The potential enstrophy $Q$ per unit area yields

$$
\frac{\partial Q}{\partial t}=-\varepsilon D_{Q}-\nabla \cdot(\boldsymbol{u} Q)
$$

where $D_{Q}$ is the enstrophy dissipation rate per unit area defined by

$$
D_{Q}=\nu \triangle q \cdot \triangle \zeta
$$

Figure 1.7 shows the evolution of $\left\langle D_{Q}\right\rangle_{c}$ and $\left\langle D_{Q}\right\rangle_{a}$. For Case I, the evolution of $\left\langle D_{Q}\right\rangle_{c}$ and $\left\langle D_{Q}\right\rangle_{a}$ quite resembles that of $\left\langle\chi_{\mathcal{P}}\right\rangle_{c}$ and $\left\langle\chi_{\mathcal{P}}\right\rangle_{a}$, since the production of small-scale $q$ gradients, which is associated with the generation of thin filaments, causes active enstrophy dissipation. Actually each peak of the sequences $\left\langle D_{Q}\right\rangle_{c}$ and $\left\langle D_{Q}\right\rangle_{a}$ well follows each peak of the sequences $\left\langle\chi_{\mathcal{P}}\right\rangle_{c}$ and $\left\langle\chi_{\mathcal{P}}\right\rangle_{a}$. The dissipation rates $\left\langle D_{Q}\right\rangle_{c}$ and $\left\langle D_{Q}\right\rangle_{a}$ 
reach the maximum values around $t=33$ and $t=31$, respectively, each of which well corresponds to the time when $k_{Q}$ begins to decrease as seen in Fig. 1.3. The time average over $0 \leq t \leq 50$ of $\left\langle D_{Q}\right\rangle_{c}, 2.6 \times 10^{-4}$ is nearly the same as that of $\left\langle D_{Q}\right\rangle_{a}, 2.3 \times 10^{-4}$. For Case II, $\left\langle D_{Q}\right\rangle_{c}$ in the early phase remains small, although the strong production of $q$ gradients takes place around $t=2$. This is because the distortion of $q$ contours in the early phase does not exhibit the formation of filaments. The enstrophy dissipation increases intermittently in the active phase after $t \sim 14$. The three major peaks also follow those in the sequence of $\left\langle\chi_{\mathcal{P}}\right\rangle_{c}$. The dissipation rates $\left\langle D_{Q}\right\rangle_{c}$ and $\left\langle D_{Q}\right\rangle_{a}$ reach the maximum values around $t=32$ and $t=26$, respectively, each of which well corresponds to the time when $k_{Q}$ begins to decrease as in Case I. The time average of $\left\langle D_{Q}\right\rangle_{c}$, $9.5 \times 10^{-3}\left(6.0 \times 10^{-3}\right)$ is also $4(3)$ times larger than that of $\left\langle D_{Q}\right\rangle_{a}, 2.3 \times 10^{-3}$ $\left(1.8 \times 10^{-3}\right)$ for Case II (Case II'). The enstrophy averaged over the full domain decreased to $8.5 \%(5.4 \%)$ of its initial value by $t=50$. The time average of $\left\langle D_{Q}\right\rangle_{c}$ for Case II is 16 times larger than that for Case I. The above results demonstrate that the active elongation of cyclones much intensifies the enstrophy transfer to smaller scales (enstrophy cascade) when the vertical displacement is large.

\subsection{Elongation of contours of potential vorticity}

The elongation of $q$ contours is a manner of the cascade of enstrophy in physical space. For vortices with small amplitude of vertical displacements or anticyclones with arbitrary amplitude, this accompanies the inverse cascade of energy, while for cyclones with large amplitude, this accompanies the suppression of the inverse cascade of energy. The above facts suggest that a mechanism for the elongation of large-amplitude cyclones will be different from that of small-amplitude vortices or arbitraryamplitude anticyclones. 
An alternative measure for the activity of the elongation of $q$ contours is the production rate of $q$ gradients. The elongation is essentially caused by an inviscid mechanism (Sulem et al. 1983; Melander et al. 1987), hence we treat an inviscid equation here. The gradient of potential vorticity yields

$$
\frac{D}{D t} \nabla q=-\nabla \boldsymbol{u}^{\mathrm{tr}} \cdot \nabla q
$$

where $\nabla \boldsymbol{u}^{\text {tr }}$ is defined by (1.15). Here we rewrite the components of $\nabla \boldsymbol{u}^{\text {tr }}$ in terms of strain and vorticity as

$$
\nabla \boldsymbol{u}^{\mathrm{tr}}=\left(\begin{array}{cc}
s_{11} & s_{12}+\frac{1}{2} \zeta \\
s_{12}-\frac{1}{2} \zeta & s_{22}
\end{array}\right) .
$$

According to Weiss (1991), we assume a temporal and spatial scale separation between velocity and velocity gradients. In other words, strain and vorticity are assumed to be frozen as far as the dynamics of $q$ gradients is concerned. This assumption appears reasonable since the spatial scale of a $q$ gradient is smaller than that of strain and vorticity, and this will imply that the former changes faster than the latter in time. Under this assumption, (1.18) is linear in a Lagrangian frame, thus is assumed to have solutions of the form $\nabla q \propto e^{\lambda_{ \pm} t}$. Here

$$
\lambda_{ \pm}=-s_{d} \pm \sqrt{\Lambda}
$$

where

$$
\begin{aligned}
& s_{d}=\frac{1}{2}\left(s_{11}+s_{22}\right), \\
& \Lambda=\frac{1}{4}\left(s_{11}-s_{22}\right)^{2}+s_{12}^{2}-\frac{1}{4} \zeta^{2} .
\end{aligned}
$$

Since the present initial conditions are in gradient-wind balance, we can assume $\left|s_{d}\right|<\sqrt{|\Lambda|}$ at all points and at all times. Then the behavior of $q$ 
gradients is approximately determined by the sign of $\Lambda$, that is, by the relative magnitude of strain and vorticity. In the regions with $\Lambda>0$, $\lambda_{+}(>0)$ and $\lambda_{-}(<0)$ are real. Thus the motion of $q$-gradients is said to be hyperbolic. The $q$-gradients will tend to grow in the direction of the eigenvector associated with $\lambda_{+}$. On the other hand, in the regions with $\Lambda<0, \lambda_{ \pm}$are approximately pure imaginary if $\left|s_{d}\right| \ll \sqrt{-\Lambda}$. Thus the motion of $q$-gradients is said to be elliptic, that is, the $q$-gradients will behave in an oscillatory manner. Inside the vortex core where $\Lambda<0, q$ gradients will not tend to grow, thus enstrophy dissipation will be inactive. In contrast, outside the vortex core where $\Lambda>0, q$ gradients will tend to grow which leads to active enstrophy dissipation. The validity of Weiss' assumption has been confirmed in the non-divergent quasi-geostrophic case (Weiss 1991; McWilliams 1984; Brachet et al. 1988).

In order to examine the applicability of the above assumption to the present shallow-water cases, we show the evolution of $q, \Lambda$ and $\mathcal{P}$. Figure 1.8 shows a typical case of two cyclones just before merging for Case I. Here we introduce an appropriate $q$ contour inside the core region $\Lambda<0$. The cyclone located in the upper-left corner of the panel at $t=28$ is going to merge with the cyclone located at the center, and is considerably distorted as approaching the latter cyclone. Note that the square $q$ gradients $\mathcal{P}$ are intensified not inside the core but around the periphery of the core of the former cyclone. The square $q$ gradients are also intensified along the boundaries between cyclones and anticyclones, since $\zeta \sim 0$, i.e., $\Lambda>0$ in the neighborhood of these boundaries. These observations are quite consistent with the above assumption. Next we show a typical case (Event 1) of an elongating cyclone for Case II in Fig. 1.9. The cyclone located at the center of the panel is in the strain field by two anticyclones located on its lower and upper-left sides, and is elongated in the leftward and lower-rightward directions where $\Lambda$ is positive. A surprising feature is 
that square $q$ gradients are considerably intensified just inside the vortex core where $\Lambda$ is negative. The invalidity of the above assumption comes from the break-down of the assumption of the temporal scale separation. Namely, the magnitudes of strain and vorticity increase inside the vortex core as $\mathcal{P}$ increases. The above facts suggest that a mechanism for the elongation of large-amplitude cyclones will be associated with some type of instability, and is not associated with the straining effect by surrounding vortices in contrast with that of small-amplitude cyclones and arbitrary-amplitude anticyclones. Finally, we note the considerable difference of the values of $\mathcal{P}$ between these two cases as pointed out in $\S 1.4 .3$.

\subsection{Summary and discussion}

The free evolution of many interacting vortices is investigated numerically in a rotating shallow-water system. When the amplitude of vertical displacements is considerably large and the horizontal scale is comparable to the radius of deformation, a remarkable asymmetric evolution is observed between cyclones and anticyclones. Equi-contours of potential vorticity $q$ of cyclones are frequently elongated, and as a result a single vortex breaks up into two vortices. Thus the merger of cyclones is suppressed. In contrast, anticyclones frequently merge as in the quasi-geostrophic case. The elongation of large-amplitude cyclones accompanies the stretching of fluid columns associated with the energy conversion from potential to kinetic energy. Since the stretching does not work at scales much smaller than the radius of deformation, the elongation does not cause the cascade of energy, but causes the suppression of the inverse cascade of energy. The initial magnitude of $q$ gradients is considerably large for large-amplitude cyclones. Thus the elongation simultaneously accompanies the strong production of $q$ gradients which leads to active enstrophy cascading. 
Weiss' hypothesis is applied to the elongation of large-amplitude cyclones. But as opposed to the expectation, it is found that $q$ gradients are intensified just inside the vortex core where vorticity dominates strain. This is because strain and vorticity increase inside the core as the core elongates. Thus the assumption of the temporal scale separation of strain/vorticity and $q$ gradients becomes invalid. This fact suggests that the mechanism for the elongation of large-amplitude cyclones will be associated with some kind of instability, and is not associated with the straining effect by surrounding vortices in contrast with the quasigeostrophic cases. In order to clarify the mechanism for the elongation of large-amplitude cyclones, the evolution and stability of an elliptical vortex will be investigated in the next part of the present work.

When the horizontal scale is a few times larger than the radius of deformation, that is, in the frontal geostrophic regime, the elongation of $q$ contours is not so remarkable and the break-up of cyclones is less frequent as compared with the case comparable to the radius of deformation. This fact suggests that the cubic nonlinearity alone is not responsible for the remarkable asymmetry between cyclones and anticyclones. Finally, it is notable that a large-amplitude cyclone tends to break up into two vortices but never breaks up into many smaller vortices. This fact is consistent with the expectation on the basis of the enstrophy conservation described in $\S 1.1$, and is quite contrast to the results by Cushman-Roisin and Tang (1990).

\section{Appendix 1.A. Balance equations}

In Eq. (1.4), the irrotational component $\nabla \phi$ is assumed to be $O(\varepsilon)$ smaller than the rotational component $\hat{z} \times \nabla \psi$. In this case with neglecting the viscosity, the shallow-water equations (1.5a), (1.5b) and (1.1b) 
are reduced to the equations correct to $O(\varepsilon)$ as follows:

$$
\begin{aligned}
& \varepsilon\left[\frac{\partial \zeta}{\partial t}+J(\psi, \zeta)\right]+d=0 \\
& \triangle \eta=\zeta-2 \varepsilon \Lambda_{r}, \\
& \varepsilon F\left[\frac{\partial \eta}{\partial t}+J(\psi, \eta)\right]+d=0
\end{aligned}
$$

respectively, where

$$
\Lambda_{r}=\left(\frac{\partial^{2} \psi}{\partial x \partial y}\right)^{2}-\frac{\partial^{2} \psi}{\partial x^{2}} \frac{\partial^{2} \psi}{\partial y^{2}} .
$$

By taking the Laplacian of (1.A1c), and subtracting $F$ times Eq. (1.A1a) from this,

$$
\varepsilon F \frac{\partial}{\partial t}(\triangle \eta-\zeta)+\varepsilon F[\triangle J(\psi, \eta)-J(\psi, \zeta)]+(\triangle-F) d=0
$$

is obtained. By differentiating $\varepsilon F$ times Eq. (1.A1b) with respect to time, and subtracting Eq. (1.A3) from this,

$$
(\triangle-F) d=\varepsilon F[J(\psi, \zeta)-\triangle J(\psi, \eta)]+2 \varepsilon^{2} F \frac{\partial \Lambda_{r}}{\partial t}
$$

is obtained. The set of the equations (1.A1a), (1.A1b) and (1.A4) are called the balance equations. The original shallow-water equations require three field variables, while the balance equations require only one variable $\psi$. In the balance equations, the surface elevation $\eta$ is first determined from (1.A1b) and the velocity potential $\phi$ is then determined from the combination of (1.A1a) and (1.A4).

\section{Appendix 1.B. Various geostrophic regimes}

When the Rossby number $\varepsilon$ is small (i.e., $\varepsilon \ll 1$ ), fluid motions governed by the rotating shallow-water equations fall in one of various geostrophic regimes (Williams and Yamagata 1984). Here we treat inviscid 
equations for brevity. We expand the velocity $\boldsymbol{u}$ and the surface elevation $\eta$ in powers of $\varepsilon$ as

$$
\boldsymbol{u}=\boldsymbol{u}^{(0)}+\varepsilon \boldsymbol{u}^{(1)}+\cdots, \quad \eta=\psi+\varepsilon \eta^{(1)}+\cdots .
$$

At the lowest order of (1.1a), geostrophic balance is satisfied as

$$
\boldsymbol{u}^{(0)}=\hat{z} \times \nabla \psi \text {. }
$$

At the next order, $O(\varepsilon)$ of $(1.1 \mathrm{a})$, the ageostrophic velocity $\boldsymbol{u}^{(1)}$ yields

$$
\boldsymbol{u}^{(1)}=\hat{z} \times\left[\nabla \eta^{(1)}+\partial_{t} \boldsymbol{u}^{(0)}+\left(\boldsymbol{u}^{(0)} \cdot \nabla\right) \boldsymbol{u}^{(0)}\right]
$$

and its horizontal divergence is

$$
\nabla \cdot \boldsymbol{u}^{(1)}=-\partial_{t}(\triangle \psi)-J(\psi, \triangle \psi)
$$

Under the above assumption, the original shallow-water equations are reduced to one of geostrophic equations depending on $F$. The following derivations on an $f$-plane are somewhat different from derivations on a $\beta$-plane by the following previous works.

\section{a. Quasi-geostrophic regime}

We assume that the horizontal scale of motion is assumed to be comparable to the radius of deformation (i.e., $F \sim O(1)$ ). In this case, the amplitude parameter $\delta=\varepsilon F$ is small (i.e., $\delta \sim \varepsilon \ll 1$ ), that is, vertical displacements are small compared with fluid depth. Thus the nonlinear divergence does not appear up to $O(\varepsilon)$. Then the continuity equation $(1.1 \mathrm{~b})$ is reduced to the quasi-geostrophic equation,

$$
\partial_{t}(\triangle \psi-F \psi)+J(\psi, \triangle \psi)=0 \text {. }
$$

(e.g., Williams and Yamagata 1984; Pedlosky 1987) 
b. Frontal geostrophic regime

We assume that the horizontal scale is a few times larger than the radius of deformation as $F^{-1} \sim \varepsilon \ll 1$. A new feature of this case is that vertical displacements are comparable to fluid depth (i.e., $\delta=\varepsilon F \sim O(1)$ ). Thus all terms in (1.1b) become important up to $O(\varepsilon)$ as

$$
\varepsilon F \partial_{t} \psi+\varepsilon \nabla \cdot \boldsymbol{u}^{(1)}+\varepsilon^{2} F \nabla \cdot\left(\psi \boldsymbol{u}^{(1)}+\eta^{(1)} \boldsymbol{u}^{(0)}\right)=0 .
$$

Since all terms except the time derivative term in (1.B5) are $O(\varepsilon)$, the time scale of an evolution should be $O(\varepsilon)$ smaller than that in the quasigeostrophic regime. Hence we introduce a slow time coordinate $T$ as $\partial_{t} \rightarrow \varepsilon \partial_{T}$. The term $\partial_{t}(\Delta \psi)$ in (1.B3) is $O(\varepsilon)$ smaller than other terms, and the nonlinear divergence is

$$
\nabla \cdot\left(\psi \boldsymbol{u}^{(1)}+\eta^{(1)} \boldsymbol{u}^{(0)}\right)=-J\left(\psi, \psi \Delta \psi+\frac{1}{2}|\nabla \psi|^{2}\right) .
$$

Thus Eq. (1.B5) is reduced to

$$
\delta \partial_{T} \psi-J(\psi, \triangle \psi)-\delta J\left(\psi, \psi \Delta \psi+\frac{1}{2}|\nabla \psi|^{2}\right)=0,
$$

which is termed the frontal geostrophic equation by Cushman-Roisin (1986), while is termed $\mathrm{PG}_{2}$ by Williams and Yamagata (1984). The last Jacobian term, which is called a cubic nonlinearity, represents the effect of finite vertical displacements, and breaks the symmetry under the transformation $\psi \rightarrow-\psi$ and $(x, y) \rightarrow(x,-y)$ as opposed to the quasigeostrophic equation. 

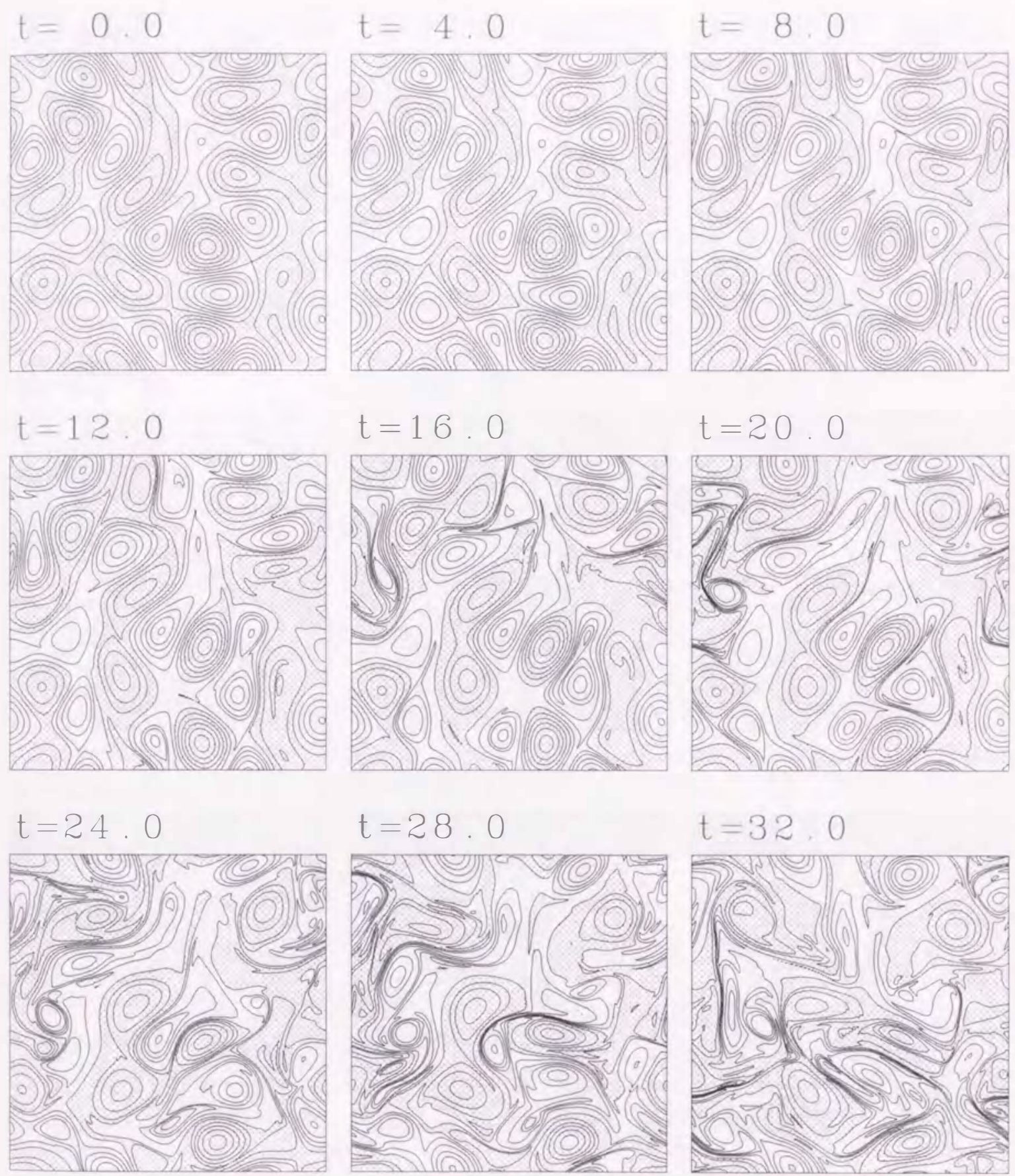

Fig. 1.1(a). Evolution of the potential vorticity $q$ for Case I. The anticyclonic regions $(q<1)$ are shaded. The contour interval is 0.03 . 

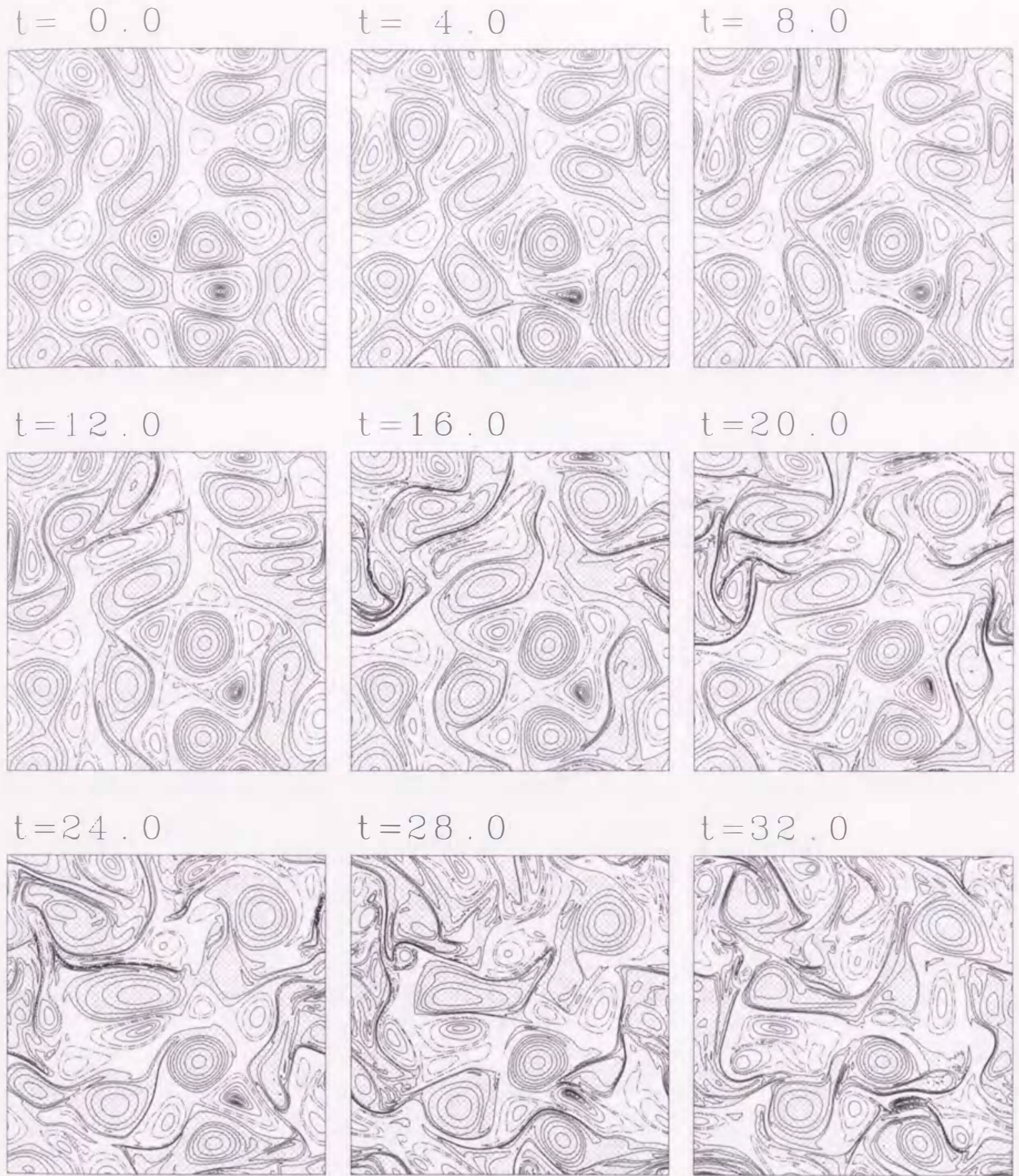

Fig. 1.1(b). Same as Fig. 1.1(a) but for Case II. The interval of solid contours is 2.5 in the non-shaded regions, and is 0.15 in the shaded regions. The interval of dash-dotted contours is 0.5 , which is used to show the relatively weak cyclonic flows. 

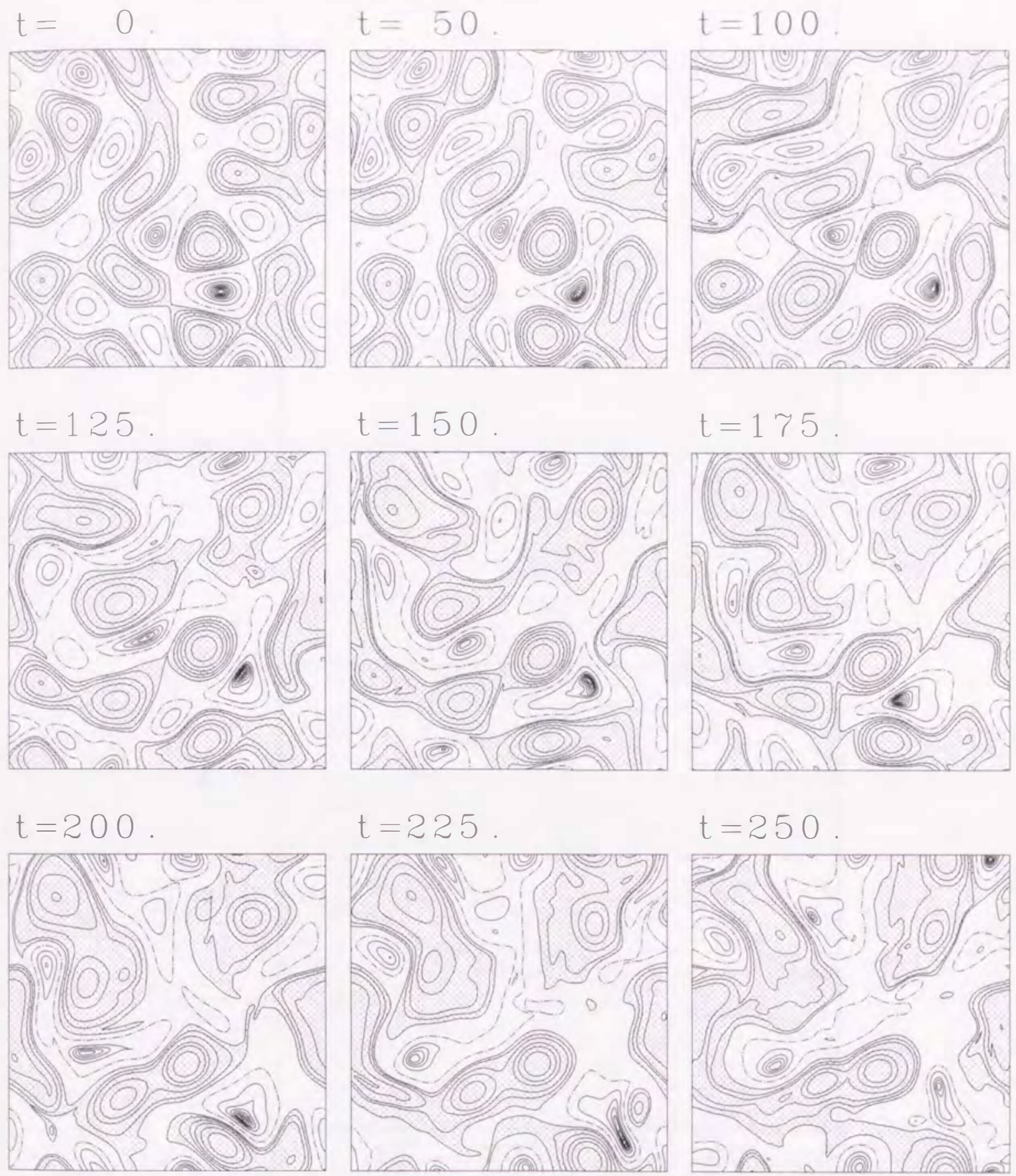

Fig. 1.1(c). Same as Fig. 1.1(b) but for Case III. The interval of solid contours is 1.5 in the non-shaded regions, and is 0.1 in the shaded regions. The dash-dotted contours are with $q=1.5$. 


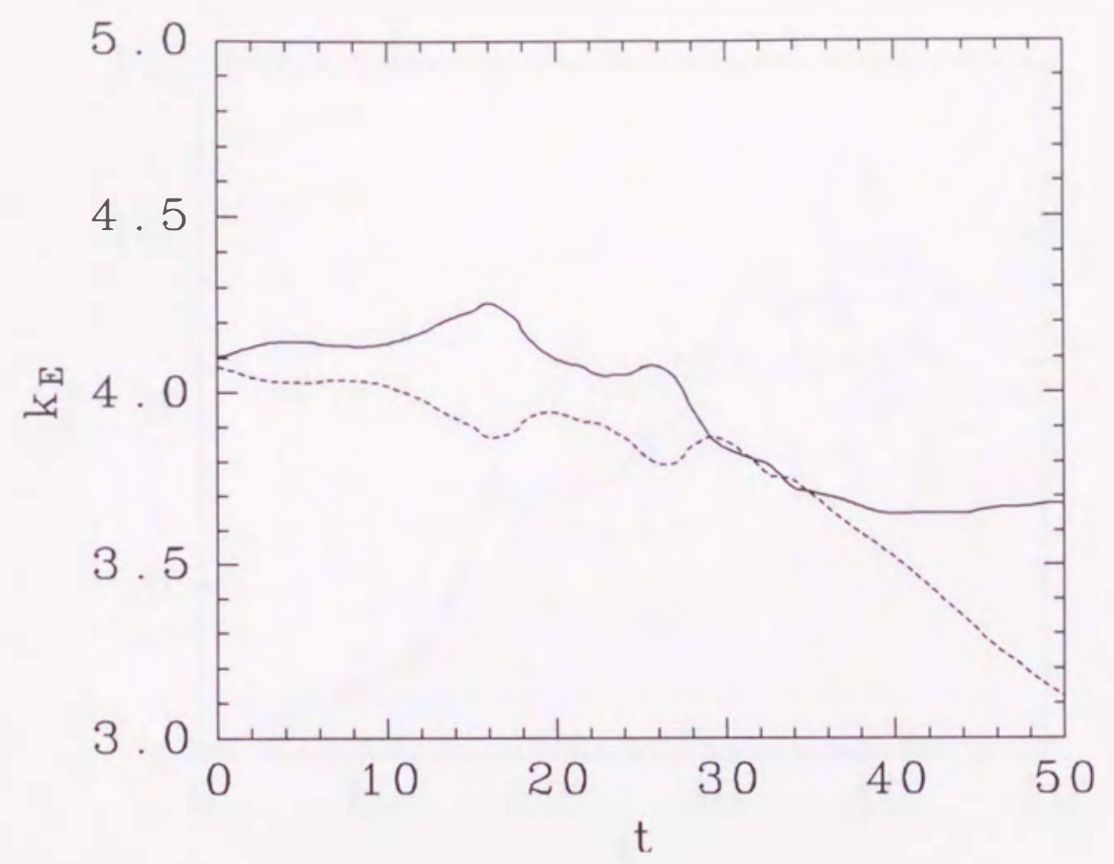

(a)

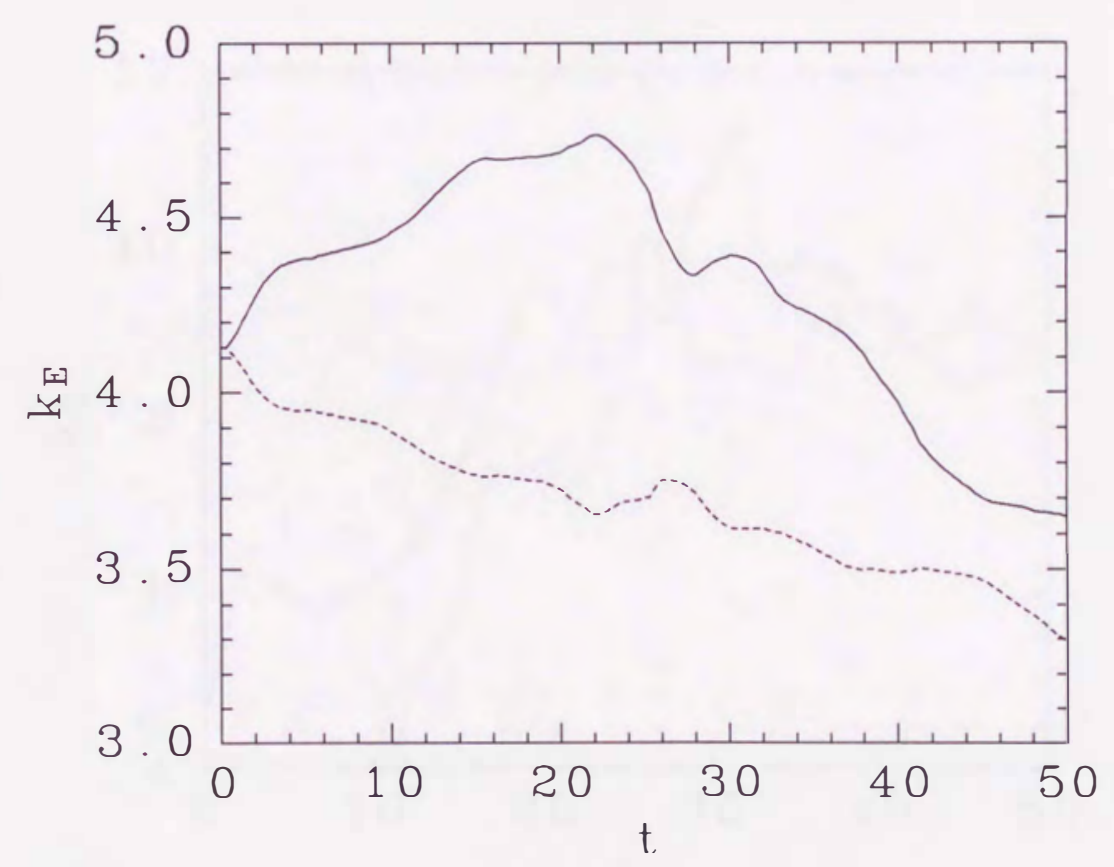

(b)

Fig. 1.2. Evolution of the containing wavenumber $k_{E}$ of total energy of cyclones (solid line) and anticyclones (dashed line): (a) for Case I and (b) for Case II. 


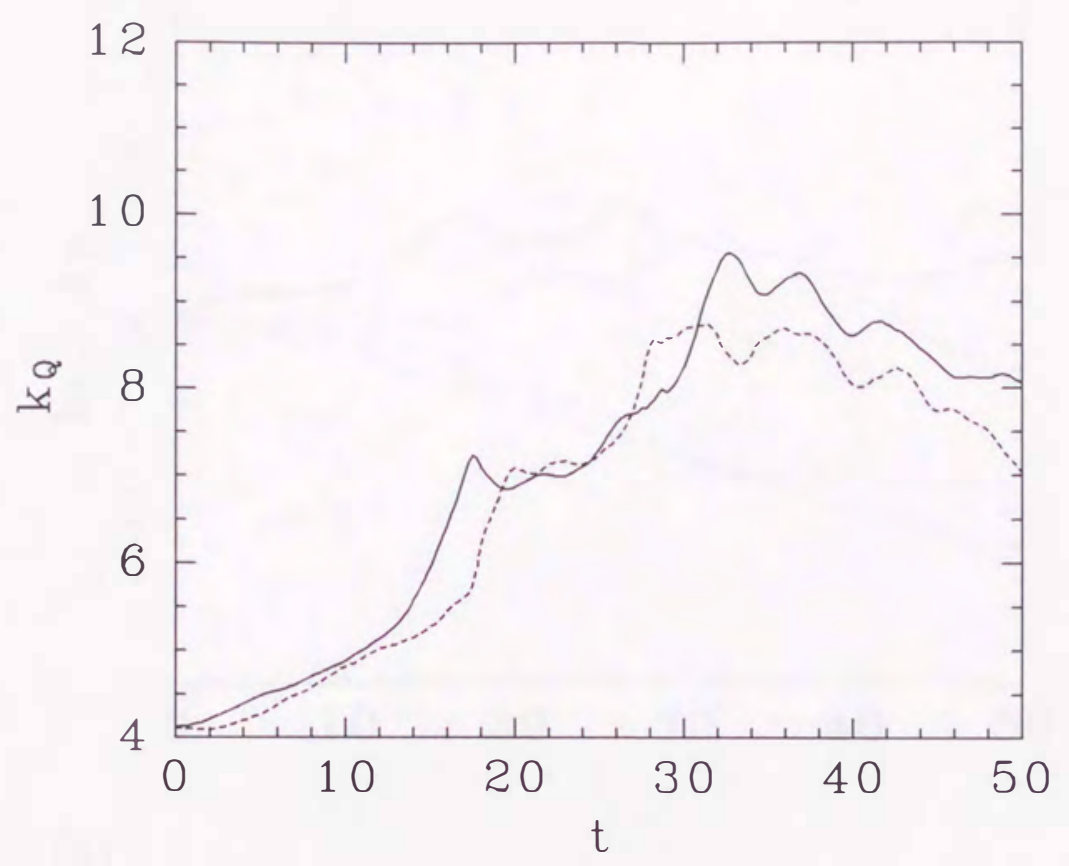

(a)

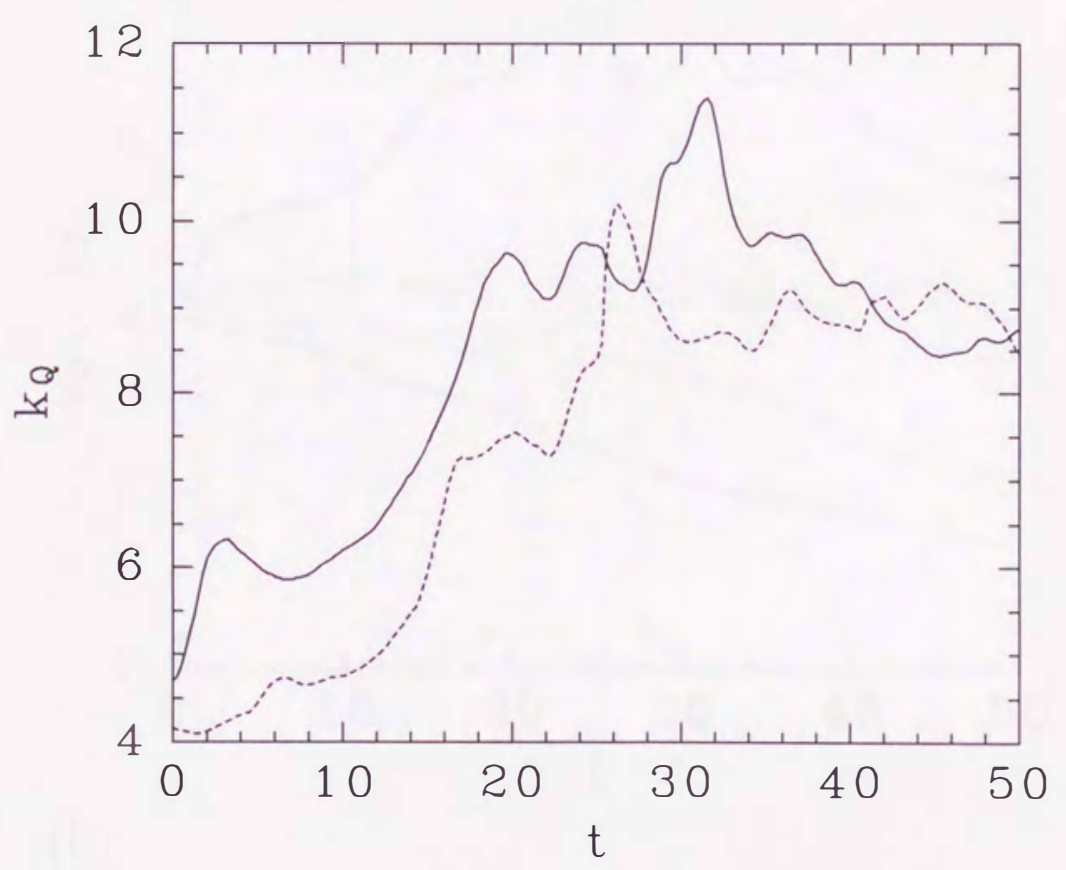

(b)

Fig. 1.3. Same as Fig. 1.2 but the evolution of the containing wavenumber $k_{Q}$ of potential enstrophy. 


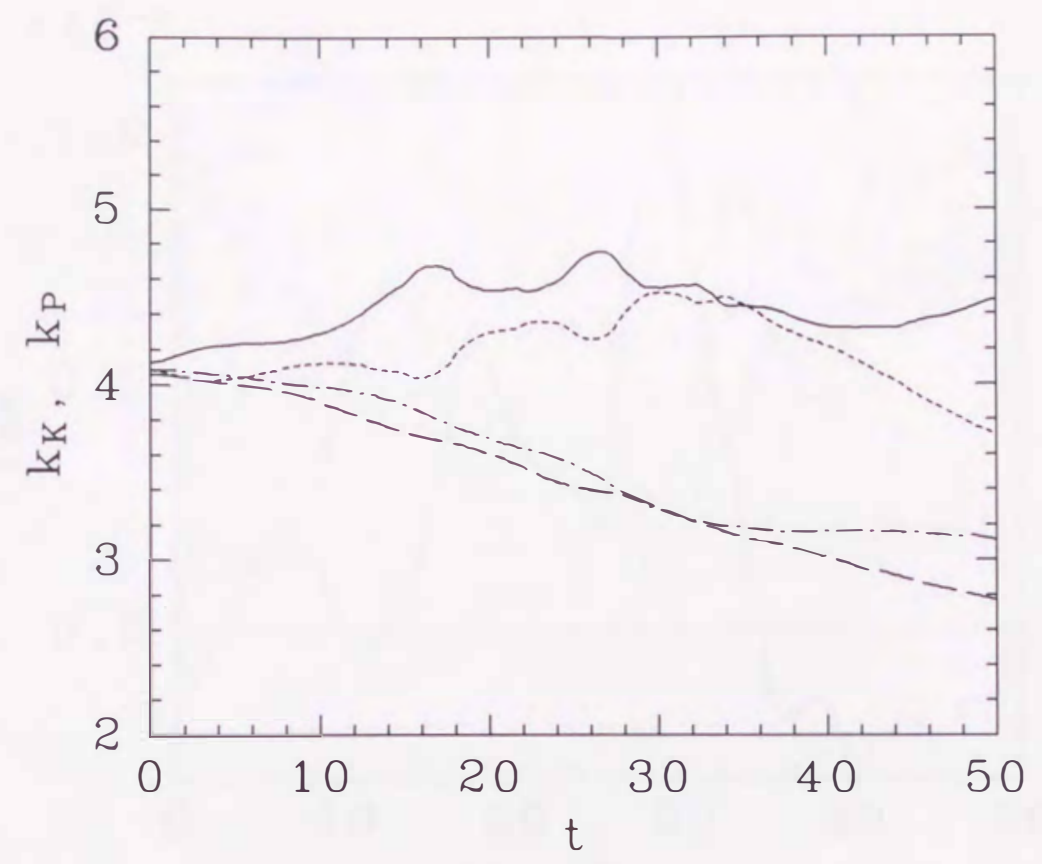

(a)

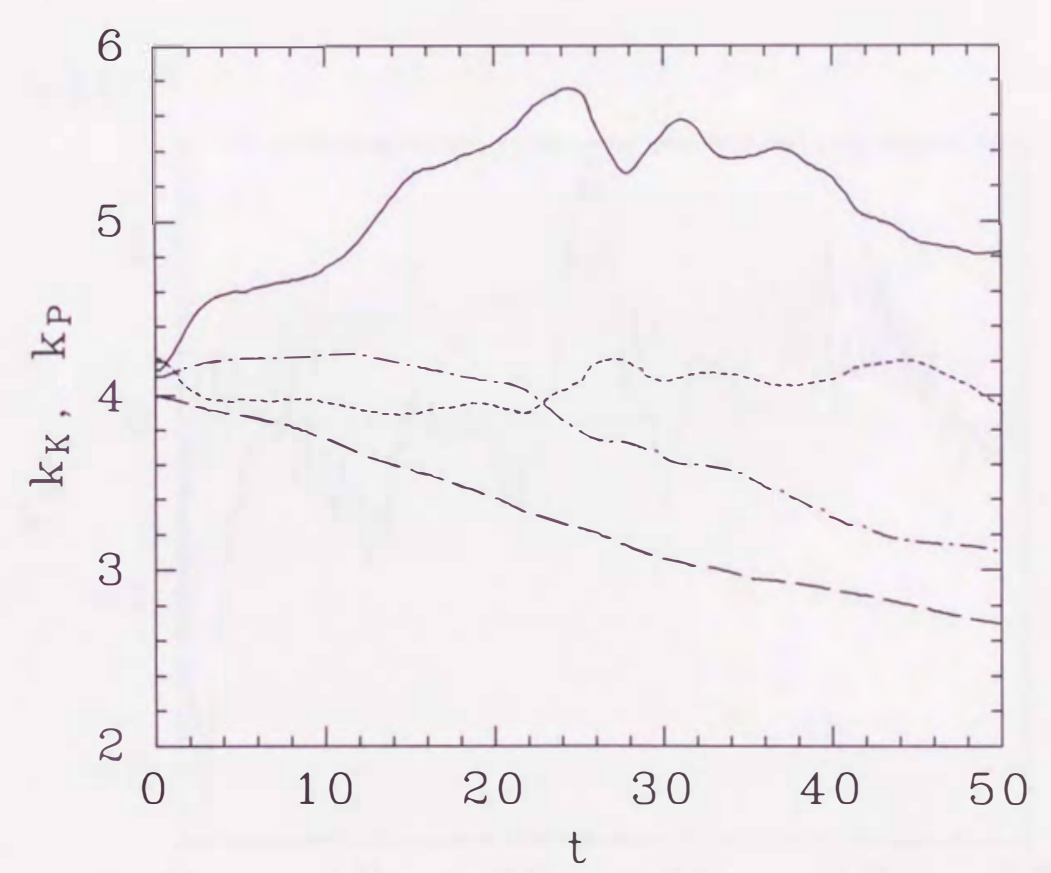

(b)

Fig. 1.4. Evolution of the containing wavenumbers of kinetic energy of cyclones (solid line), kinetic energy of anticyclones (dotted line), potential energy of cyclones (dash-dotted line) and potential energy of anticyclones (dashed line): (a) for Case I and (b) for Case II. 


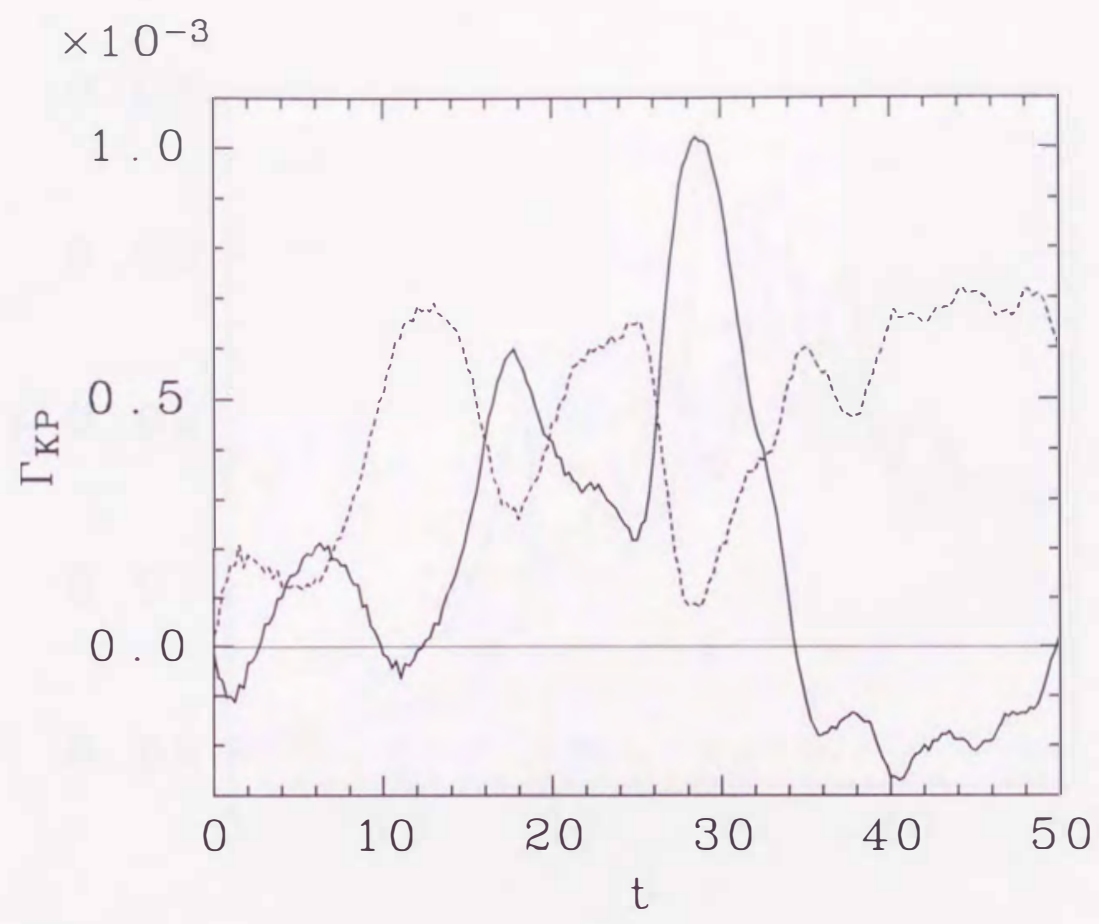

(a)

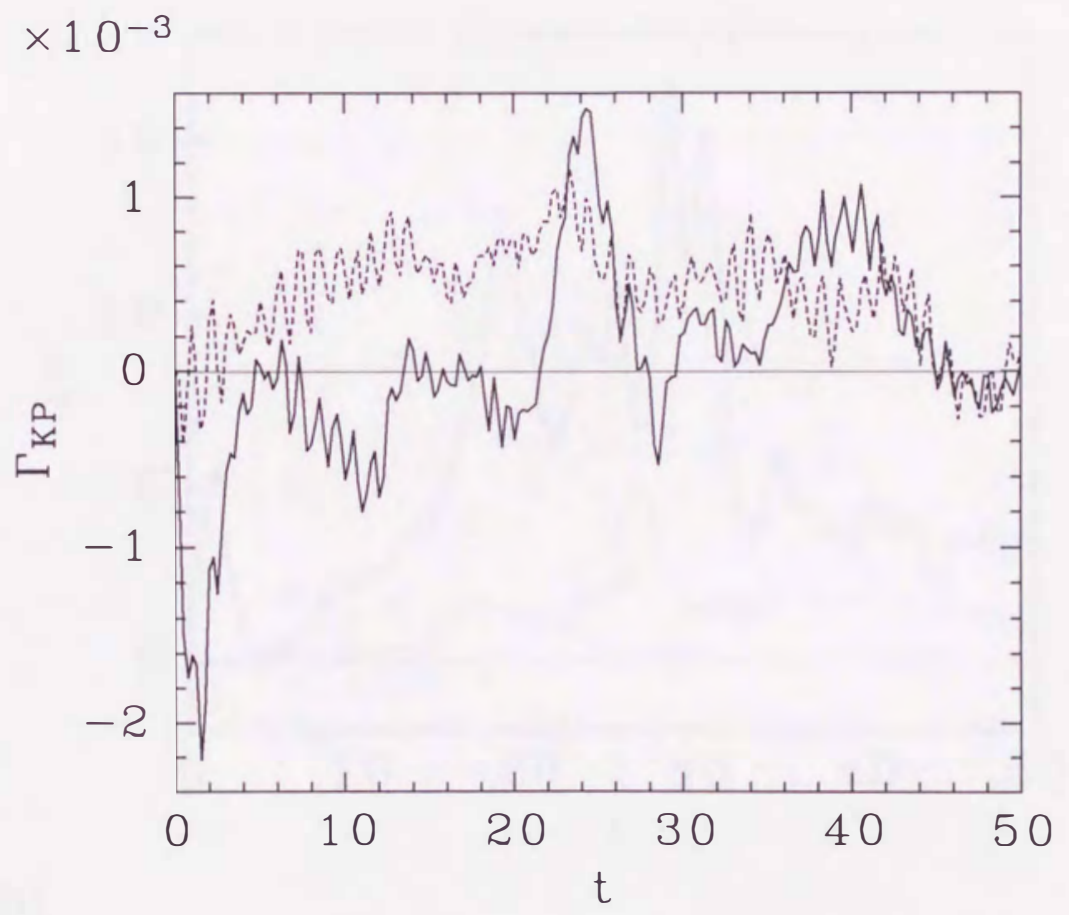

(b)

Fig. 1.5. Evolution of the conversion rate from kinetic to potential energy of cyclones (solid line) and anticyclones (dashed line): (a) for Case I and (b) for Case II. 


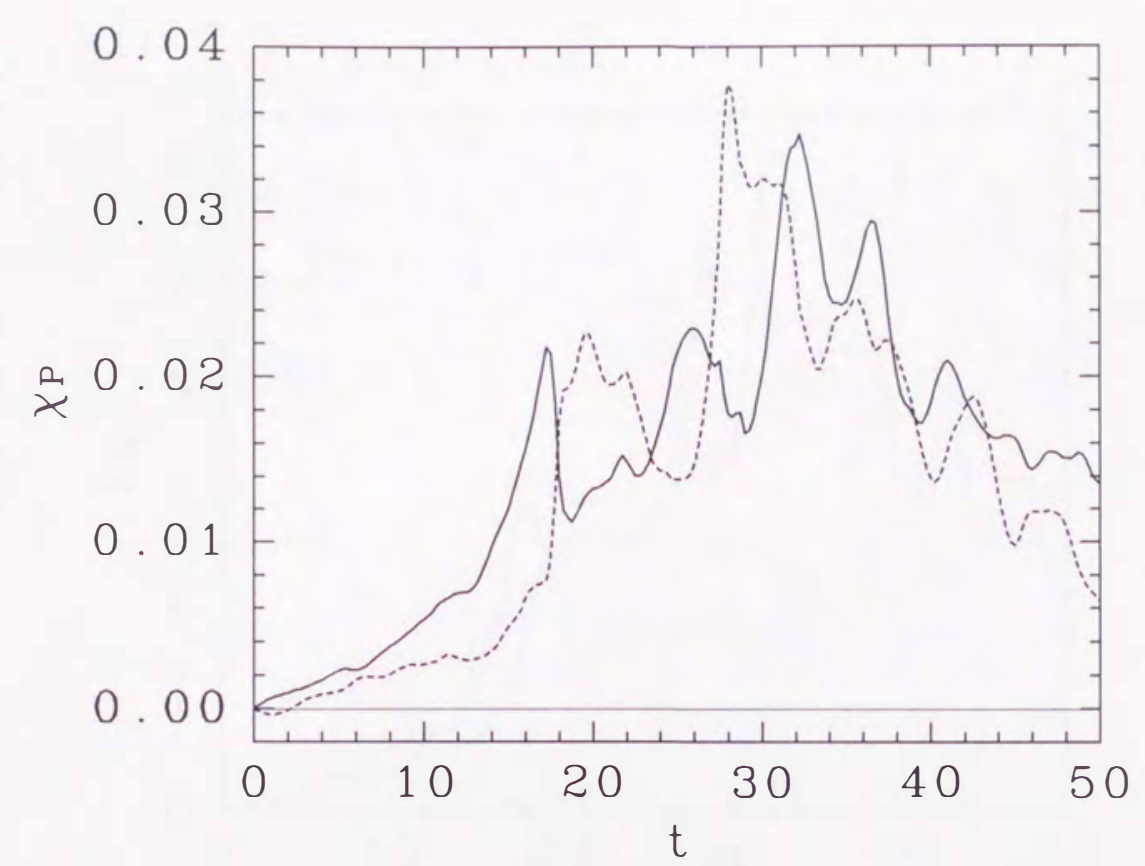

(a)

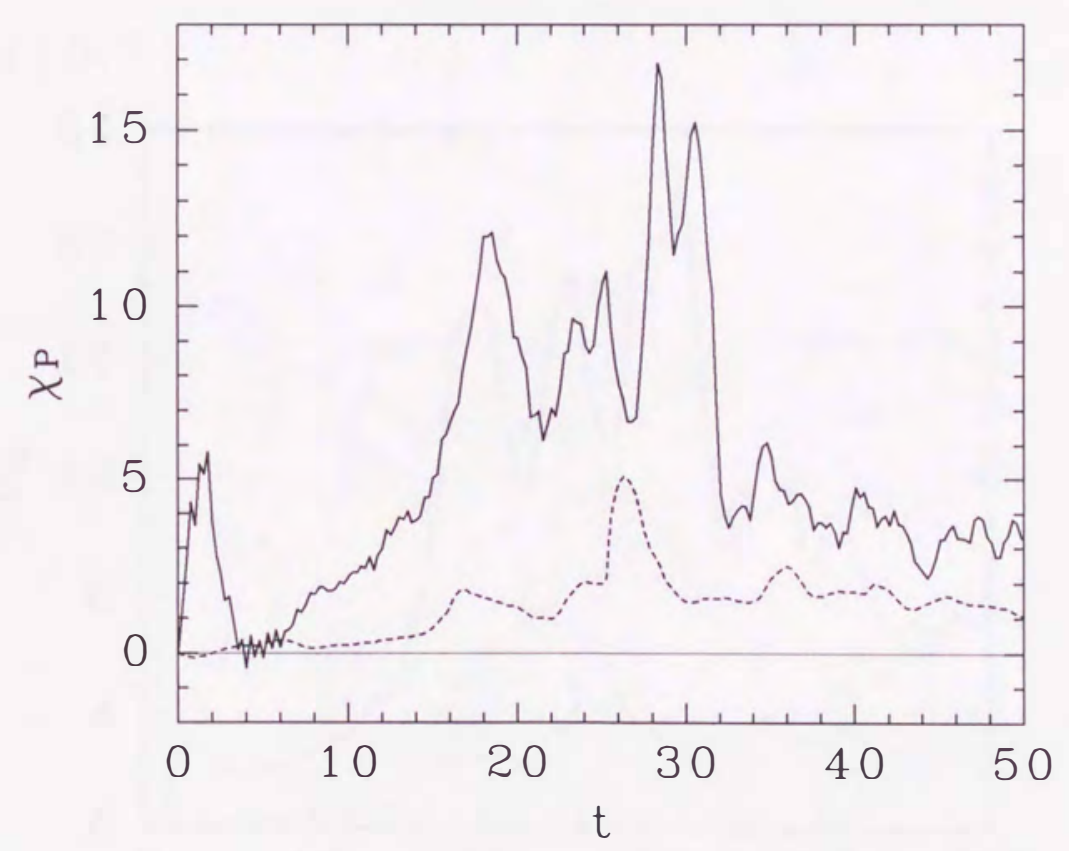

(b)

Fig. 1.6. Evolution of the production rate of square $q$ gradients of cyclones (solid line) and anticyclones (dashed line): (a) for Case I and (b) for Case II. 
$\times 10^{-4}$

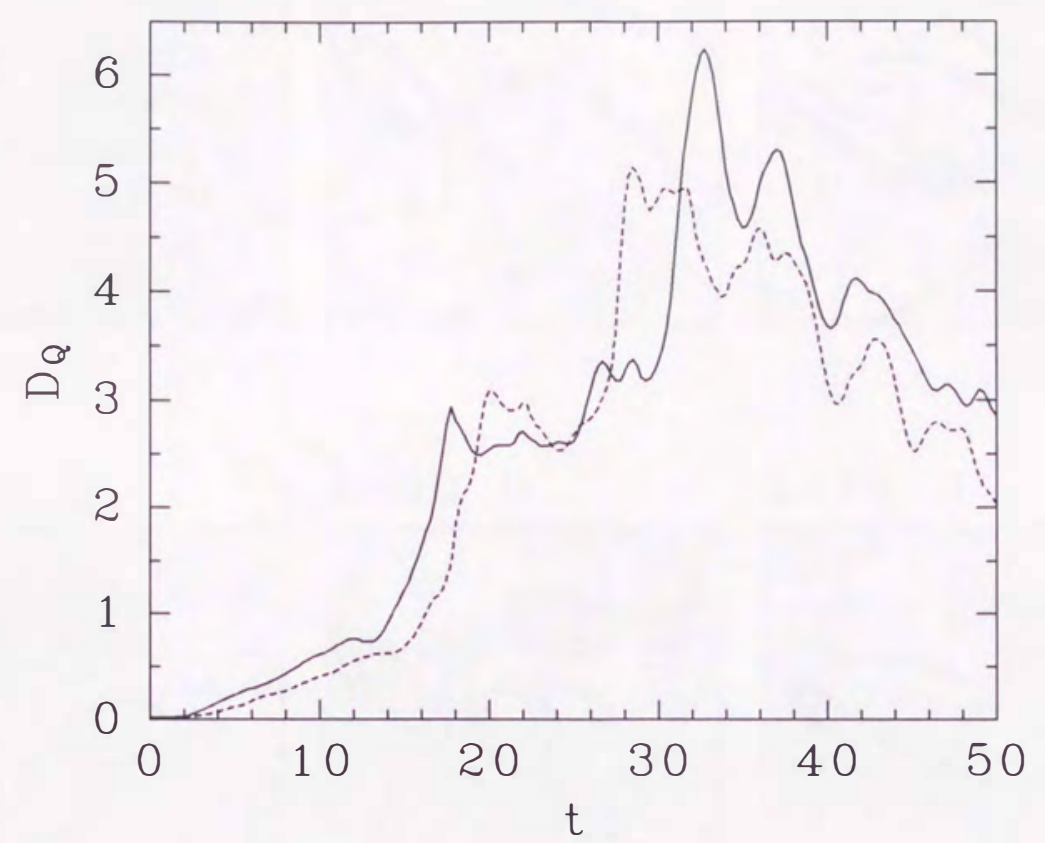

(a)

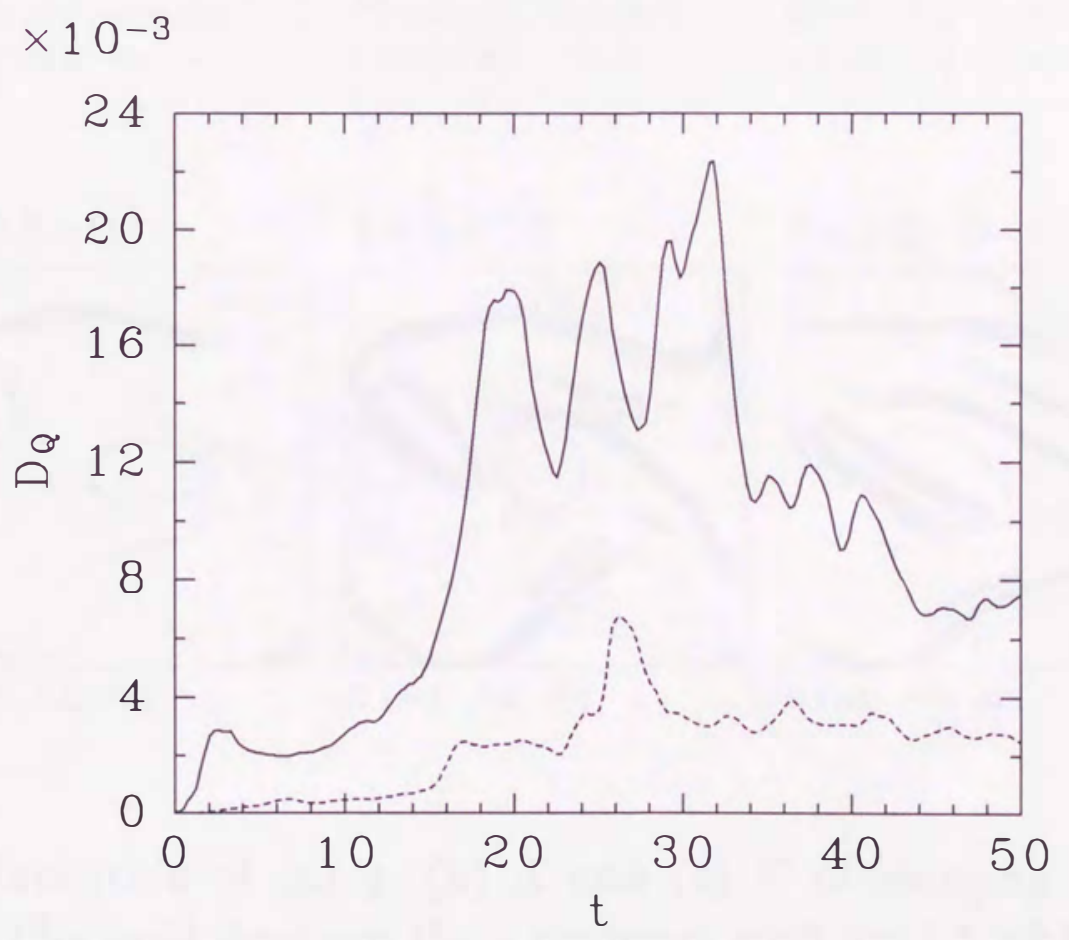

(b)

Fig. 1.7. Same as Fig. 1.6 but the evolution of enstrophy dissipation rate. 

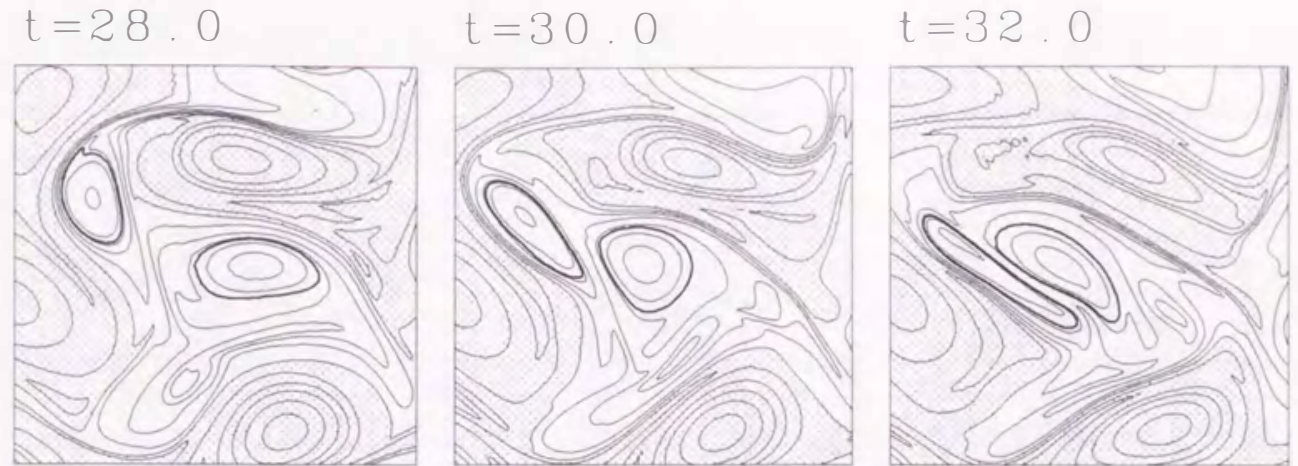

(a)
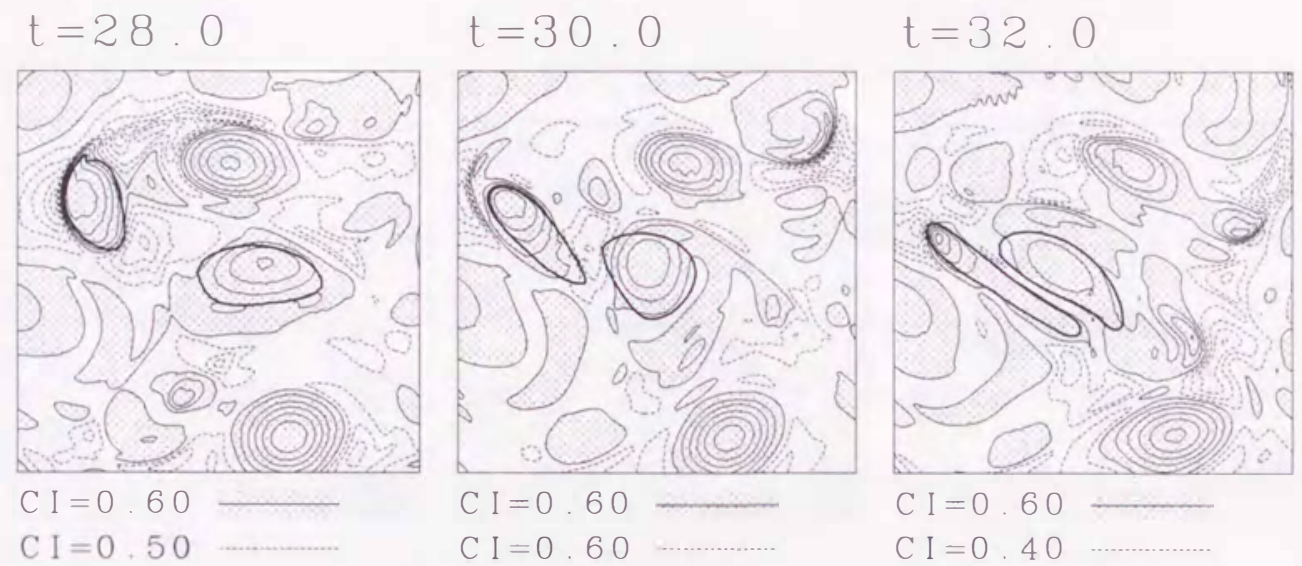

(b)
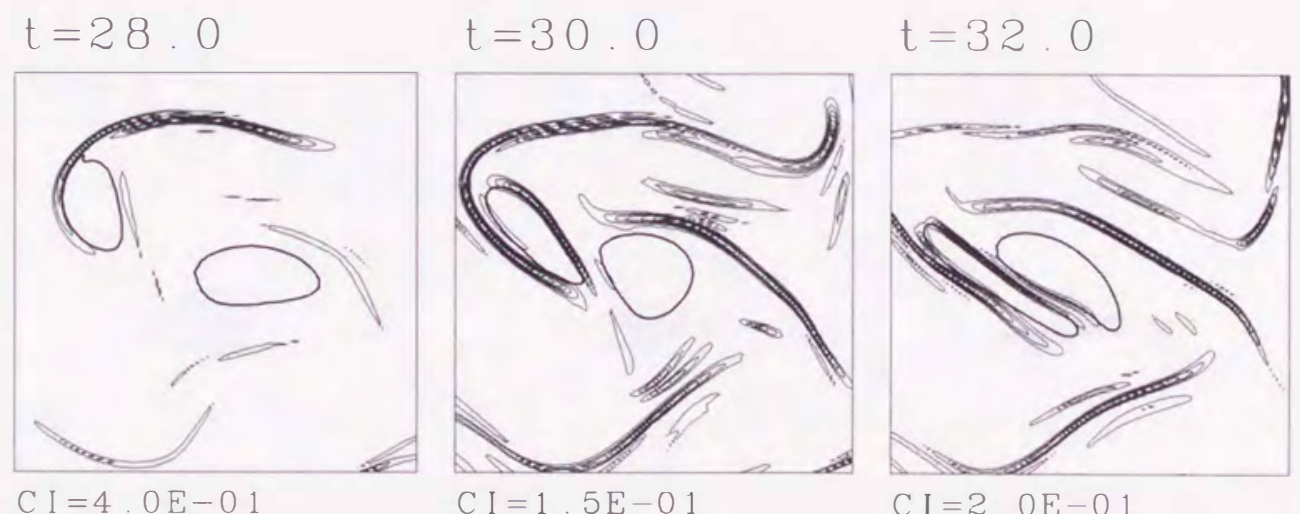

(c)

Fig. 1.8. Evolution of (a) $q$, (b) $\Lambda$ and (c) $\mathcal{P}$ of merging cyclones for Case I. The bold lines are the $q$ contours with $q=1.1$ which represent the cores of vortices. The contour intervals in (a) are the same as in Fig. 1.1(a), while those in (b) and (c) are denoted by CI. Regions of negative $\Lambda$ are shaded in (b). Only the part $-0.28 \pi<x<0.72 \pi$, $-0.89 \pi<y<0.11 \pi$ of the domain is shown. 

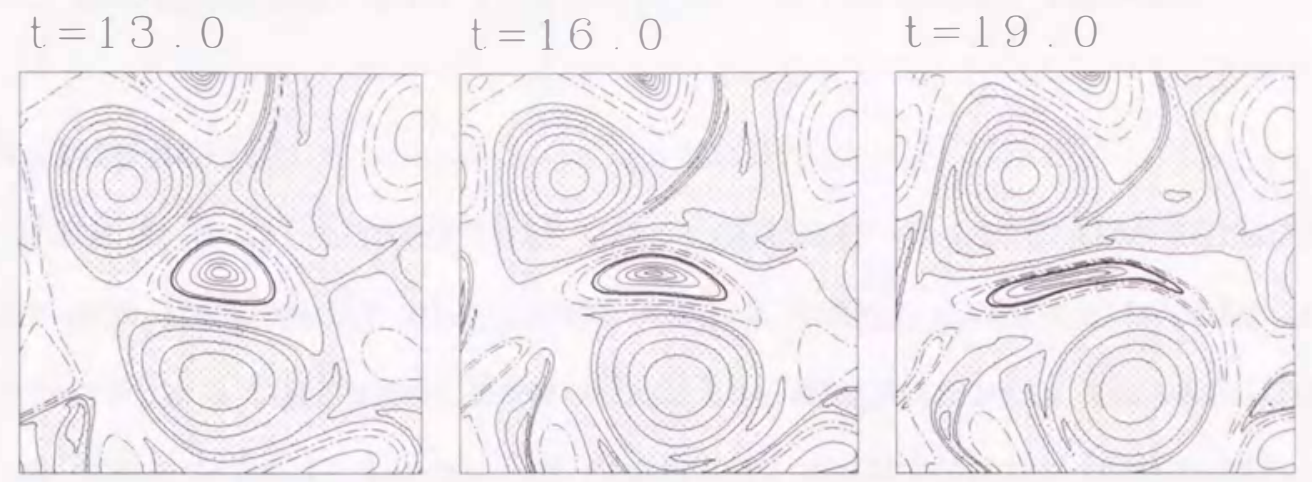

(a)

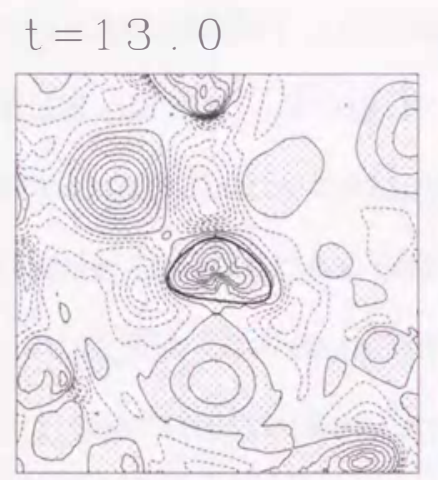

$\mathrm{C} I=0.55$

$\mathrm{C} I=0.35$
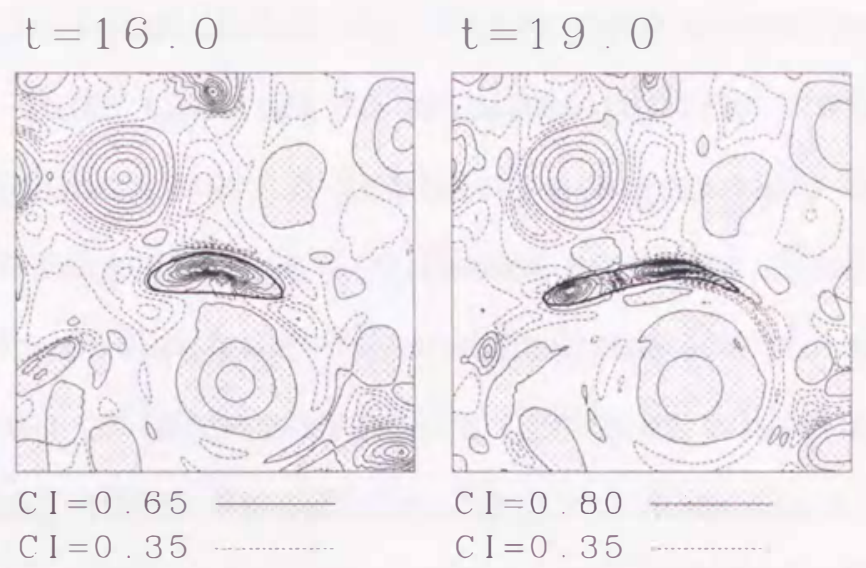

(b)

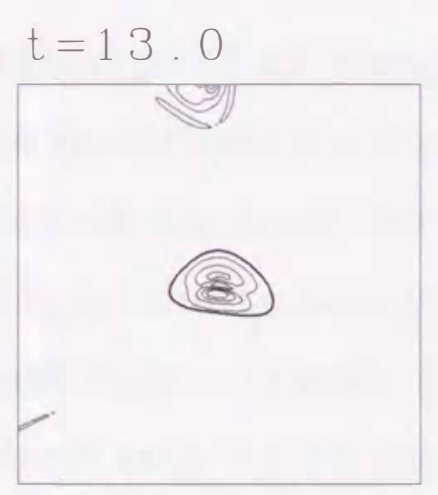

$\mathrm{C} I=1.5 \mathrm{E}+02$

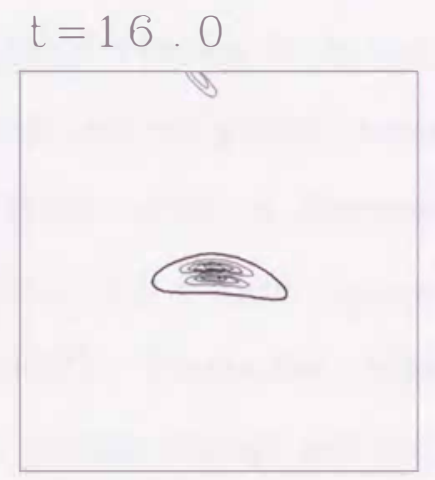

$\mathrm{C} I=5 \cdot 0 \mathrm{E}+02$

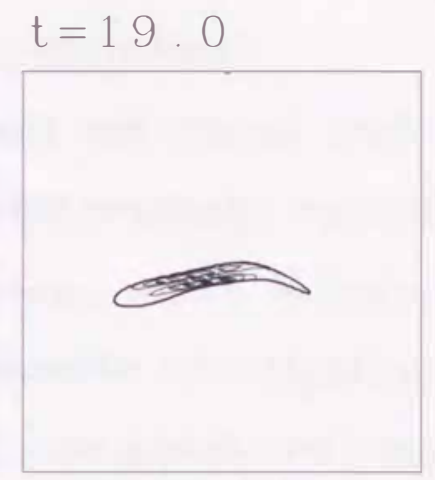

$\mathrm{C} I=1 \cdot 0 \mathrm{E}+03$

(c)

Fig. 1.9. As in Fig. 1.8 but of an elongating cyclone for Case II. The bold line is the $q$ contour with $q=3$ which represents the vortex core. The contour intervals in (a) are the same as in Fig. 1.1(b). Only the part $0<x<\pi, 0.5 \pi<y<1.5 \pi$ of the domain is shown. 
Part 2. Elongation and split-up of an isolated vortex

\subsection{Introduction}

In Part 1, it is showed that equi-contours of potential vorticity $q$ of cyclones are frequently elongated and a single cyclone breaks up into two vortices in a turbulent flow when the amplitude of vertical displacements is considerably large. In contrast, anticyclones frequently merge as in the quasi-geostrophic case. In large-amplitude cyclones, $q$ gradients are remarkably intensified just inside the vortex core where vorticity dominates strain. This is quite contrast to small-amplitude vortices or arbitrary-amplitude anticyclones, since $q$ gradients are generally intensified outside the vortex core where strain dominates vorticity. The above results suggest the existence of fundamental mechanisms for the elongation and successive break-up of large-amplitude cyclones which are not associated with the straining effect by surrounding vortices. In order to clarify these mechanisms, the relaxation process of an isolated elliptical vortex will be examined in Part 2. Here an elliptical vortex is regarded as a prototype of an elongated vortex in a turbulent flow.

The investigations about an elliptical vortex are traced back to the discovery of an exact solution with a piecewise-constant vorticity distribution in the two-dimensional Euler equations, which is known as a Kirchhoff ellipse (Lamb 1932). However, elaborate investigations have been done only in recent years using several computational methods. Dritschel (1986) examined the nonlinear evolution of a perturbed Kirchhoff ellipse by the contour dynamical method. He showed that the ellipse perturbed by the linearly neutral disturbance with the azimuthal wavenumber 2 breaks up into two vortices, while the ellipse perturbed by the linearly unstable disturbance with the wavenumber 3 or 4 generates thin filaments. Polvani et al. (1989) showed that filament generation 
occurs when perturbed vortex contours cross the separatrices emanating from saddle points in the corotating streamfunction. Melander et al. (1987) investigated the evolution of an elliptical vortex with a smooth vorticity distribution by the pseudospectral calculation. They showed that the elliptical vortex relaxes toward axisymmetry on a circulation time scale due to filament generation. They also derived a geometrical formula which gives a kinematic condition for the axisymmetrization.

In the shallow-water equations or in the frontal geostrophic equation on an $f$-plane, there exists a solution of a steadily-rotating anticyclonic elliptical vortex called the anticyclonic lens (Cushman-Roisin et al. 1985). The anticyclonic lens has a linear velocity profile and a quadratic depth profile bounded by an elliptical surface outcropping line. Cushman-Roisin (1986) investigated the linear stability of the anticyclonic lens using the frontal geostrophic equation. He showed that the anticyclonic lens is unstable when the aspect ratio (defined by the ratio of the major to minor axis) is greater than 1.8, and the egg shaped mode 3 is first destabilized. Pavia and Cushman-Roisin (1988) investigated the evolution of the anticyclonic lens numerically using the same equation. They showed that the unstable mode 3 grows initially, however, the axisymmetrization overcomes finally. The cyclonic counterpart of the anticyclonic lens has a depth profile increasing outward from its center, and its mathematical treatment is cumbersome. Thus investigations about a cyclonic elliptical vortex have been scarce. For the special case with an axisymmetric profile, Cushman-Roisin and Tang (1990) showed using the general geostrophic equation on an $f$-plane that the cyclone with considerably large vertical displacements is unstable and breaks up into a number of smaller vortices. But there seems to exist some questions about their result as described in Part 1.

In the second part of the present work, the free evolution and linear 
stability of an elliptical vortex are investigated. In order to clarify the asymmetric natures between a cyclone and an anticyclone, a symmetric vorticity profile and an asymmetric profile of surface displacement are assumed, where the surface displacement decreases outward from the vortex center. Thus the profiles are quite different from those of a lens-type vortex. Since it is difficult to obtain an analytical solution of an elliptical vortex with a smooth distribution of potential vorticity, numerical procedures are adopted.

\subsection{Initial conditions}

We consider the initial elliptical vortex with a Gaussian form for the streamfunction $\psi$ distribution. The horizontal scale is scaled by the characteristic length of the minor axis $b_{*}$, and $\psi$ is scaled by its maximum amplitude $\psi_{0}$. The velocity and time are then scaled by $\psi_{0} / b_{*}$ and $b_{*}^{2} / \psi_{0}$, respectively. In this non-dimensionalization, $\psi$ is expressed as

$$
\psi=-s \exp \left(-\frac{1}{2} \rho^{2}\right), \quad \rho^{2}=\left(\frac{x}{\lambda}\right)^{2}+y^{2} .
$$

Here $s$ denotes the polarity, where $s=1$ corresponds to a cyclone and $s=-1$ corresponds to an anticyclone, and $\lambda=a_{*} / b_{*}$, where $a_{*}$ is the characteristic length of the major axis $(1 \leq \lambda<\infty)$. The initial free surface elevation $\eta$ and the velocity potential are computed by the balance equation (1.A1b) and the simplified equation of (1.A4), respectively as explained in $\S 1.2 .4$. Here the spatial integral of $\eta$ over the model domain is given by

$$
\int \eta d x d y=\int \psi d x d y-\frac{\varepsilon}{2} \int\left(x^{2}+y^{2}\right) \Lambda_{r} d x d y,
$$

which is obtained from multiplying $x^{2}+y^{2}$ by (1.A1b), and integrating over the unbounded domain with assuming that $\eta$ and $\psi$ decay faster than $x^{-2}, y^{-2}$ in the limit $|x|,|y| \rightarrow \infty$. 
We have three parameters: the maximum surface displacement (the maximum amplitude) $\eta_{\max }$, the rotational Froude number $F$, and the aspect ratio $\lambda$. Due to gradient-wind balance, $\eta_{\max }$ is rather different between a cyclone and an anticyclone, or among vortices with different $\lambda$, although we specify the same Rossby number $\varepsilon$ and $F$. This is the reason why we adopt $\eta_{\max }$ instead of $\varepsilon$. For the axisymmetric vortex, $\eta_{\max }$ is related to $\varepsilon$ as

$$
\eta_{\max }=\varepsilon F\left(1+s \frac{1}{2} \varepsilon\right)
$$

in the present initial condition given by (2.B12b).

The half size of the domain is set at $W=10$. Interactions with vortices in the neighboring cyclic boxes are undesirable, since we are interested in the evolution of an isolated vortex. To check influences of the periodic boundary conditions, we have conducted an experiment in the domain with the double size $W=20$. We confirmed that such influences are negligible for $\lambda \leq 2.5$. The number of grid points $N$ and the hyperviscosity coefficient $\nu$ are $N=128$ and $\nu=3.6 \times 10^{-5}$ for standard simulations, and $N=256$ and $\nu=4.0 \times 10^{-6}$ for high-resolution simulations. The results of the former simulations are nearly the same with those of the latter simulations. The former simulations are used to analyze global features, and the latter simulations are used to analyze local quantities. We will mainly show the former results unless stated otherwise.

We explore the parameter space of $\eta_{\max }, F$ and $\lambda$ over $0 \leq \eta_{\max } \leq 0.9$, $0.1 \leq F \leq 4$ and $1 \leq \lambda \leq 2.5$, where the cases with $\eta_{\max }=0$ are the quasigeostrophic cases. Among those, we will mainly discuss the cases with $F=0.25$ and $\lambda=2.5$ but with different $\eta_{\max }$ for typical three cyclonic and two anticyclonic cases. The parameters for these five cases are listed in Table 2.1. In the small-amplitude case such as $\eta_{\max }=0.09$, the quasigeostrophic approximation holds well, thus the evolution is expected to 


\begin{tabular}{llcc}
\hline \hline Polarity & $\eta_{\max }$ & $\varepsilon$ & $\Omega$ \\
\hline Cyclone & 0.09 & 0.34 & 0.061 \\
Cyclone & 0.5 & 1.53 & 0.047 \\
Cyclone & 0.9 & 2.42 & 0.035 \\
Anticyclone & 0.09 & 0.39 & 0.066 \\
Anticyclone & 0.34 & 2.42 & 0.072 \\
\hline \hline
\end{tabular}

Table 2.1. Parameters for typical simulations of an isolated elliptical vortex with $F=0.25$ and $\lambda=2.5$ fixed. The last column shows the angular velocity $\Omega$ of the revolution of the elliptical vortex at the initial stage.

be similar to that in the quasi-geostrophic case. The large-amplitude anticyclone with $\eta_{\max }=0.9$ and $F=0.25$ does not satisfy gradient-wind balance.

With the above initial conditions, the shallow-water equations (1.1a), (1.1b) and the quasi-geostrophic equation (1.6) are solved numerically as explained in $\S 1.2 .3$.

\subsection{Characteristics of the evolution of an elliptical vortex}

\subsubsection{Flow evolution}

First of all, we show the evolution of equi-contours of the potential vorticity $q$ to capture global features. Here we show the typical three cyclonic and two anticyclonic cases listed in Table 2.1.

First we show the cyclonic cases. Figure 2.1(a) shows the smallamplitude case with $\eta_{\max }=0.09$. As the elliptical vortex revolves in the counter-clockwise direction, $q$ contours at the periphery are elongated and form fine spiral filaments. In the core region of the vortex, however, $q$ 
contours remain elliptical throughout the evolution. As the filamentation at the periphery develops, $q$ contours in the core region shrink (elongate) along the major (minor) axis. This tendency for approaching axisymmetry is called the axisymmetrization. The above features are quite the same with those in the quasi-geostrophic case (not shown) and similar to those in the case of Melander et al. (1987), since the quasi-geostrophic approximation holds well in this case.

Figure 2.1(b) shows the moderate-amplitude case with $\eta_{\max }=0.5$. As in the case $\eta_{\max }=0.09, q$ contours at the periphery develop into fine filaments. However, the behavior in the core region is just opposite: $q$ contours in this region elongate (shrink) along the major (minor) axis. The elongation along the major axis is not so remarkable and ceases around $t=20$. Then the contours shrink along the major axis, and the axisymmetrization prevails finally as in the previous case.

Figure 2.1(c) shows the large-amplitude case with $\eta_{\max }=0.9$. An interesting feature is observed in the early phase that the $q$ contours in the core region slightly tilt in the clockwise direction relative to those at the periphery (notice the panel at $t=4.9$ ). Simultaneously, the elongation along the major axis develops rapidly. As a consequence, the single extremum in the core region splits up into two extrema (see $t=19.7$ ), and almost all contours surrounding two extrema are cut off around $t=33$. Thus the initial single vortex completely breaks up into two corotating vortices (see $t=39.4$ ). Apart from the above dramatic behavior in the core region, the filamentation at the periphery is also observed as in the previous cases. Hereafter, the elongation along the major axis of equi- $q$ contours inside the vortex core is simply called the elongation, however, the elongation of equi- $q$ contours into fine filaments at the periphery of a vortex is called the filamentation.

Next we show the anticyclonic cases. Figure 2.1(d) shows the small- 
amplitude case with $\eta_{\max }=0.09$. Since the quasi-geostrophic approximation holds well in this case, the evolution is quite similar to that in the cyclonic case shown in Fig. 2.1(a), except that the direction of the revolution is opposite. The observed differences are insignificant: The speed of the revolution, i.e., the speed of the axisymmetrization in the anticyclonic case is slightly faster than that in the cyclonic case (compare the panels at $t=30$ ). Figure 2.1(e) shows the moderate-amplitude case with $\eta_{\max }=0.34$. The evolution is similar to that in the anticyclonic case with $\eta_{\max }=0.09$, but is quite different from that in the cyclonic case with moderate $\eta_{\max }$ shown in Fig. 2.1(b). This is a manifestation of the asymmetric nature originated from the nonlinear divergence of the continuity equation $(1.1 \mathrm{~b})$. In the moderate-amplitude cyclonic case, the initial elongation greatly suppresses the axisymmetrization. In the anticyclonic case, however, the axisymmetrization persists throughout the evolution without showing any sign of the elongation.

\subsubsection{Diagnostic ellipse}

For the following quantitative analyses, we introduce the $q$-ellipse and $\Psi$-ellipse according to Melander et al. (1987). The $q$-ellipse and $\Psi$-ellipse are the ellipses approximate to a contour of the potential vorticity $q$ and that of the transport streamfunction $\Psi$, respectively in the core region. The core is the region where vorticity exceeds strain, thus $q$ contours will remain elliptical. The aspect ratio $\lambda_{q}\left(\lambda_{\Psi}\right)$ and the orientation $\theta_{q}\left(\theta_{\Psi}\right)$ are evaluated for the $q$-ellipse ( $\Psi$-ellipse). Here the aspect ratio is defined by the ratio of the major to minor axis. The detailed definitions and numerical procedures are described in the Appendix 2.A.

Figure 2.2 shows the evolution of $\lambda_{q}$ for the typical three cyclonic cases. The evolution for the anticyclonic cases (not shown) is qualitatively the same as that for the cyclonic case $\eta_{\max }=0.09$. In the case 
$\eta_{\max }=0.09, \lambda_{q}$ decreases monotonically by $t \sim 40$, which is a manifestation of the axisymmetrization. In the case $\eta_{\max }=0.5, \lambda_{q}$ increases in the initial phase by $t \sim 21$, which is a manifestation of the elongation. Then $\lambda_{q}$ begins to decrease. After $t \sim 30, \lambda_{q}$ decreases rapidly and it continues by $t \sim 56$, which means that the axisymmetrization prevails finally. In the case $\eta_{\max }=0.9, \lambda_{q}$ increases monotonically, and the vortex breaks up completely as indicated by the discontinuity of the curve around $t=33$. The slight decrease of $\lambda_{q}$ during $26 \lesssim t \lesssim 33$ has no physical meanings, since $q$ contours are considerably distorted from elliptical shapes.

\subsubsection{Dependences on the parameters}

We define the elongation rate $\sigma_{e}$, and examine its dependences on the parameters, the maximum surface displacement $\eta_{\max }$, the aspect ratio $\lambda$ and the rotational Froude number $F$. We assume that the major axis $a$ and the minor axis $b$ of the $q$-ellipse change as $a \propto e^{\sigma_{e} t}$ and $b \propto e^{-\sigma_{\epsilon} t}$, respectively. Then the aspect ratio $\lambda_{q}$ increases (if $\sigma_{e}>0$ ) or decreases (if $\left.\sigma_{e}<0\right)$ as $\lambda_{q} \propto e^{2 \sigma_{e} t}$. Negative $\sigma_{e}$ means the axisymmetrization. Here $\sigma_{e}$ is obtained by the least-squares fit to the initial stage of the $t-\lambda_{q}$ curve.

As represented by the typical three cases shown in Figs. 2.1 and 2.2, the evolution of cyclonic elliptical vortices is classified into three regimes (A, AE and SE) depending on $\eta_{\max }$ with $F$ and $\lambda$ fixed. Figure 2.3 shows $\sigma_{e}$ as a function of $\eta_{\max }$ for the cases with $\lambda=2.5$ and $F=0.25$. Over the explored parameter range, $\sigma_{e}$ increases almost linearly from negative to positive values with increasing $\eta_{\max }$. The elongation occurs initially when $\eta_{\max }$ exceeds the first critical value $\eta_{c 1}=0.23$, while the axisymmetrization persists throughout the evolution (the regime A) when $\eta_{\max }$ is below this value. In addition, the elongation causes the split-up of a vortex core into two (the regime SE) when $\eta_{\max }$ exceeds the second critical value $\eta_{c 2}\left(0.5<\eta_{c 2}<0.6\right)$. On the other hand, the elongation ceases at some 
stage and the axisymmetrization excels finally (the regime $\mathrm{AE}$ ) when $\eta_{c 1}<\eta_{\max }<\eta_{c 2}$. In contrast, the axisymmetrization always occurs for anticyclonic elliptical vortices irrespective of the values of $\eta_{\max }, F$ and $\lambda$ (the regime $\mathrm{A}$ ).

Next we show $\sigma_{e}$ as a function of $\lambda$ for the cyclonic vortices with $\eta_{\max }=0.9$ and $F=0.25$ in Fig. 2.4. The elongation always occurs over the explored range of $\lambda$ except for the axisymmetric case $(\lambda=1)$. The elongation rate $\sigma_{e}$ increases gradually with increasing $\lambda$. The regime $\mathrm{AE}$ occurs when $\lambda$ is smaller than a critical value around 1.5 , while the regime $\mathrm{SE}$ occurs when $\lambda$ exceeds this value. The elongation rate approaches zero as $\lambda \rightarrow 1$, and the axisymmetric vortex is stable. This is quite contrast with the result of Cushman-Roisin and Tang (1990). In their case, the large-amplitude axisymmetric cyclone with $\eta_{\max }=1$ and $F=9$ (in our non-dimensional form) breaks up into many smaller vortices. Since they did not describe their initial condition, we cannot re-examine their result. Anyway in the present case, axisymmetric cyclones are stable at least for $\eta_{\max }=0.9$ and $0.1 \leq F \leq 9$. The linear stability of axisymmetric vortices is discussed analytically in the Appendix 2.B.

Figure 2.5 shows $\sigma_{e}$ as a function of $F$ for the cyclonic elliptical vortices with $\eta_{\max }=0.9$ and $\lambda=2.5$. The elongation rate $\sigma_{e}$ decreases monotonically with increasing $F$. The regime SE occurs when $F$ is below the critical value $F_{c}\left(1<F_{c}<2\right)$, while the regime $\mathrm{AE}$ occurs when $F$ exceeds $F_{c}$. This demonstrates that the elongation is more and more suppressed as the horizontal scale of a vortex is increased. The flow regimes for the cyclonic elliptical vortices with $\lambda=2.5$ are summarized on the diagram of $\eta_{\max }$ versus $F$ in Fig. 2.6. The first critical value $\eta_{c 1}$ and the second critical value $\eta_{c 2}$ at given $F$ increase with increasing $F$, and the regime SE never occurs when $F>F_{c}$. 


\subsection{Kinematic description of the elongation and split-up}

\subsubsection{Axisymmetrization/Elongation principle}

Melander et al. (1987) proposed an interesting formula which gives a kinematic condition not only for the axisymmetrization but also for the elongation. The key concept is the geometrical relation between the $q$ ellipse and $\Psi$-ellipse. To quantify this, we introduce the difference angle $\theta_{l}$ as

$$
\theta_{d}=\theta_{q}-\theta_{\Psi},
$$

where $\theta_{q}$ and $\theta_{\Psi}$ are the orientations of the $q$-ellipse and $\Psi$-ellipse, respectively. The axisymmetrization principle is stated as follows: If $\theta_{d}>0$, that is, if the $\Psi$-ellipse lags behind the $q$-ellipse, the aspect ratio $\lambda_{q}$ decreases. On the other hand, if $\theta_{d}<0$, that is, if the $q$-ellipse lags behind the $\Psi$-ellipse, $\lambda_{q}$ increases. Thus this principle is formulated as

$$
\frac{d \lambda_{q}}{d t} \cdot \theta_{d}<0
$$

The physical explanation of this principle is as follows. We note that the tangent to an equi- $\Psi$ contour approximately gives the direction of a velocity vector as implied by (1.3). We assume that the $\Psi$-ellipse lags behind the $q$-ellipse (i.e., $\theta_{d}>0$ ) as shown in Fig. 2.7(a). The velocity vector at $\mathrm{A}$ has an inward projection on the major axis of the $q$-ellipse, which advects potential vorticity in the direction to the origin $O$. On the other hand, the velocity vector at $\mathrm{B}$ has an outward projection on the minor axis of the $q$-ellipse, which advects potential vorticity in the direction opposite to the origin. Hence the aspect ratio $\lambda_{q}$ of the $q$-ellipse will decrease (i.e., $\frac{d \lambda_{q}}{d t}<0$ ). In the opposite geometry that the $q$-ellipse lags behind the $\Psi$-ellipse (i.e., $\theta_{d}<0$ ) as shown in Fig. 2.7(b), a similar argument explains the increase of $\lambda_{q}$ (i.e., $\frac{d \lambda_{q}}{d t}>0$ ).

We apply the axisymmetrization principle to the present shallowwater cases. In Fig. 2.2 the evolution of $\theta_{d}$ is displayed as well. In the 
case $\eta_{\max }=0.09, \theta_{d}$ is positive by $t \sim 40$, and in accordance with this, $\lambda_{q}$ decreases rapidly. The axisymmetrization principle holds well in such small-amplitude cases. In the case $\eta_{\max }=0.5, \theta_{d}$ is negative by $t \sim 30$ and $\lambda_{q}$ actually increases by $t \sim 20$, although the time lag is observed: $\lambda_{q}$ slightly decreases when $\theta_{d}$ remains negative. In the case $\eta_{\max }=0.9$, except at the later stage $26 \lesssim t \lesssim 33$, the relation $(2.4)$ holds quite well: $\theta_{d}$ is negative and $\lambda_{q}$ increases rapidly by $t \sim 25$. The above two cases show that the axisymmetrization principle can be applicable to the elongation as the elongation principle.

The axisymmetrization/elongation principle cannot reveal how the geometry for the axisymmetrization or elongation is realized. The mechanism for the axisymmetrization is described in the Appendix 2.C. The mechanisms for the elongation and successive split-up will be discussed in the following subsections.

\subsubsection{Stagnation point concept}

The mechanism for deformation of $q$ contours, such as the elongation (occurring in the regime $\mathrm{SE}$ or $\mathrm{AE}$ ) and filamentation (occurring in all regimes), can be well described by considering the nature of stagnation (critical) points which $q$ contours surround (Melander et al. 1987). Stagnation points are the points where the velocity is zero in an appropriate frame. Here we adopt a frame rotating with an elliptical vortex (hereafter abbreviated as the corotating frame). The velocity $\boldsymbol{u}_{c}=\left(u_{c}, v_{c}\right)$ in the corotating frame is related to the velocity $\boldsymbol{u}=(u, v)$ in the frame fixed to the $f$-plane as

$$
\left(u_{c}, v_{c}\right)=(u+\Omega y, v-\Omega x)
$$

where $\Omega$ is an appropriate angular velocity.

We do not have a unique way of defining $\Omega$, since the present initial 
conditions are not steady solutions. That is, $q$ contours with different $q$ rotate with different angular velocity. Here we adopt the same procedure as in Melander et al. (1987) to obtain $\Omega$ a posteriori: $\Omega$ is numerically determined by the time-differentiation of the orientation $\theta_{\Psi}$ of the $\Psi$ ellipse. The detail procedure is described in the Appendix 2.A. The obtained values of $\Omega$ for the typical cases are listed in Table 2.1.

First we briefly review the theory of stagnation points in two-dimensional compressible flows (Perry and Chong 1987). We expand the velocity in a Taylor series around a stagnation point and adopt only the lowest-order terms. The equation of motion of a fluid particle is approximately

$$
\frac{d \boldsymbol{X}}{d t}=\nabla \boldsymbol{u}_{c} \cdot \boldsymbol{X}
$$

where $\boldsymbol{X}=(X, Y)$ is the position of the fluid particle relative to the stagnation point, and $\nabla \boldsymbol{u}_{c}$ is the velocity-gradient tensor defined at the stagnation point as

$$
\nabla \boldsymbol{u}_{c}=\left(\begin{array}{ll}
\frac{\partial u_{c}}{\partial x} & \frac{\partial u_{c}}{\partial y} \\
\frac{\partial v_{c}}{\partial x} & \frac{\partial v_{c}}{\partial y}
\end{array}\right)=\left(\begin{array}{cc}
s_{11} & s_{12}-\frac{1}{2} \zeta_{c} \\
s_{12}+\frac{1}{2} \zeta_{c} & s_{22}
\end{array}\right) .
$$

Here

$$
s_{12}=\frac{1}{2}\left(\frac{\partial v_{c}}{\partial x}+\frac{\partial u_{c}}{\partial y}\right), \quad \zeta_{c}=\frac{\partial v_{c}}{\partial x}-\frac{\partial u_{c}}{\partial y} .
$$

The above equation has solutions of the form $\boldsymbol{X}=\boldsymbol{X}_{0} e^{\sigma_{ \pm} t}$, where $\boldsymbol{X}_{0}=$ $\left(X_{0}, Y_{0}\right)$ is the initial position, and $\sigma_{ \pm}$are the eigenvalues of $\nabla \boldsymbol{u}_{c}$ given by

$$
\sigma_{ \pm}=s_{d} \pm \sqrt{\Lambda_{c}}
$$

Here

$$
s_{d}=\frac{1}{2}\left(s_{11}+s_{22}\right),
$$




$$
\Lambda_{c}=\frac{1}{4}\left(s_{11}-s_{22}\right)^{2}+s_{12}^{2}-\frac{1}{4} \zeta_{c}^{2} .
$$

For an unsteady flow the velocity is time-dependent. Thus the solution trajectories obtained from (2.6) give instantaneous streamlines. However, if we assume the velocity to be frozen for all times, the instantaneous solution trajectories coincide with the trajectories of fluid particles. In this case, since potential vorticity is conserved with fluid particles in an inviscid case, the motion of particles can be directly related to the motion of $q$ contours surrounding a stagnation point. Off course this is not exactly true for all times, since potential vorticity is not a passive scalar but a dynamical quantity as implied by the relation (2.C1) in the quasi-geostrophic limit. However, as far as we consider a short-time evolution of a $q$ contour under given velocity field, we can neglect the change of velocity due to the redistribution of potential vorticity. This may be validated since potential vorticity is a local quantity compared with velocity as discussed in $§ 1.5$.

Types of stagnation points are classified depending on the signs of $\Lambda_{c}$ and $s_{d}$, and on the relative magnitude of $\Lambda_{c}$ and $s_{d}^{2}$ as summarized in Fig. 2.8. If $\Lambda_{c}>0$ and $\left|s_{d}\right|<\sqrt{\Lambda_{c}}$, that is, if $\sigma_{+}(>0)$ and $\sigma_{-}(<0)$ are real, the stagnation point is a saddle. A $q$ contour which encloses a saddle elongates in some direction and shrinks in the direction perpendicular to the elongating direction. In the limit $t \rightarrow \infty$, the elongating direction coincides with the eigenvector associated with $\sigma_{+}$. If $\Lambda_{c}>0$ and $\left|s_{d}\right|>\sqrt{\Lambda_{c}}$, the stagnation point is an unstable node (when $\sigma_{+}>\sigma_{-}>0$ ) or a stable node (when $\sigma_{-}<\sigma_{+}<0$ ). But these cases may be discarded, because the initial conditions are in gradient-wind balance. If $\Lambda_{c}<0, \sigma_{ \pm}$ are imaginary, and the stagnation point is a stable focus (when $s_{d}<0$ ), an unstable focus (when $s_{d}>0$ ), or a center (when $s_{d}=0$ ). Since the 
divergence is weak (ie., $s_{d} \ll 1$ ) except in the core region, the stagnaion points with $\Lambda_{c}<0$ outside the core region are approximately centers. The motion of a $q$ contour surrounding a center will be discussed in $§ 2.4 .4$.

2.4.3. Kinematic condition for the split-up

For the present elliptical vortex, the geometry of stagnation points at $t=0$ is shown in Fig. 2.9. There exist two saddles $S_{1}$ and $S_{2}$ on the major axis, two centers $C_{g 1}$ and $C_{g 2}$ on the minor axis, and another center $O$ at the origin. The four solid lines $o_{1}, o_{2}, i_{1}$ and $i_{2}$ are the streamlines (called the separatrices) which connect the saddles $S_{1}$ and $S_{2}$. This initial geometry is quite the same with that in the quasi-geostrophic cases (Melander et al. 1987; Polvani et al. 1989).

Next we examine the evolution of cyclonic vertices in the corotating frame. For simplicity we first display the streamfunction (corotating streamfunction) instead of the streamlines. The flow field can be described approximately by the streamfunction throughout the domain for the case with small vertical displacements and only outside the core region for the case with large displacements, since the divergence is negligibly small. However, for the case with large displacements, the divergence is no longer small inside the core region. Thus we next compare the avolotion of the streamlines with that of the streamfunction in this case. We note that the streamlines represent the total velocity field, while the streamfunction represents the rotational part of the velocity field. The corotating streamfunction $\psi_{c}$ is defined as

$$
\psi_{c}=\psi-\frac{\Omega}{2}\left(x^{2}+y^{2}\right) .
$$

In the non-divergent case, $\Lambda_{c}$ is reduced to $\Lambda_{r c}$ given by

$$
\Lambda_{r c}=\left(\frac{\partial^{2} \psi_{c}}{\partial x \partial y}\right)^{2}-\frac{\partial^{2} \psi_{c}}{\partial x^{2}} \frac{\partial^{2} \psi_{c}}{\partial y^{2}} .
$$

53 
The negative of $\Lambda_{r c}$ has a geometrical meaning, that is, $-\Lambda_{r c}$ is identical with the Gaussian curvature of $\psi_{c}$ at the point with $\nabla \psi_{c}=0$. If $-\Lambda_{r c}>0$ at the center $O$ of a vortex, the $\psi_{c}$ field has a single extremum inside the core region, which means that the vortex is single. On the other hand, if $-\Lambda_{r c}<0$ at $O$, the $\psi_{c}$ field has at least two extrema inside the core region, which means that the vortex locally splits up. Hence we can give an alternative definition for the local split-up of the core by the change of the sign of $\Lambda_{r c}$ at $O$.

Figure 2.10 shows the evolution of the $\psi_{c}$ field and the two $q$ contours representing the core and periphery for the cyclonic elliptical vortices with $\eta_{\max }=0.09$ and $\eta_{\max }=0.9$. These two cases exhibit the same characters at $t=0$. The $q$ contour in the core region is confined to the inner separatrices $i_{1}$ and $i_{2}$. Therefore this contour will remain elliptical as long as the stagnation point $O$ does not change its nature. On the other hand, the $q$ contour at the periphery encloses the saddles $S_{1}$ and $S_{2}$, therefore will be elongated along the outer separatrices $o_{1}$ and $o_{2}$. In spite of the above similarities at $t=0$, the evolution for $\eta_{\max }=0.9$ (the regime SE) is quite different from that for $\eta_{\max }=0.09$ (the regime A). In the case $\eta_{\max }=0.09$, as expected, the $q$ contour at the periphery is actually elongated along the outer separatrices, and wraps around the centers $C_{g 1}$ and $C_{g 2}$. The $q$ contour in the core region always stays within the inner separatrices, and remains elliptical throughout the evolution. In the case $\eta_{\max }=0.9$, the evolution at the periphery is similar to that in the case $\eta_{\max }=0.09$. However, a dramatic phenomenon takes place inside the inner separatrices. That is, the sign of the Gaussian curvature at $O$ changes from a positive to negative value around $t=12$, which means the local split-up of the vortex core. Actually in accordance with this, there appear two extrema also in the $q$ field around $t=13$ (not shown).

In order to clarify the mechanism for the split-up of the vortex core 
in the case $\eta_{\max }=0.9$, we examine the evolution of the streamlines in Fig. 2.11. A surprising phenomenon is observed in the total velocity field at a stage much earlier than the time $t \sim 12$ of the local split-up. Namely, as the core elongates, the stagnation point $O$ first becomes a stable focus, and then changes into a saddle and two foci appear around $t=2$ (not shown, but notice the panel at $t=3.9$ ). We note that the Gaussian curvature of $\psi_{c}$ remains positive at this time. After this change the stagnation point again becomes a stable focus for a while $5 \lesssim t \lesssim 10$, but the elongation still develops. This is because the flow pattern still satisfies the geometry for the elongation. The time lag between the time of the change into a saddle in the total velocity field and that of the local splitup of the core suggests an essential role of an ageostrophic (irrotational) motion. Under the geometry for the elongation, a fluid particle immersed in the second or fourth quadrant of the ellipse is advected outward. Since the fluid depth increases outward, this particle gets larger vorticity due to the conservation of potential vorticity. This change of vorticity is comparable to the planetary vorticity when $\eta_{\max }$ is large. Thus a disturbance associated with such a motion has an ageostrophic nature (Griffiths et al. 1982). The nature of such a disturbance will be discussed in $\S 2.5$.

The above phenomenon exhibits quite a contrast to the break-up of a vortex in the purely geostrophic case (Flierl 1988), where the time $t_{\text {ch }}$ of the change into a saddle coincides with the time $t_{\mathrm{sp}}$ of the local split-up of the vortex core. In the present case, $t_{\mathrm{ch}}$ and $t_{\mathrm{sp}}$ delay with decreasing $\eta_{\max }$. An interesting feature is that the time lag $t_{\mathrm{sp}}-t_{\mathrm{ch}}$ also shortens with decreasing $\eta_{\max }$. Figure 2.12 shows $t_{\mathrm{ch}} / t_{\mathrm{sp}}$ as a function of $\eta_{\max }$. The scaled time $t_{\mathrm{ch}} / t_{\mathrm{sp}}$ approaches unity as $\eta_{\max }$ approaches the second critical value $\eta_{c 2}$ between the regime $\mathrm{SE}$ and $\mathrm{AE}$ from above. And the stagnation point no longer changes into a saddle throughout the evolution when $\eta_{\max }<\eta_{c 2}$. In all cases of the regime SE shown in Figs. 2.4 
and 2.6, the change into a saddle always occurs before the local split-up. This fact demonstrates that the appearance of a saddle inside the core is at least a necessary condition for the split-up.

\subsubsection{Kinematic condition for the elongation}

By considering the short-time deformation of a $q$ contour around the stagnation point $O$, we can derive a kinematic condition for the initial axisymmetrization (in the regime $\mathrm{A}$ ) or the initial elongation (in the regime $\mathrm{AE}$ or $\mathrm{SE})$.

In the neighborhood of the origin $O$, the present elliptical flow is approximately a linear shear flow expressed as

$$
u_{c} \sim-\frac{\partial \psi_{c}}{\partial y} \sim(-s+\Omega) y, v_{c} \sim \frac{\partial \psi_{c}}{\partial x} \sim\left(s \lambda^{-2}-\Omega\right) x .
$$

Namely, $s_{11}$ and $s_{22}$ are nearly zero, and $s_{12}$ and $\zeta_{c}$ are dominant at $O$. Thus we assume $s_{11}=s_{22}=0$ at $O$, and examine the motion of a $q$-ellipse in a cyclonic flow with constant strain $s_{12}(<0)$ and vorticity $\zeta_{c}(>0)$. In addition, we assume $\left|s_{12}\right|<\zeta_{c} / 2$, that is, the stagnation point $O$ is a center. We do not lose generality by the last assumption, since the time scale $s_{d}^{-1}$ of divergence is much longer than the time rate of change of strain and vorticity when the stagnation point is a focus. Under this assumption, Eq. (2.6) has the solution of the form,

$$
\left(\begin{array}{l}
x \\
y
\end{array}\right)=\left(\begin{array}{cc}
\cos \sigma t & -\lambda_{s} \sin \sigma t \\
\lambda_{s}^{-1} \sin \sigma t & \cos \sigma t
\end{array}\right)\left(\begin{array}{l}
x_{0} \\
y_{0}
\end{array}\right),
$$

where $\sigma$ is the imaginary part of the eigenvalue of $\nabla \boldsymbol{u}_{c}$ given by

$$
\sigma=\sqrt{\frac{1}{4} \zeta_{c}^{2}-s_{12}^{2}}
$$


$\lambda_{s}$ is a characteristic aspect ratio defined by

$$
\lambda_{s}=\sqrt{\frac{\frac{1}{2} \zeta_{c}-s_{12}}{\frac{1}{2} \zeta_{c}+s_{12}}},
$$

and $(x, y)=\left(x_{0}, y_{0}\right)$ is the initial position. Thus the $q$-ellipse with the aspect ratio $\lambda_{q}=a / b$ at $t=0$,

$$
\frac{x_{0}^{2}}{a^{2}}+\frac{y_{0}^{2}}{b^{2}}=1,
$$

is distorted to the ellipse expressed by

$$
A x^{2}+2 B x y+C y^{2}=1,
$$

if we assume that potential vorticity is a passive scalar. Here

$$
\begin{aligned}
& A=\frac{1}{a^{2}} \cos ^{2} \sigma t+\frac{1}{\lambda_{s}^{2} b^{2}} \sin ^{2} \sigma t, \\
& B=\frac{\lambda_{s}}{a^{2}} \sin \sigma t \cos \sigma t\left(1-\frac{\lambda_{q}^{2}}{\lambda_{s}^{2}}\right), \\
& C=\frac{\lambda_{s}^{2}}{a^{2}} \sin ^{2} \sigma t+\frac{1}{b^{2}} \cos ^{2} \sigma t .
\end{aligned}
$$

The above $q$-ellipse behaves in an oscillatory manner around the frozen $\Psi$ distribution. The principal axes of the $q$-ellipse tilt in the clockwise direction relative to those of the $\Psi$-ellipse with elongating (shrinking) along the major (minor) axis during a half of the period $\pi / \sigma$, and tilts in the counter-clockwise direction with shrinking (elongating) along the major (minor) axis during another half of the period. The above behavior is quite consistent with the axisymmetrization/elongation principle discussed in $\S 2.4 .1$. We note that the sign of $B$ determines whether the ellipse tilts in the clockwise direction or in the counter-clockwise direction in the initial phase, $0<t<\pi / 2 \sigma$. If $\lambda_{q}>\lambda_{s}$, the $q$-ellipse tilts initially in 
the counter-clockwise direction, while if $\lambda_{q}<\lambda_{s}$, the $q$-ellipse tilts initially in the clockwise direction.

Figure 2.13 shows $\lambda_{q}$ and $\lambda_{s}$ at $t=0$ as a function of $\eta_{\max }$ for the cyclonic elliptical vortices. The aspect ratio $\lambda_{q}$ decreases with increasing $\eta_{\max }$, since the contribution from the denominator $h$ increases relative to the numerator $1+\varepsilon \zeta$ in the definition of $q$. On the other hand, $\lambda_{s}$ increases with increasing $\eta_{\max }$, since the irrotational component of the strain $s_{12}$ increases. Thus there exists a threshold 0.41 for $\eta_{\max }$. When $\eta_{\max }<0.41$, $\lambda_{q}$ exceeds $\lambda_{s}$, that is, the initial configuration favors the axisymmetrization. On the other hand, when $\eta_{\max }>0.41, \lambda_{s}$ exceeds $\lambda_{q}$, that is, the initial configuration favors the elongation. This threshold 0.41 is somewhat disagree with the first critical value $\eta_{c 1}=0.23$ between the regime $\mathrm{A}$ and $\mathrm{AE}$. However, the above kinematic condition qualitatively explains the difference between the regime $\mathrm{A}$ and the regimes $\mathrm{AE}, \mathrm{SE}$.

\subsection{Linear dynamics of the elongation}

The kinematic approach described in the previous section well provides the conditions for the elongation and successive split-up. However, this approach cannot reveal the mechanisms for the growth of the elongation and the successive change of the type of the stagnation point $O$, since potential vorticity is assumed to be a passive scalar. As a first step to include the dynamical aspect, that is, the effect of the change of the velocity field due to the redistribution of potential vorticity, we examine linear dynamics of an elliptical vortex. In a linear theory, the advection of the basic potential vorticity by the disturbance velocity (the third term in $(2.19))$ is essential for shear instability, while in the kinematic approach, only the advection of potential vorticity by the basic velocity (the second term in (2.19)) is considered. Before describing the method and results, we note a special limitation for the present analysis. That is, the basic 
elliptical vortex we will adopt here is not a steady solution. Thus the basic vortex cannot be defined in the corotating frame but in the frame fixed to the $f$-plane. However, the elongation we are interested in here mainly takes place inside the core region. In the vicinity of the origin $O$, the correction $(\Omega y,-\Omega x)$ (due to the rigid rotation of the frame) to the basic velocity $(u, v)$ in $(2.5)$ is small. Therefore this shortcoming will not be so serious.

\subsubsection{Numerical method for the linear stability analysis}

The inviscid, linearized shallow-water equations are

$$
\begin{aligned}
& \varepsilon\left[\partial_{t} \boldsymbol{u}+(\boldsymbol{U} \cdot \nabla) \boldsymbol{u}+(\boldsymbol{u} \cdot \nabla) \boldsymbol{U}\right]+\hat{z} \times \boldsymbol{u}+\nabla \eta=0, \\
& \partial_{t} h+\nabla \cdot(\boldsymbol{U} h+H \boldsymbol{u})=0,
\end{aligned}
$$

where $\boldsymbol{U}=(U, V)$ and $H$ are the velocity and fluid depth of the basic field, respectively, and $\boldsymbol{u}=(u, v), \eta$ and $h=\varepsilon F \eta$ are the velocity, free surface elevation and fluid depth of the disturbance field, respectively. The potential vorticity $q$ of the disturbance field is defined as

$$
q=\frac{\varepsilon \zeta-Q h}{H},
$$

where $Q$ is the potential vorticity of the basic field and $\zeta=\partial_{x} v-\partial_{y} u$ is the vorticity of the disturbance field. The disturbance $q$ yields

$$
\partial_{t} q+(\boldsymbol{U} \cdot \nabla) q+(\boldsymbol{u} \cdot \nabla) Q=0 .
$$

Here the basic fields are the initial conditions employed in the numerical simulation. In this case, Eqs. (2.18a) and (2.18b) are non-separable. Thus we solve these equations directly using a following numerical procedure. We assume the same square domain with doubly periodic boundary conditions as in the simulation. The equations (2.18a) and (2.18b) are 
projected on a basis of the double Fourier series truncated at a finite wavenumber $\frac{\pi}{W} M$ ( $M$ is an integer) in each direction. If we assume that time $t$ is separable as $(\boldsymbol{u}, \eta) \propto e^{\sigma t},(2.18 \mathrm{a})$ and $(2.18 \mathrm{~b})$ are reduced to the eigenvalue problem about the eigenvalue $\sigma$. The basic fields have the inversion symmetry about the origin as

$$
H \rightarrow H,(U, V) \rightarrow(-U,-V) \quad \text { as } \quad(x, y) \rightarrow(-x,-y),
$$

and the processes of the elongation and axisymmetrization observed in the simulation keep this symmetry. Thus we impose the same symmetry on the disturbance fields. The eigenvalues and eigenvectors are solved numerically by the double QR method and inverse method, respectively. The number $M$ is 21 , which corresponds to the grid points 64 in each direction for the dealiased pseudospectral method. This resolution is poorer than that in the simulation. However, the computation with $M=42$ is impossible due to the limitation of the storage of the supercomputer used in the present study. In order to increase the resolution of a basic vortex, the half size of the domain is set at $W=6$. In this narrow domain, influences of the boundary conditions are negligible for $\lambda \leq 2$.

\subsubsection{Growth rate}

Over the wide parameter range explored, there exist the most unstable disturbances with a similar modal structure. First of all, we show the dependences of the growth rate $\sigma_{r}=\operatorname{Re} \sigma$ of this disturbance on the parameters $\eta_{\max }, \lambda$ and $F$.

Figure 2.14 shows $\sigma_{r}$ as a function of $\eta_{\max }$ for the basic cyclonic and anticyclonic vortices with $\lambda=1.5$ and $F=0.25$. Note that anticyclonic basic states do not exist when $\eta_{\max }$ is large (about $\eta_{\max }>0.22$ ), since we assume gradient-wind balance. There exist notable differences between cyclones and anticyclones. The growth rate $\sigma_{r}$ for cyclones is much larger 
than that for anticyclones when $\eta_{\max }$ is not so small. The dependences on $\eta_{\max }$ are also quite different. For cyclones, $\sigma_{r}$ increases almost linearly with increasing $\eta_{\max }$, while for anticyclones, $\sigma_{r}$ decreases slightly with increasing $\eta_{\max }$ up to the solvability boundary. When $\eta_{\max }$ is very small, the quasi-geostrophic approximation holds well. Thus $\sigma_{r}$ for cyclones is nearly the same as $\sigma_{r}$ for anticyclones. The above result is consistent qualitatively with the cyclonic result of the numerical simulation, where the elongation rate increases almost linearly with increasing $\eta_{\max }$. The result for $\lambda=1.5$ (not shown) is qualitatively similar to that for $\lambda=2.5$ shown in Fig. 2.3, except that the first critical value is 0.38 and the regime SE never occurs.

Figure 2.15 shows $\sigma_{r}$ as a function of $\lambda$ for the basic cyclonic vortices with $\eta_{\max }=0.9$ and $F=0.25$. The growth rate $\sigma_{r}$ increases gradually with increasing $\lambda$. The eigenvalue for the axisymmetric case $\lambda=1$ cannot be obtained due to convergence difficulty. However, the linear stability of the axisymmetric vortex will be guaranteed as discussed in the Appendix 2.B. This result is again consistent qualitatively with the result of the simulation shown in Fig. 2.4.

Figure 2.16 shows $\sigma_{r}$ as a function of $F$ for the basic cyclonic vortices with $\eta_{\max }=0.9$ and $\lambda=1.5$. The growth rate $\sigma_{r}$ decreases almost linearly with increasing $F$, and the disturbance is stabilized when $F>F_{l c}$ $\left(1<F_{l c}<2\right)$. This result is partly consistent with the result of the simulation, where the elongation rate decreases with increasing $F$. However, the initial elongation takes place in the simulation for all $F$ explored not only when $\lambda=2.5$ as shown in Fig. 2.5, but also when $\lambda=1.5$ (not shown). This fact is not surprising, since the elongation is not always associated with instability, but occurs when the kinematic condition for the elongation is met. 


\subsubsection{Modal structure}

Next we discuss the properties of the modal structure and the phase propagation of the unstable disturbance. Figure 2.17 shows the schematic picture of the relation between the basic and disturbance fields. Figure 2.18 shows the structure of the disturbance potential vorticity for the basic cyclonic vortex with $\eta_{\max }=0.9$ or $0.4, \lambda=1.5$ and $F=0.25$. Since the eigenvalues are complex, appropriate two phases with a phase difference of $90^{\circ}$ are chosen. The disturbance has a quadrupole structure corresponding to the azimuthal wavenumber 2 . The phase propagates in the counter-clockwise direction (from the left to right panels in Fig. 2.18) due to the advection by the basic flow. In addition, the pattern oscillates in the radial direction. Since the basic flow is elliptical, the anomalies in the second and fourth quadrants (shaded regions in Fig. 2.17) are advected in the outward radial direction where the gradient of the basic potential vorticity has a positive azimuthal component. Thus the advected anomalies must be pushed back in the inward direction. On the other hand, the anomalies in the first and third quadrants (non-shaded regions in Fig. 2.17) are advected in the inward radial direction where the gradient of the basic potential vorticity has a negative azimuthal component. Thus the advected anomalies must be pushed back in the outward direction. Note that generally due to the conservation of potential vorticity, the phase of a disturbance propagates in the leftward direction when facing a gradient of the basic potential vorticity.

The behavior of the cyclonic elliptical vortex disturbed by the above unstable mode is qualitatively similar to the motion of a $q$ contour surrounding a center discussed in $\S 2.4 .4$. When the phase of the disturbance is such that the cyclonic anomalies are in the second and fourth quadrants and anticyclonic anomalies are in the first and third quadrants as shown in the left panel of Fig. 2.18, the disturbed vortex, the major axis 
of which lies in the second and fourth quadrants, is elongating along the major axis by the phase shown in the right panel of Fig. 2.18. On the other hand, when the phase is opposite, the disturbed vortex, the major axis of which lies in the first and third quadrants, is shrinking along the major axis. The above behavior of the disturbed vortex is again consistent with the axisymmetrization/elongation principle. Actually, the evolution of $\lambda_{q}$ and $\theta_{d}$ of the disturbed vortex (not shown) is qualitatively similar to that in Fig. 2.2(b), if the initial phase of the disturbance is set at the configuration for the elongation.

When $\eta_{\max }$ is considerably large as in the basic cyclone with $\eta_{\max }=0.9$ shown in Figs. 2.18(a) and 2.19(a), the disturbance is well localized inside the core of the basic vortex. Since the spatial variation of the basic depth is considerably large inside the core, a divergent motion predominates here as pointed out in $\S 2.4 .3$ (notice Fig. 2.19(a)). The horizontal scale of the disturbance increases with decreasing $\eta_{\max }$. Figures 2.18(b) and 2.19 (b) show the modal structures when $\eta_{\max }=0.4$. In this case, the distribution of the disturbance spreads over the periphery region of the basic vortex, and a divergent motion is less active inside the core. The horizontal scale of the disturbance also increases with increasing $F$ (not shown).

\subsubsection{Energetics}

According to Ripa (1983), the energy equation of the disturbance field is expressed as

$$
\varepsilon \frac{d}{d t} \int e d x d y=\int \gamma d x d y
$$

where $e$ is the disturbance energy per unit area and $\gamma$ is the energy transfer rate per unit area from the basic to disturbance field. These are 
defined as

$$
\begin{aligned}
& e=\frac{1}{2} H \boldsymbol{u} \cdot \boldsymbol{u}+\boldsymbol{U} \cdot(h \boldsymbol{u})+\frac{1}{2} F \eta^{2}, \\
& \gamma=(q \boldsymbol{u}) \times H^{2} \boldsymbol{U} .
\end{aligned}
$$

The disturbance energy increases when the flux $q \boldsymbol{u}$ of the disturbance potential vorticity has a negative angle with respect to the basic velocity $\boldsymbol{U}$ (i.e., $\gamma>0$ ) on average over the domain. Figure 2.20 shows the distribution of $\gamma$ averaged over one period, $2 \pi / \sigma_{i}$, where $\sigma_{i}=\operatorname{Im} \sigma$. The transfer rate is positive in the second and fourth quadrants, and negative in the first and third quadrants. Namely, the disturbance grows when advected by the radial component of the basic flow in the direction opposite to the radial gradient of the basic potential vorticity as implied by (2.B8). The radial oscillation seems to enhance the gain of energy in the second and fourth quadrants, and to reduce its loss in the first and third quadrants. Note the difference of the amplitudes of the divergence anomalies between in the shaded regions and in the non-shaded regions shown in the left panels of Fig. 2.19.

Finally we note the two points. First, in the framework of a linear theory, the initial phase of a disturbance is arbitrary, that is, the linear theory cannot predict whether the elongation or axisymmetrization will occur at an initial stage. In the actual numerical simulation, the initial phase of the disturbance is determined during an initial adjustment process which comes from that the initial conditions are not steady solutions. As concerns this, the relation of $\lambda_{q}$ and $\lambda_{s}$ can qualitatively predict the initial phase as discussed in $\S 2.4$.4. Second, the linear evolution passes through the two stages, that is, the stages of the elongation and axisymmetrization, periodically. In the numerical simulation (in the nonlinear 
evolution), however, the flow evolution does not always pass through both stages. The axisymmetrization (in the regime A or at the later stage of the regime $\mathrm{AE}$ ) accompanies the forward transfer of enstrophy associated the generation of thin filaments. Thus this process is irreversible, and the successive stage of the elongation is never realized in the nonlinear evolution. The elongation causes the split-up of a vortex core when $\eta_{\max }$ is large enough, thus this process is also irreversible. Only in the regime $\mathrm{AE}$, the initial elongation does not accompany the change of the type of the stagnation point, thus this elongation ceases at some stage and the nonlinear evolution enters the stage of the axisymmetrization as in the linear evolution.

\subsection{Summary and discussion}

The free evolution of an isolated elliptical vortex is investigated numerically in a rotating shallow-water system. In contrast with the quasigeostrophic cases, it is found that the evolution of cyclonic elliptical vortices is classified into three regimes ( $\mathrm{A}, \mathrm{AE}$, and $\mathrm{SE}$ ) depending on the maximum surface displacement $\eta_{\max }$ with the rotational Froude number $F$ fixed. The core of a vortex approaches axisymmetry (the regime A) when $\eta_{\max }$ is below the first critical value, while contours of potential vorticity $q$ of the vortex core are elongated when $\eta_{\max }$ is above this value. Furthermore, the core of a vortex splits up into two as a consequence of elongation (the regime SE) when $\eta_{\max }$ is above the second critical value. On the other hand, the elongation ceases at some stage and the axisymmetrization prevails finally (the regime $\mathrm{AE}$ ) when $\eta_{\max }$ is between the first and second critical values. Here the split-up of the vortex core is defined by the change of the sign of the Gaussian curvature of the corotating streamfunction at the center of a vortex. The first and second critical values about $\eta_{\max }$ increase with increasing $F$, and the regime SE 
no longer occurs when $F$ exceeds some critical value. In contrast, for anticyclonic elliptical vortices the axisymmetrization always takes place irrespective of the values of $\eta_{\max }$ and $F$. The axisymmetric vortices are always stable irrespective of the polarity and the values of $\eta_{\max }$ and $F$ explored, as opposed to the result of Cushman-Roisin and Tang (1990).

A kinematic condition for the elongation/axisymmetrization is derived by considering the motion of a $q$ contour surrounding the stagnation point (whose type is approximately a center) at the center of a vortex. According to this, the geometry for the elongation, where the $q$ distribution lags behind the distribution of transport streamfunction, is realized when the aspect ratio of the $q$ distribution inside the vortex core is smaller than that defined by the strain and vorticity at the center. Furthermore, it is found that the split-up of the core always occurs when the stagnation point changes into a saddle at an early stage. The time lag of this change and the split-up suggests an important role of an ageostrophic motion associated with considerably thin fluid depth inside the core. The appearance of a saddle inside the core is at least a necessary condition for the split-up.

The linear stability analysis shows that not only cyclonic but also anticyclonic elliptical vortices are unstable. However, the growth rate of the disturbance for anticyclones is much smaller than that for cyclones. The growth rate for cyclones increases almost linearly as $\eta_{\max }$ is increased with $F$ fixed, while decreases as $F$ is increased with $\eta_{\max }$ fixed. These dependences on $\eta_{\max }$ and $F$ are consistent with the results of the above numerical simulations. The unstable disturbance has a quadrupole structure corresponding to the azimuthal wavenumber 2 , and the disturbed elliptical vortex undergoes an elongating-shrinking motion of $q$ contours with a tilting motion of their principal axes. This phase change is consistent with the kinematics of the elongation and axisymmetrization. 
The above results have relevance to those of the turbulent simulations described in Part 1. The elongation and break-up of cyclones in the turbulent evolution is attributed to the inherent instability of an isolated cyclonic elliptical vortex, when the vertical displacement is large. On the other hand, a cyclonic vortex with small displacement or an anticyclonic vortex with arbitrary displacement are much less unstable, and the growth of the unstable disturbance is masked by the tendency for the axisymmetrization.

In the frontal geostrophic regime, the origin of the above asymmetry between cyclones and anticyclones is expressed in the form of the cubic nonlinearity. However, just in the parameter regime where the geostrophic approximation is invalid, the remarkable phenomenon, i.e., the split-up of a vortex occurs. On the other hand, the split-up is suppressed in the frontal geostrophic regime. Thus a simplified equation such as the frontal geostrophic equation does not always provide a good description of vortices with strong vertical displacements which are relevant to oceanic mesoscale or submesoscale vortices.

\section{Appendix 2.A. $q$-ellipse and $\Psi$-ellipse}

We define the core region of a vortex by the region where gradients of the potential vorticity $q$ will not tend to grow as discussed in $\S 1.5$. Thus we again define the core region by the region with $\Lambda<0$ where vorticity exceeds strain. Here $\Lambda$ is given by (1.20b). We define the boundary of the core by an appropriate $q$ contour inside this region. Here we adopt the contour which is tangent to the $\Lambda$ contour with $\Lambda=0.1 \cdot \Lambda_{0}$ on the major axis at $t=0$, where $\Lambda_{0}$ is the value of $\Lambda$ at the center of a vortex.

We introduce the $q$-ellipse by an ellipse approximate to the $q$ contour of the core. We define the factors to quantify the shape of the region 
enclosed by a $q$ contour according to McWilliams (1990) as follows:

$$
\begin{aligned}
\delta x_{i} & =\frac{\int x_{i}^{\prime} d x_{1} d x_{2}}{\int d x_{1} d x_{2}} \\
M_{i j} & =\frac{\int\left(x_{i}^{\prime}-\delta x_{i}\right)\left(x_{j}^{\prime}-\delta x_{j}\right) d x_{1} d x_{2}}{\int d x_{1} d x_{2}},
\end{aligned}
$$

where $\left(x_{1}^{\prime}, x_{2}^{\prime}\right)$ is the spatial coordinate relative to the extremum of the $q$ distribution inside the region, $\left(\delta x_{1}, \delta x_{2}\right)$ is the first-order moment and $M_{i j}$ is the second-order moment of the region. A closed $q$ contour is then represented approximately by an ellipse with a major axis $a=2 \sqrt{M^{(1)}}$ and a minor axis $b=2 \sqrt{M^{(2)}}$, where $M^{(1)}$ and $M^{(2)}\left(M^{(1)}>M^{(2)}\right)$ are the eigenvalues of the matrix $M_{i j}$. The aspect ratio $\lambda_{q}$ of the $q$-ellipse is defined by $\lambda_{q}=a / b$.

As long as there exists only one extremum inside the core region, the extremum position coincides with the origin due to the spatial symmetry of the initial conditions. Thus the first-order moment is exactly zero. But if there appear two extrema, the first-order moment is no longer zero. In this situation, if the $q$ contour of the core does not break up, that is, if the contour encloses the two extrema at $\left(x_{e}, y_{e}\right)$ and $\left(-x_{e},-y_{e}\right)$, then the centroid position $\left(x_{e}+\delta x_{1}, y_{e}+\delta x_{2}\right)$ of the region coincides with the origin. Here we call such a state the local split-up of the vortex core. If the contour breaks up into two contours each of which encloses each extremum position, the core is said to be in the state of the complete split-up. In this state, the first-order moment gives a slight correction to the centroid position due to a slight deformation from an elliptical shape. The angle $\theta_{q}$ is defined by the orientation of the major axis for the single vortex state and the local split-up state, and by the orientation of the axis which connects the centroid positions of each vortex for the complete split-up state. 
We define the $\Psi$-ellipse by the transport streamfunction $\Psi$ in the same way as the $q$-ellipse. Here the $\Psi$ contour of the core is defined by the contour which is tangent to the $q$ contour of the core on the major axis at $t=0$. The orientation $\theta_{\Psi}$ of the $\Psi$-ellipse is also determined as in $\theta_{q}$.

The angular velocity $\Omega$ of the revolution is defined by $\Omega=\frac{d \theta_{\Psi}}{d t}$. Although $\frac{d \theta_{\Psi}}{d t}$ changes as the axisymmetrization or elongation develops, in the initial phase just after the adjustment process, $\frac{d \theta_{\Psi}}{d t}$ is approximately constant. Here $\Omega$ is evaluated by the linear approximation to the $t-\theta_{\Psi}$ curve using the least-squares method in the initial phase, and by the differentiation of this curve using the third-order cubic spline function in the later phase.

We display two $q$ contours by solid bold lines on the contour plots in Figs. 2.1, 2.10, 2.18-2.20, and 2.C. The inner contour, which lies in the core region, is the contour of the $q$-ellipse defined above. The outer contour, which lies in the periphery region, is the contour with $q=1.05$ for a cyclonic vortex and with $q=0.95$ for an anticyclonic vortex. In Fig. 2.11 , the $q$ contour of the core alone is displayed.

\section{Appendix 2.B. Linear stability of an axisymmetric vortex}

According to Ripa's integral theorem (Ripa 1983), we discuss the linear stability of an axisymmetric vortex.

First we extend his discussion to an axisymmetric flow in terms of the conservative quantities on an $f$-plane, the total energy and the angular momentum. We consider an axisymmetric, steady flow in gradient-wind balance as

$$
\varepsilon \frac{V^{2}}{r}+V=\frac{1}{\varepsilon F} \frac{d H}{d r}
$$

where $V(r)$ is the azimuthal velocity and $H(r)$ is the fluid depth. The 
inviscid, linearized shallow-water equations around the above basic state are expressed in the polar coordinate $(r, \theta)$ as

$$
\begin{aligned}
& \varepsilon D_{t} u-\left(\varepsilon \frac{2 V}{r}+1\right) v+\frac{1}{\varepsilon F} \partial_{r} h=0, \\
& \varepsilon D_{t} v+H Q u+\frac{1}{\varepsilon F} \frac{1}{r} \partial_{\theta} h=0, \\
& D_{t} h+\frac{1}{r} \partial_{r}(r H u)+\frac{1}{r} H \partial_{\theta} v=0 .
\end{aligned}
$$

Here $(u, v)$ and $h$ are the velocity and fluid depth of the disturbance field, respectively, $D_{t}=\partial_{t}+\frac{V}{r} \partial_{\theta}$ is the linearized Lagrangian derivative, and $Q=\frac{1}{H}\left[\varepsilon \frac{1}{r} \partial_{r}(r V)+1\right]$ is the potential vorticity of the basic field. The potential vorticity $q$ of the disturbance field is defined as

$$
q=\frac{1}{H}\left[\varepsilon\left(\frac{1}{r} \partial_{r}(r v)-\frac{1}{r} \partial_{\theta} u\right)-Q h\right]
$$

which yields

$$
D_{t} q+Q_{r} u=0
$$

where $Q_{r}=\partial_{r} Q$. The total energy density $e$ and the angular momentum density $m$ of the disturbance field are defined as

$$
\begin{aligned}
& e=\frac{1}{2} H\left(u^{2}+v^{2}\right)+V h v+\frac{1}{2 \varepsilon^{2} F} h^{2}, \\
& m=r h v .
\end{aligned}
$$

These quantities yield

$$
\begin{aligned}
& \varepsilon \partial_{t}\langle e\rangle+\varepsilon \frac{1}{r} \partial_{r}\left(r V H\langle u v\rangle+\varepsilon^{-2} F^{-1} r H\langle h u\rangle\right)-V H^{2}\langle q u\rangle=0,(2 . \mathrm{B} 4 \mathrm{a}) \\
& \varepsilon \partial_{t}\langle m\rangle+\varepsilon \frac{1}{r} \partial_{r}\left(r^{2} H\langle u v\rangle\right)-r H^{2}\langle q u\rangle=0
\end{aligned}
$$

where $\langle\cdots\rangle$ denotes the azimuthal average. We introduce the displacement $\xi$ of a fluid particle in the radial direction. The linearized form of 
the relation between $\xi$ and the Eulerian velocity $u$ gives

$$
D_{t} \xi=u
$$

In terms of this, (2.B3) is rewritten as

$$
D_{t}\left(q+Q_{r} \xi\right)=0
$$

which has the particular solution of the form

$$
q=-Q_{r} \xi
$$

Thus

$$
\langle q u\rangle=-\frac{1}{2} Q_{r} \partial_{t}\left\langle\xi^{2}\right\rangle .
$$

This implies that the radial flux $\langle q u\rangle$ of the potential vorticity must be in the direction opposite to the basic potential vorticity gradient $Q_{r}$ in order that a disturbance grows (i.e., $\partial_{t}\left\langle\xi^{2}\right\rangle>0$ ). In terms of (2.B8), and after integrating over $0 \leq r<\infty,(2 . \mathrm{B} 4 \mathrm{a})$ and $(2 . \mathrm{B} 4 \mathrm{~b})$ are reduced to

$$
\begin{aligned}
& \partial_{t} \int_{0}^{\infty} r d r\left\langle\varepsilon e+\frac{1}{2} V H^{2} Q_{r} \xi^{2}\right\rangle=0, \\
& \partial_{t} \int_{0}^{\infty} r d r\left\langle\varepsilon m+\frac{1}{2} r H^{2} Q_{r} \xi^{2}\right\rangle=0 .
\end{aligned}
$$

The combination of the above equations satisfies

$$
\partial_{t} \int_{0}^{\infty} r d r\left\langle\varepsilon(e-\alpha m)+\frac{1}{2} H^{2} \xi^{2}(V-r \alpha) Q_{r}\right\rangle=0,
$$

where $\alpha$ is any constant. If there exists some constant $\alpha$ which satisfies

$$
[V(r)-r \alpha] \cdot Q_{r}(r) \geq 0 \quad \text { and } \quad H(r) \geq \varepsilon^{2} F[V(r)-r \alpha]^{2}
$$

for all $r$, the integrand of (2.B10) is positive definite. This implies that the increase of $\langle e-\alpha m\rangle$ contradicts the increase of $\left\langle\xi^{2}\right\rangle$. Thus the basic state must be stable. The above conditions are sufficient conditions for the stability of a basic state to infinitesimal disturbances. 
Next we apply the above theorem. In the present initial conditions, (2.1) with $\lambda=1$ and (2.B1), the basic fields are given by

$$
\begin{aligned}
& V(r)=s r \exp \left(-\frac{r^{2}}{2}\right) \\
& H(r)=1-\varepsilon F \exp \left(-\frac{r^{2}}{2}\right)\left[s+\frac{1}{2} \varepsilon \exp \left(-\frac{r^{2}}{2}\right)\right] .
\end{aligned}
$$

There exists a point $r=r_{*}$ at which $Q_{r}(r)$ changes its sign. For cyclonic vortices $(s=1), Q_{r}(r)$ is negative for $r<r_{*}$ and positive for $r>r_{*}$. On the other hand, $V(r) / r$ is a monotonically decreasing function. In such profiles, we cannot easily find an appropriate constant $\alpha$ which satisfies (2.B11) for all $r$. Here we note that the point $r=r_{*} \sim 2$ is located far outside the vortex core. Thus we may consider only the core region of the basic vortex. For an axisymmetric case, $\Lambda$ defined by (1.20b) is reduced to

$$
\Lambda(r)=-\frac{V}{r} \cdot \frac{d V}{d r}
$$

and for the present basic state (2.B12a),

$$
\Lambda(r)=-\left(1-r^{2}\right) e^{-r^{2}}
$$

This shows that the vortex core (with $\Lambda<0$ ) is distributed inside the region $r<1$, where $s Q_{r}(r)$ is negative definite. If we choose $\alpha=V(r) /\left.r\right|_{r=0}$, the conditions (2.B11) are satisfied inside the core for both polarities $s= \pm 1$ and for all values of $\eta_{\max }$ and $F$ explored in the numerical simulations. Therefore we may conclude that the present axisymmetric vortices will be stable to infinitesimal disturbances. This is consistent with the results of the simulation as described in $§ 2.3 .3$. 


\section{Appendix 2.C. Mechanism for the axisymmetrization}

We briefly review the mechanism for the axisymmetrization according to Melander et al. (1987), and then examine its validity in the present shallow-water cases.

The contours of the potential vorticity $q$ at the periphery of an elliptical vortex form spiral filaments due to the presence of the saddle points as explained in $\S 2.4 .3$. The spiral $q$ distribution induces the lagged $\Psi$ distribution behind the $q$ distribution as a consequence of a smoothing effect. This geometry drives the axisymmetrization as explained in $\S 2.4 .1$. In the quasi-geostrophic case, the smoothing effect is implied by the relation

$$
\psi(x, y)=-\frac{1}{2 \pi} \int K_{0}(\sqrt{F} r) q\left(x^{\prime}, y^{\prime}\right) d x^{\prime} d y^{\prime},
$$

which is the inversion of (1.7). Here $K_{0}$ is the modified Bessel function of the zeroth order, and $r=\sqrt{\left(x-x^{\prime}\right)^{2}+\left(y-y^{\prime}\right)^{2}}$. The above integral relation implies that the sharp filamentary structure of the $q$ distribution is smoothed in the $\psi$ distribution when $F$ is not so large. The axisymmetrization is possible due to the inevitable presence of saddle points inside the $q$ distribution, when the initial $q$ distribution is smooth as in the present case. This is quite contrast to the case with a piecewiseconstant $q$ distribution, where the $q$ distribution is initially confined to the inner separatrices (Polvani 1989).

In the shallow-water case the streamfunction $\psi$ is replaced by the transport streamfunction $\Psi$. In order to examine influences of the global $q$ distribution on the $\Psi$ distribution, we show the evolution of the $\Psi$ field and the two $q$ contours for the cyclonic elliptical vortices in Fig. 2.C. In the case $\eta_{\max }=0.09$, as the filaments develop at the periphery, the $\Psi$ distribution actually lags behind the $q$ distribution in the core region. This result is consistent with the above proposition, since the quasi-geostrophic approximation holds well in this case. On the other 
hand, in the case $\eta_{\max }=0.9$, the $q$ distribution lags behind the $\Psi$ distribution in the core region. This fact is surprising since the spiral filaments developing at the periphery should induce the opposite geometry, i.e., the geometry for the axisymmetrization. Thus the above facts lead to the following suggestions. The elongation will be caused by a local dynamics inside the core region, in contrast with the axisymmetrization which is driven by the velocity field induced by the global $q$ distribution. The driving mechanism for the axisymmetrization inherently exists irrespective of the magnitude of $\eta_{\max }$. However, the elongation will suppress the axisymmetrization when $\eta_{\max }$ is large. In other words, the elongation is suppressed by the tendency for the axisymmetrization when $\eta_{\max }$ is small. 

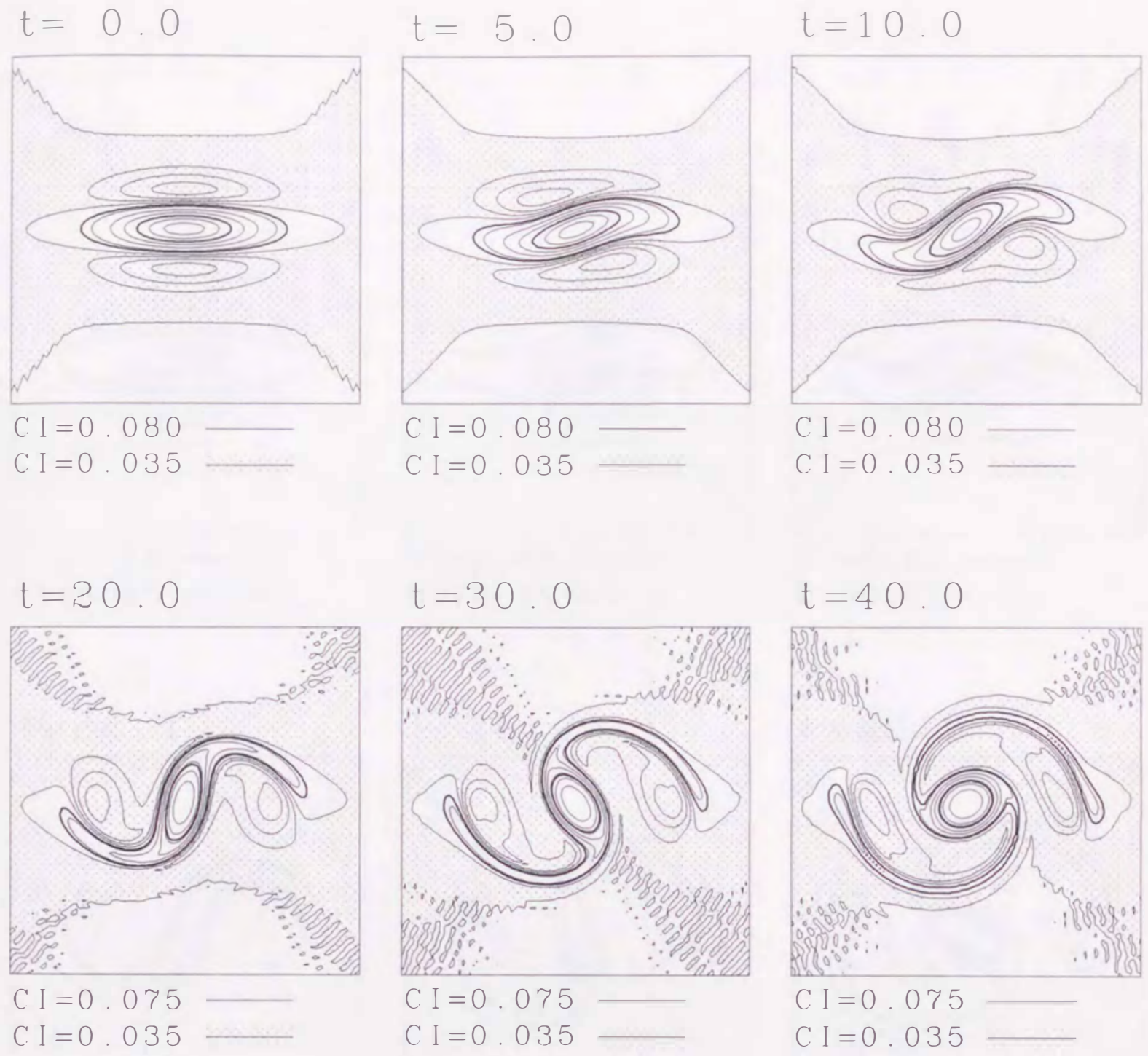

$\mathrm{C} I=0.035$

Fig. 2.1(a). Evolution of the potential vorticity $q$ for the cyclonic elliptical vortex with $\eta_{\max }=0.09, \lambda=2.5$ and $F=0.25$. The inner and outer bold lines are the contours representing the core and periphery of the vortex, respectively (See the Appendix 2.A for their definitions). The anticyclonic region $(q<1)$ is shaded. The intervals of contours are denoted by CI below each panel. Only the part $|x|,|y| \leq 8$ of the domain is shown. 


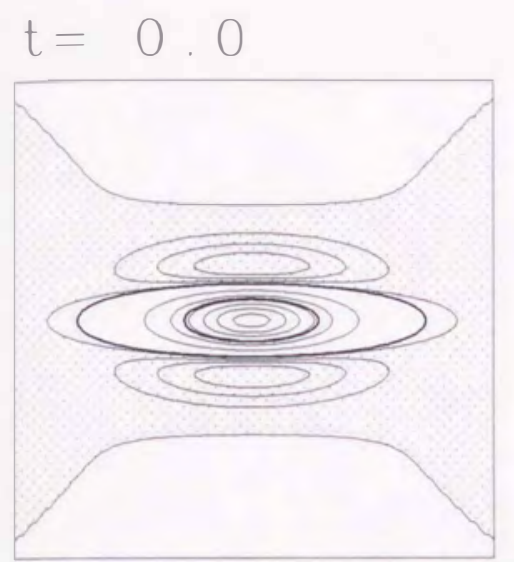

$\mathrm{C} \mathrm{I}=0.70$ C I $=0.15$

\section{$t=24$}

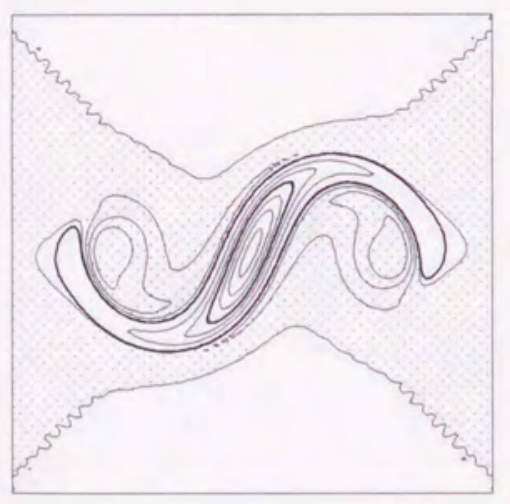

$\mathrm{C} I=0.65$

$\mathrm{C} I=0.15$

\section{$t=6 \cdot 0$}
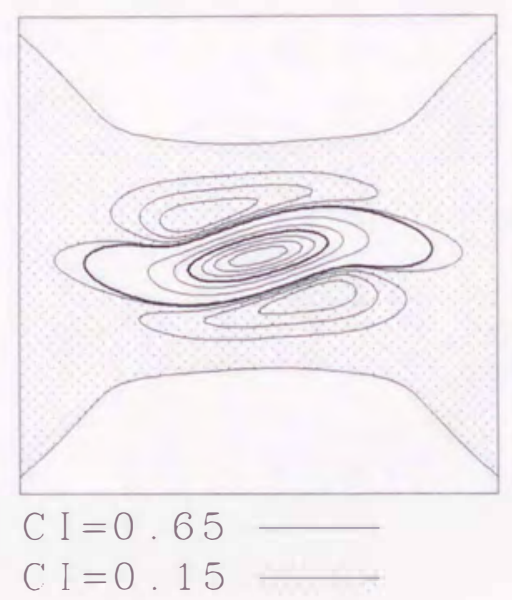

$t=12 \cdot 1$

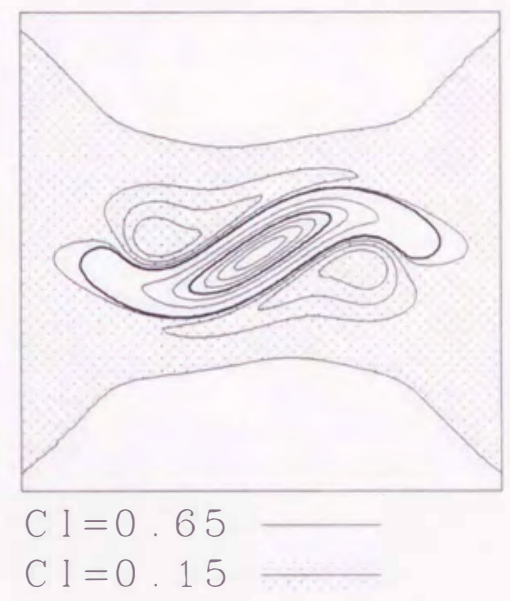

$t=36.2$

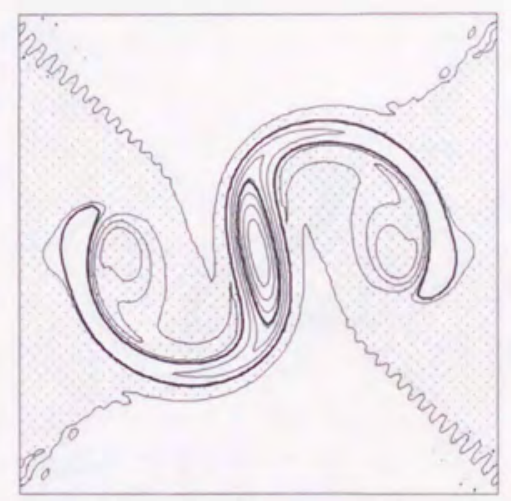

$\mathrm{CI}=0.60$
$\mathrm{CI}=0.15$

\section{$t=48.3$}

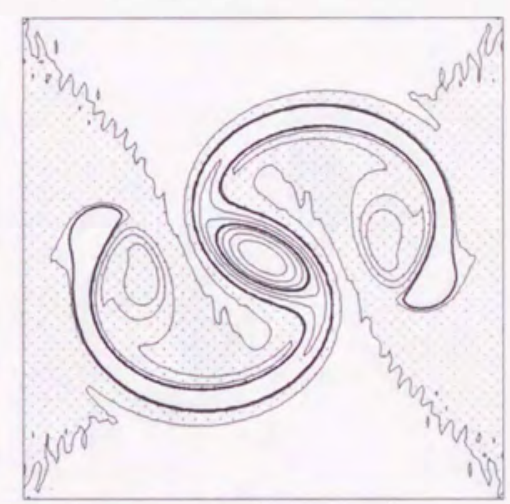

C I $=0.60$

C I $=0.15$

Fig. 2.1(b). As in Fig. 2.1(a) but with $\eta_{\max }=0.5$. 


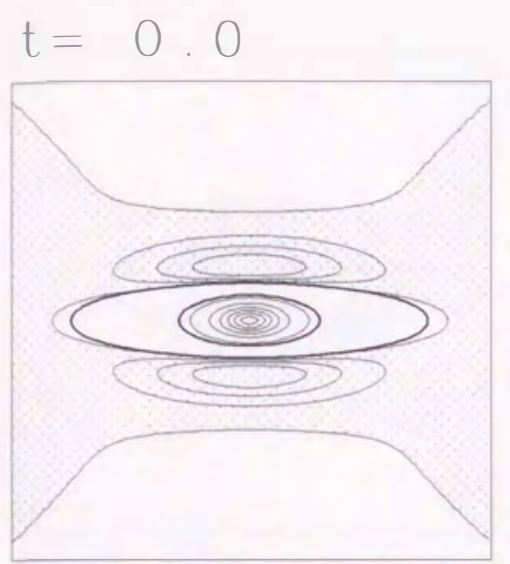

$\mathrm{C} I=5.5$ C I $=0.2$

\section{$t=19.7$}

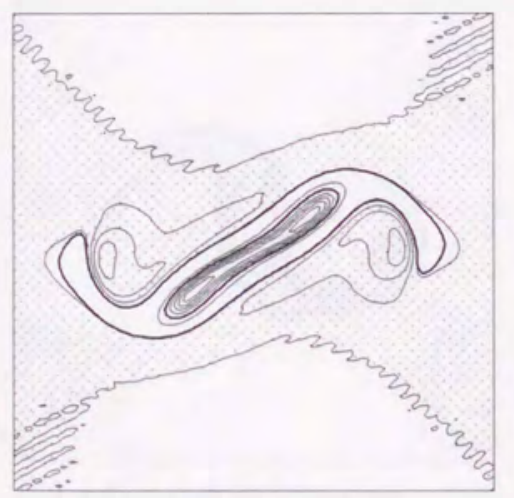

$\mathrm{C} I=2.0$

$\mathrm{C} I=0.3$
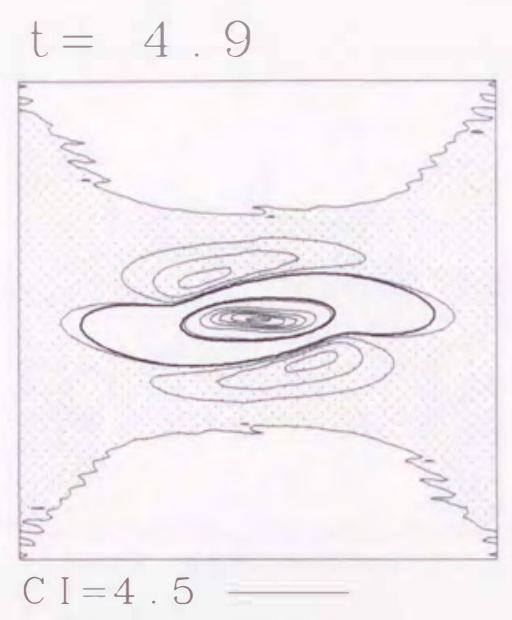

$\mathrm{C} I=0.3$

$t=29.5$

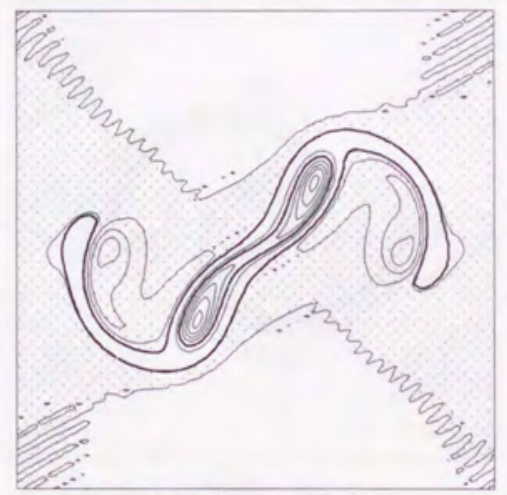

$\mathrm{CI}=2.0$

$\mathrm{C} I=0.3$

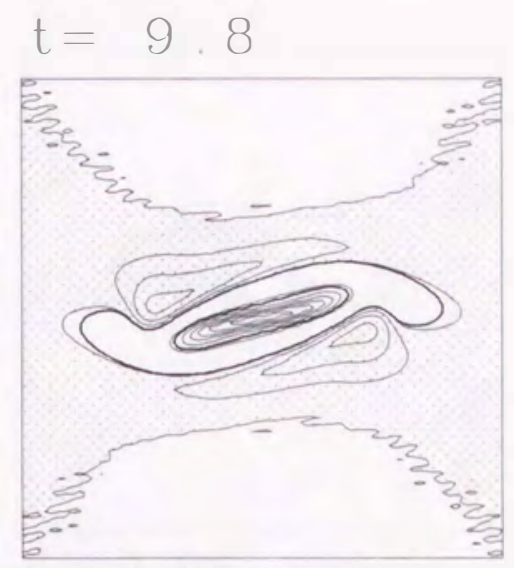

$\mathrm{C} I=3.0$

C I $=0.3$

\section{$t=39.4$}

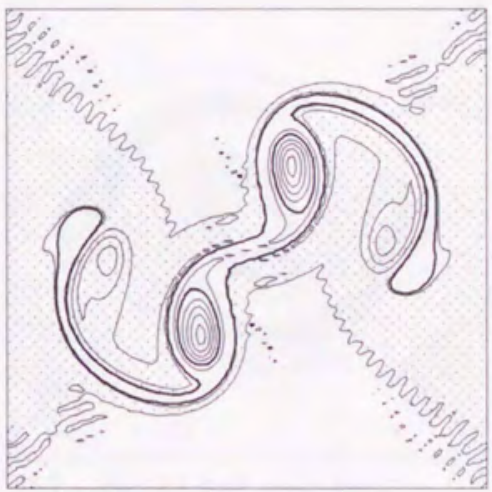

C I $=2.0$

$\mathrm{C} I=0.3$

Fig. 2.1(c). As in Fig. 2.1(a) but with $\eta_{\max }=0.9$. 


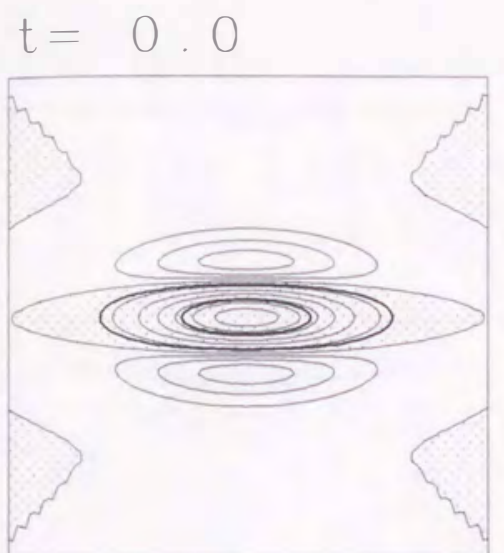

$\mathrm{CI}=0.040$

$\mathrm{CI}=0.075$

$t=20$

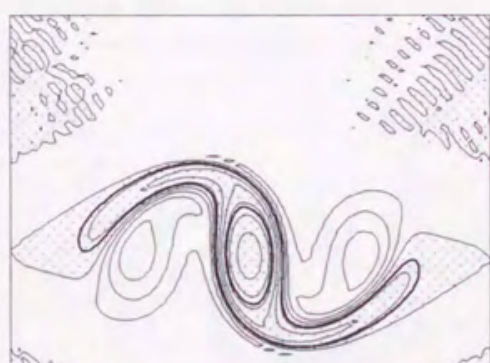

ind

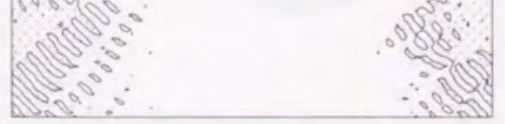

$\mathrm{CI}=0.040$

$\mathrm{CI}=0.075$ $t=5 \cdot 0$
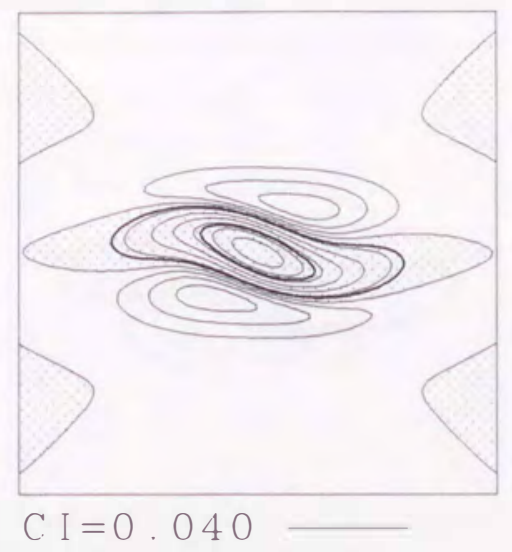

C. $I=0.075$

$$
t=30.0
$$

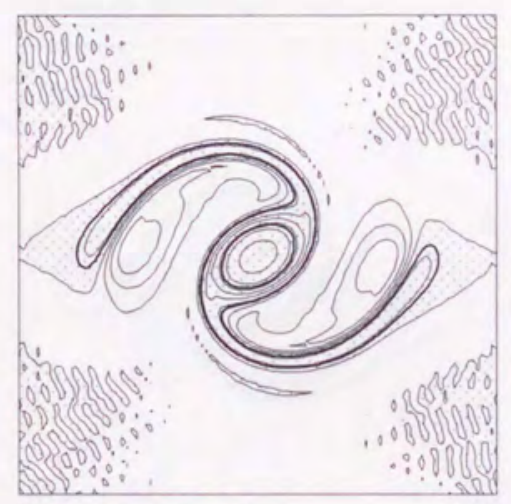

$\mathrm{C} \mathrm{I}=0.040$

C. $I=0.075$ $t=10 \cdot 0$

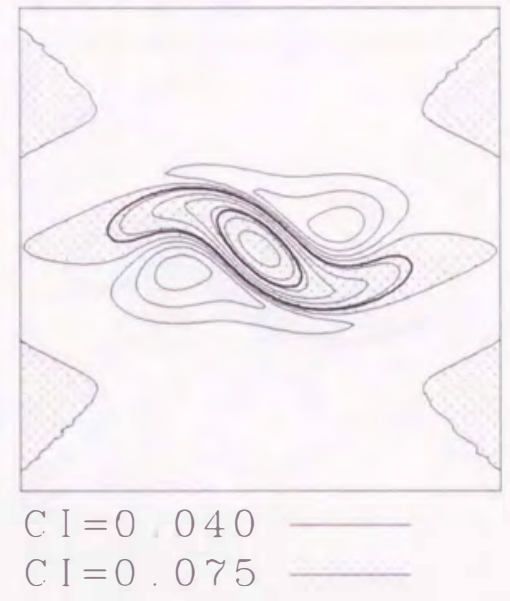

$t=40$

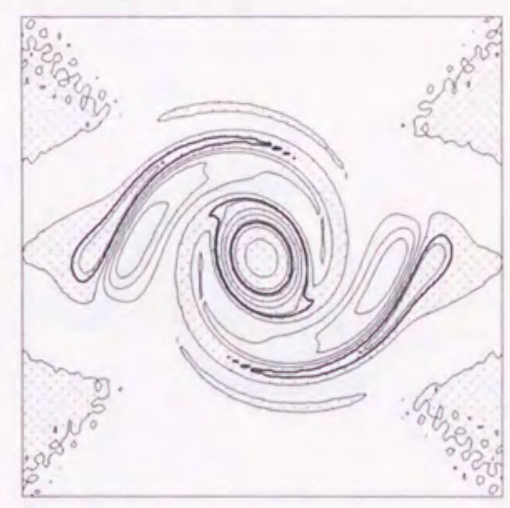

C. $I=0.040$

C. $I=0.075$

Fig. 2.1(d). As in Fig. 2.1(a), but for the anticyclonic elliptical vortex with $\eta_{\max }=0.09$. 

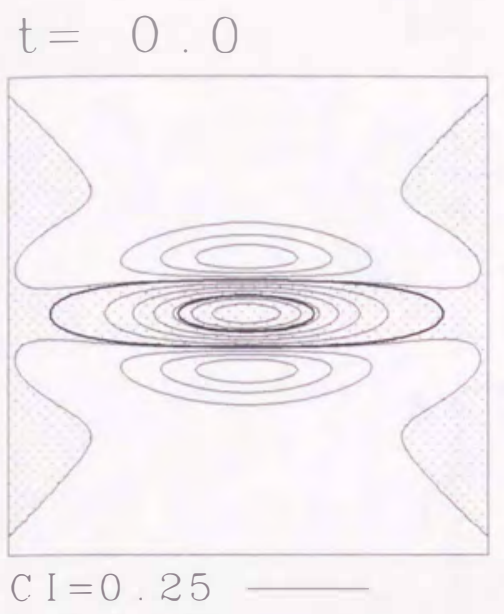

C I $=0.35$

$\mathrm{t}=19.7$

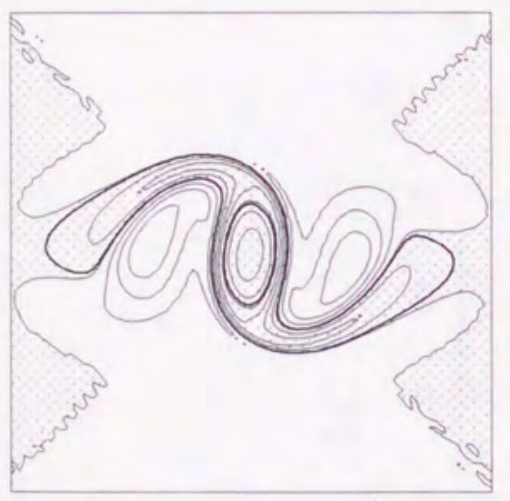

C I $=0.25$

C I $=0.35$

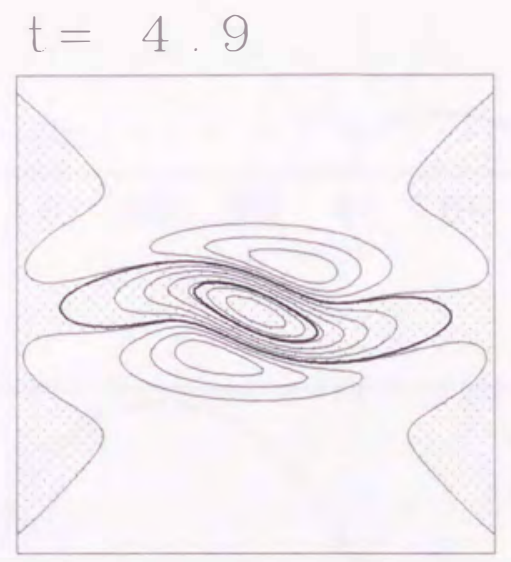

$\mathrm{C} I=0.25$

$\mathrm{C} I=0.35$

$t=29.5$

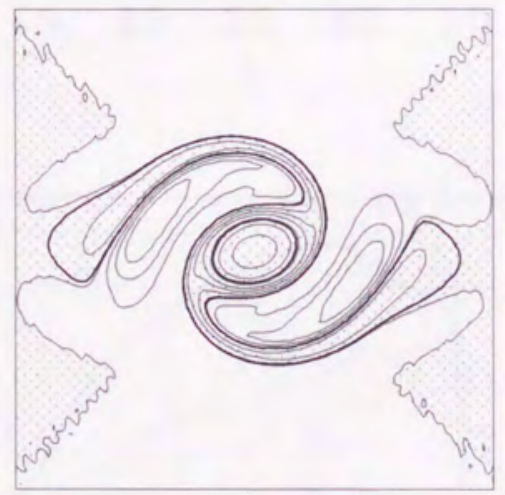

C I $=0.25$

C I $=0.35$

\section{$t=9.8$}

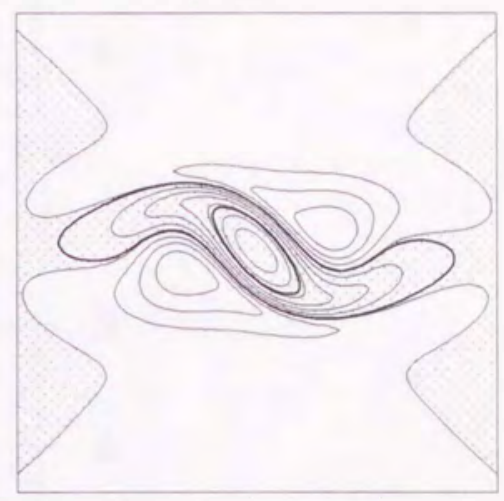

$\mathrm{C} I=0.25$

C I $=0.35$

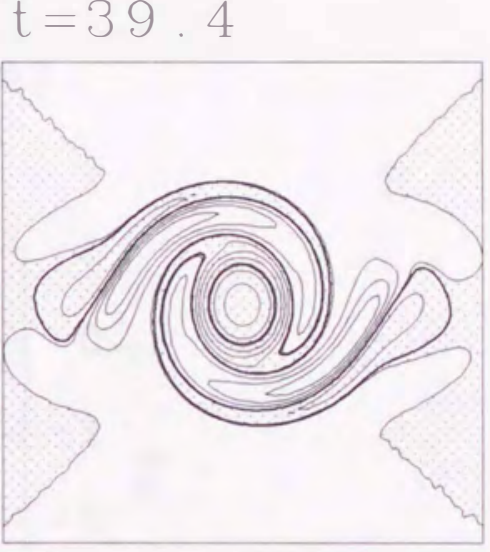

C I $=0.25$

$\mathrm{C} I=0.35$

Fig. 2.1(e). As in Fig. 2.1(d) but with $\eta_{\max }=0.34$. 

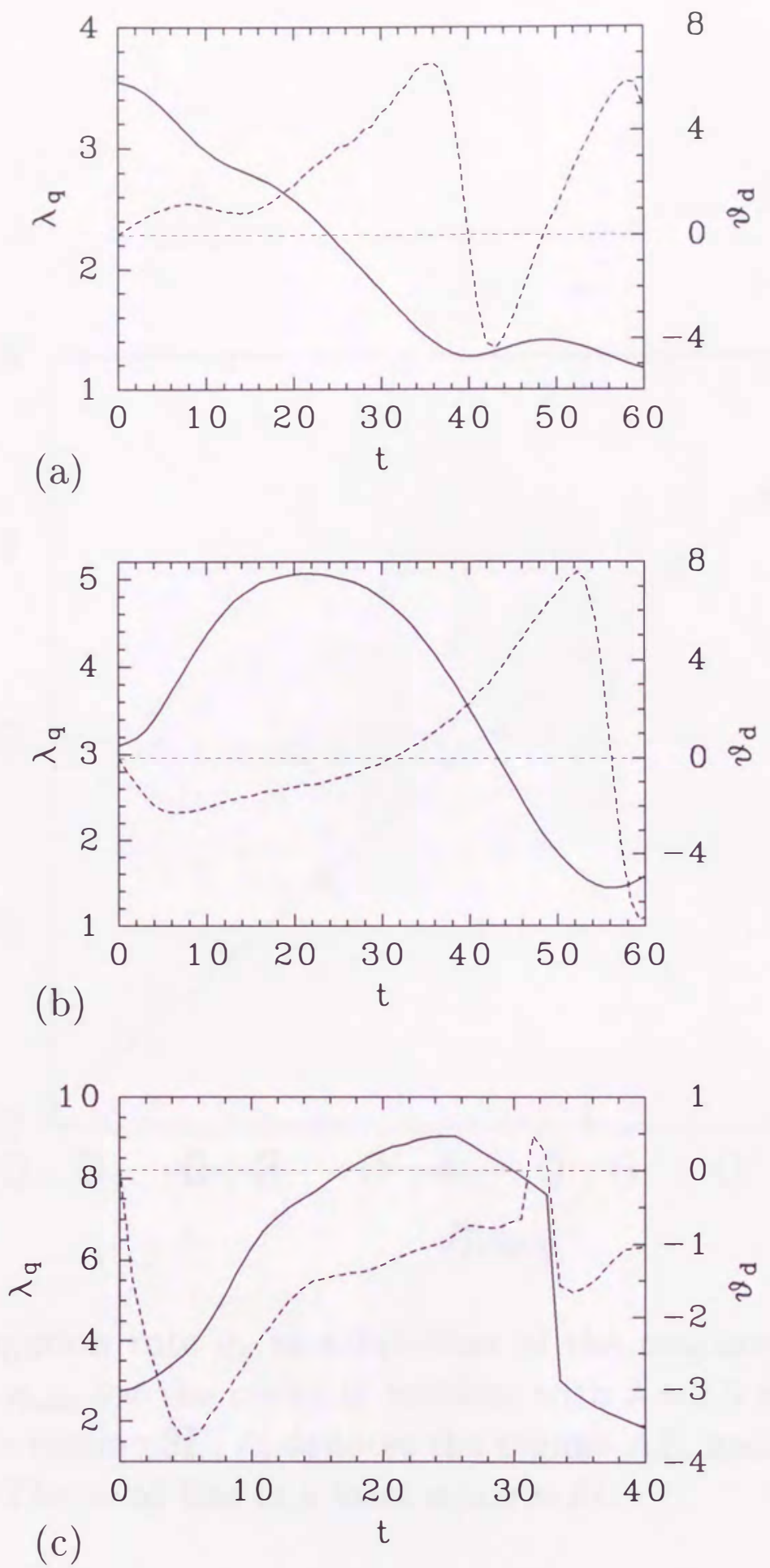

Fig. 2.2. Evolution of the aspect ratio $\lambda_{q}$ of the $q$-ellipse (solid line) and the difference angle $\theta_{d}$ (dashed line) for the cyclonic vortex with $\lambda=2.5, F=0.25$, and (a) $\eta_{\max }=0.09$; (b) $\eta_{\max }=0.5$; (c) $\eta_{\max }=0.9$. The angle $\theta_{d}$ is measured in degrees. 


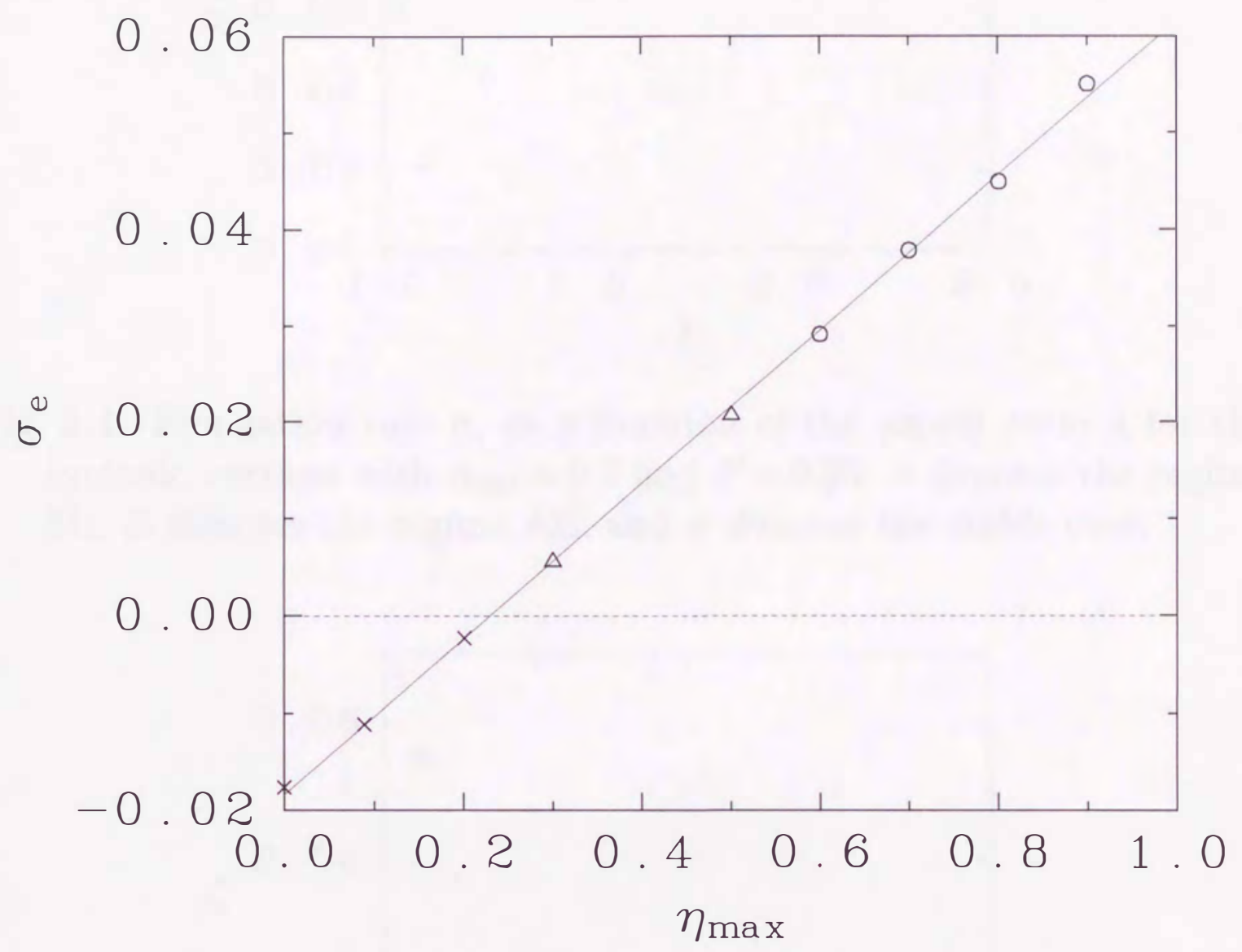

Fig. 2.3. Elongation rate $\sigma_{e}$ as a function of the maximum surface displacement $\eta_{\max }$ for the cyclonic vortices with $\lambda=2.5$ and $F=0.25$. denotes the regime $\mathrm{SE}, \triangle$ denotes the regime $\mathrm{AE}$, and $\times$ denotes the regime $\mathrm{A}$. The solid line is a least squares fit. 


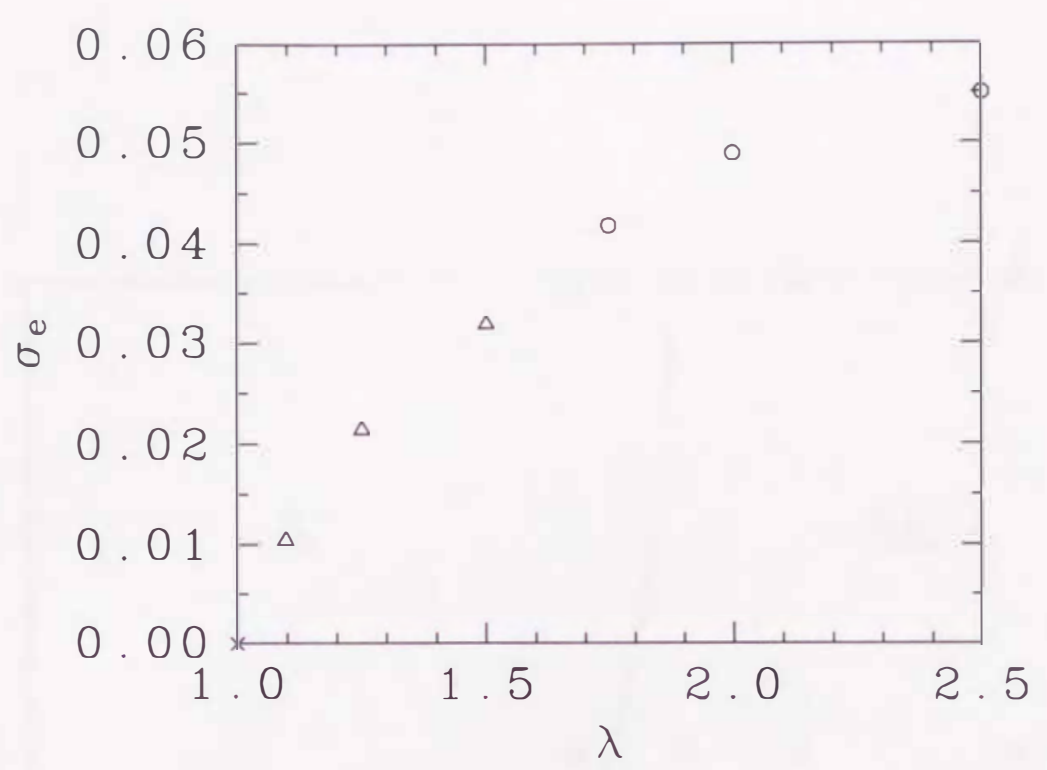

Fig. 2.4. Elongation rate $\sigma_{e}$ as a function of the aspect ratio $\lambda$ for the cyclonic vortices with $\eta_{\max }=0.9$ and $F=0.25$. $\circ$ denotes the regime $\mathrm{SE}, \triangle$ denotes the regime $\mathrm{AE}$, and $\times$ denotes the stable case.

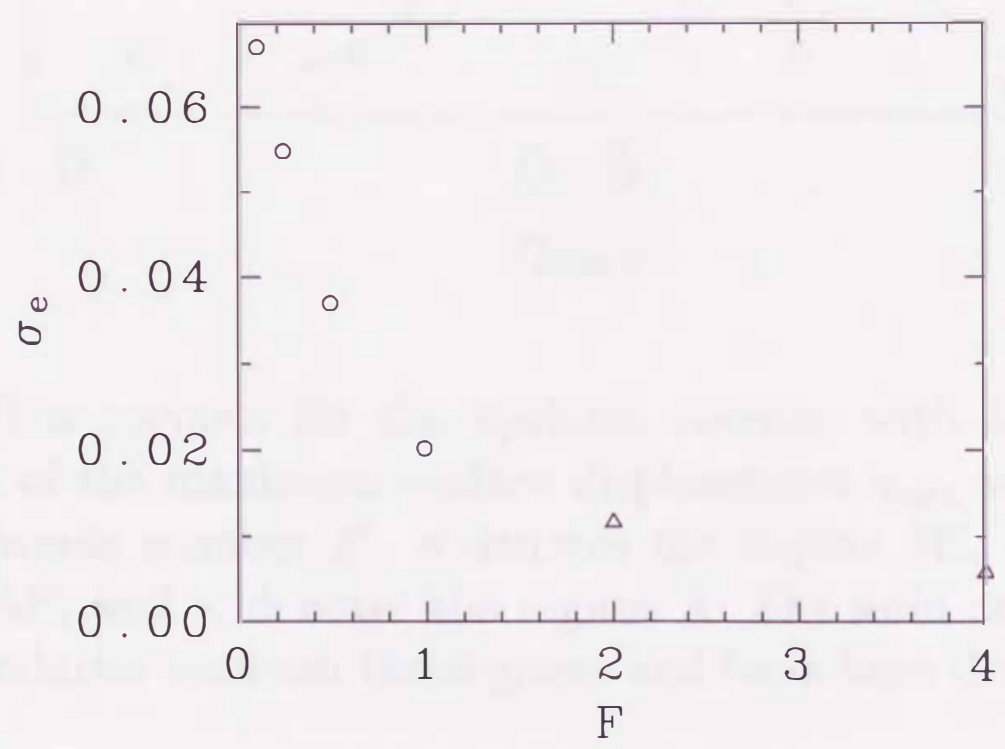

Fig. 2.5. Elongation rate $\sigma_{e}$ as a function of the rotational Froude number $F$ for the cyclonic vortices with $\eta_{\max }=0.9$ and $\lambda=2.5$. $\circ$ denotes the regime $\mathrm{SE}$ and $\triangle$ denotes the regime $\mathrm{AE}$. 


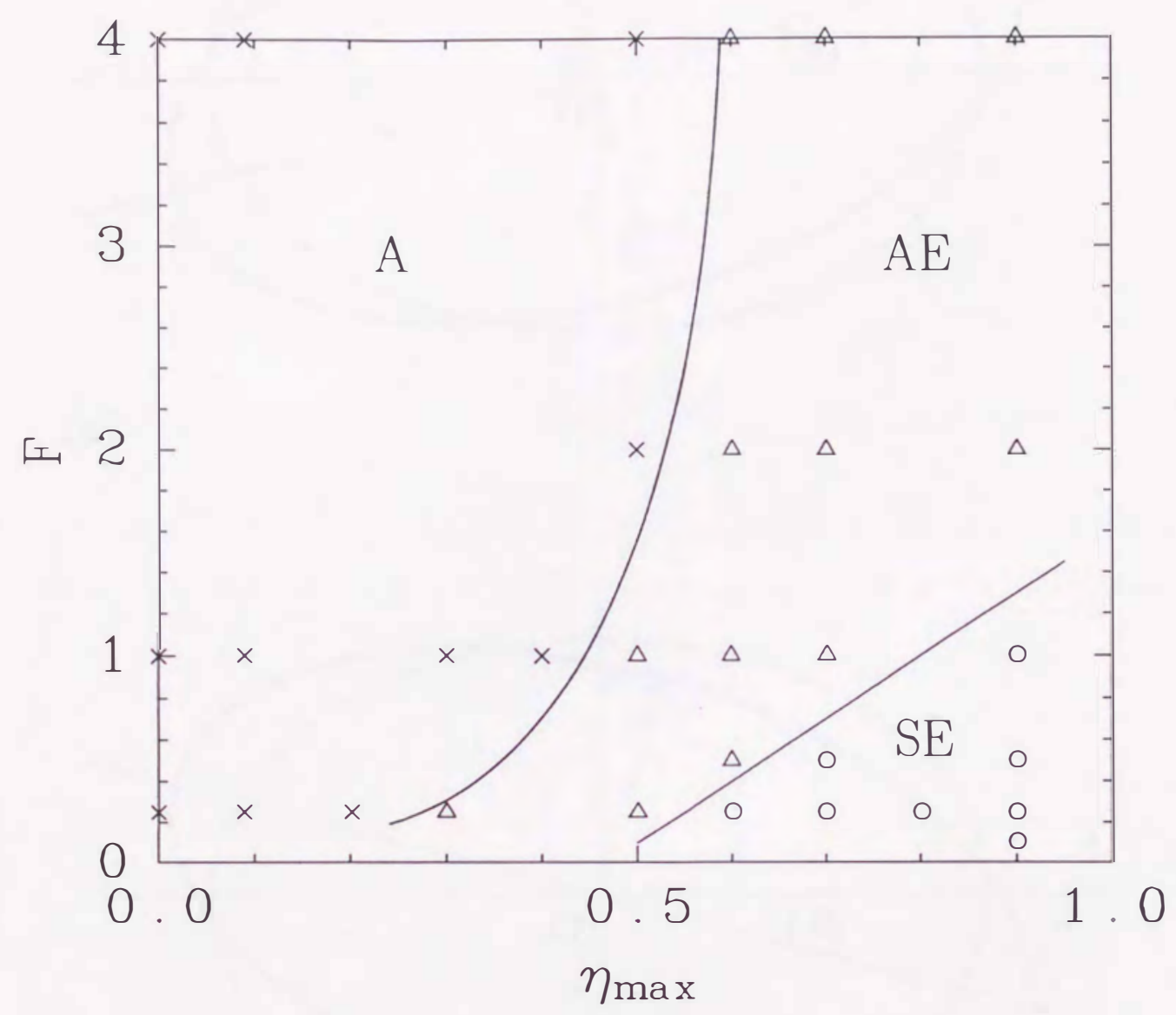

Fig. 2.6. Flow regimes for the cyclonic vortices with $\lambda=2.5$ on the diagram of the maximum surface displacement $\eta_{\max }$ versus the rotational Froude number $F$. o denotes the regime SE, $\triangle$ denotes the regime $\mathrm{AE}$, and $\times$ denotes the regime $\mathrm{A}$. The solid curves represent the boundaries between the regimes and have been drawn by hand. 


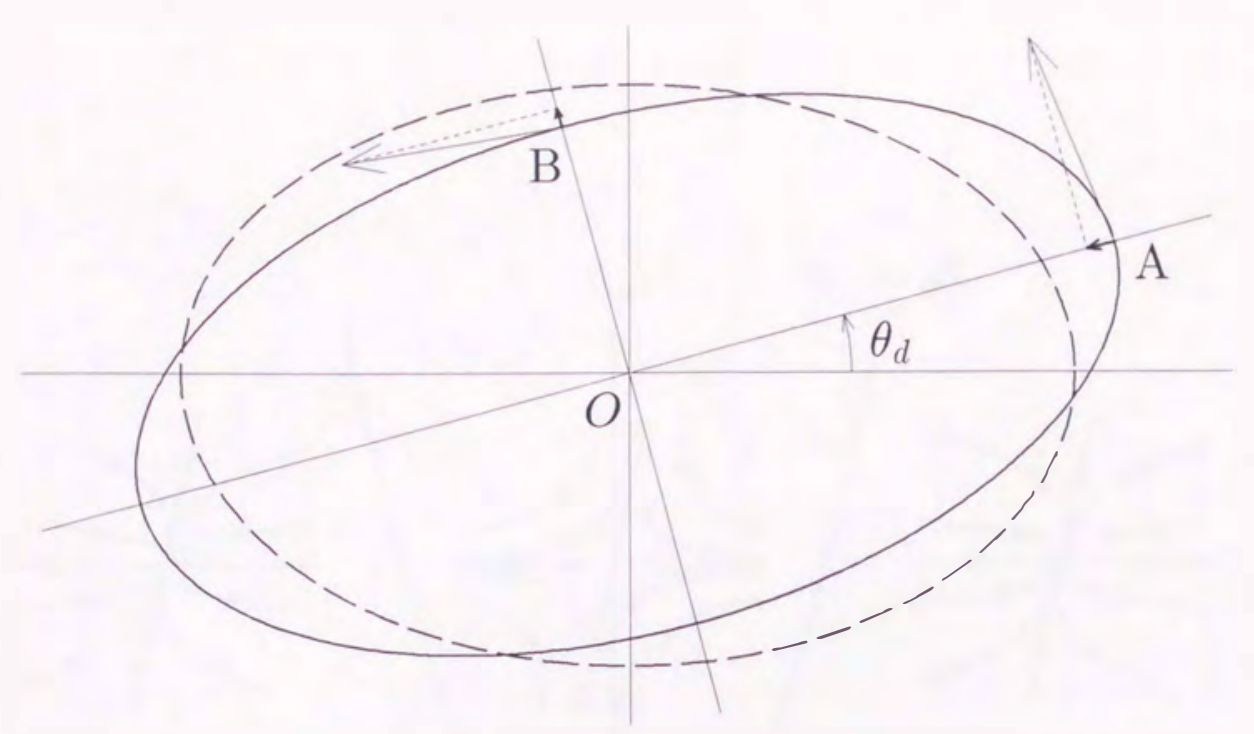

(a)

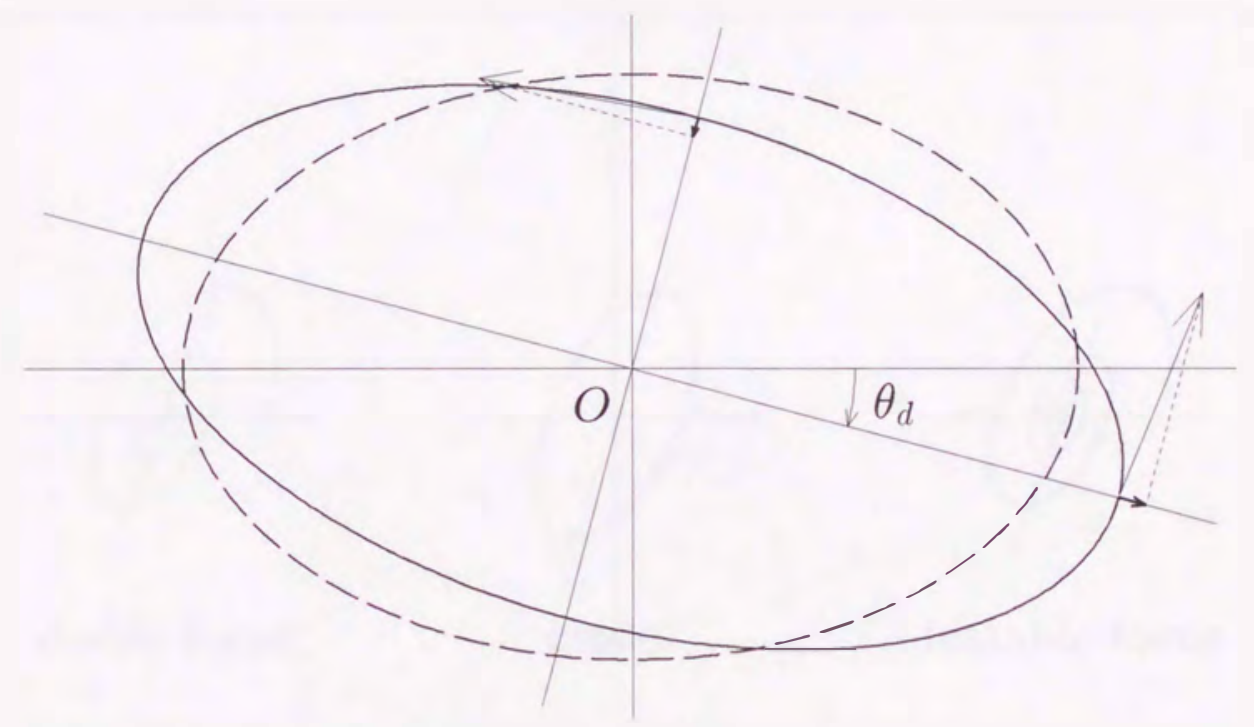

(b)

Fig. 2.7. Geometrical relation between the $q$-ellipse (solid line) and $\Psi$ ellipse (dashed line): (a) for the axisymmetrization $\left(\theta_{d}>0\right.$ ), (b) for the elongation $\left(\theta_{d}<0\right)$. The thin arrows show the velocity vectors obtained from the nearby $\Psi$ contour. The dotted lines indicate the projection of these vectors on the principal axes of the $q$-ellipse, where the thick arrows denote the projected components.

84 


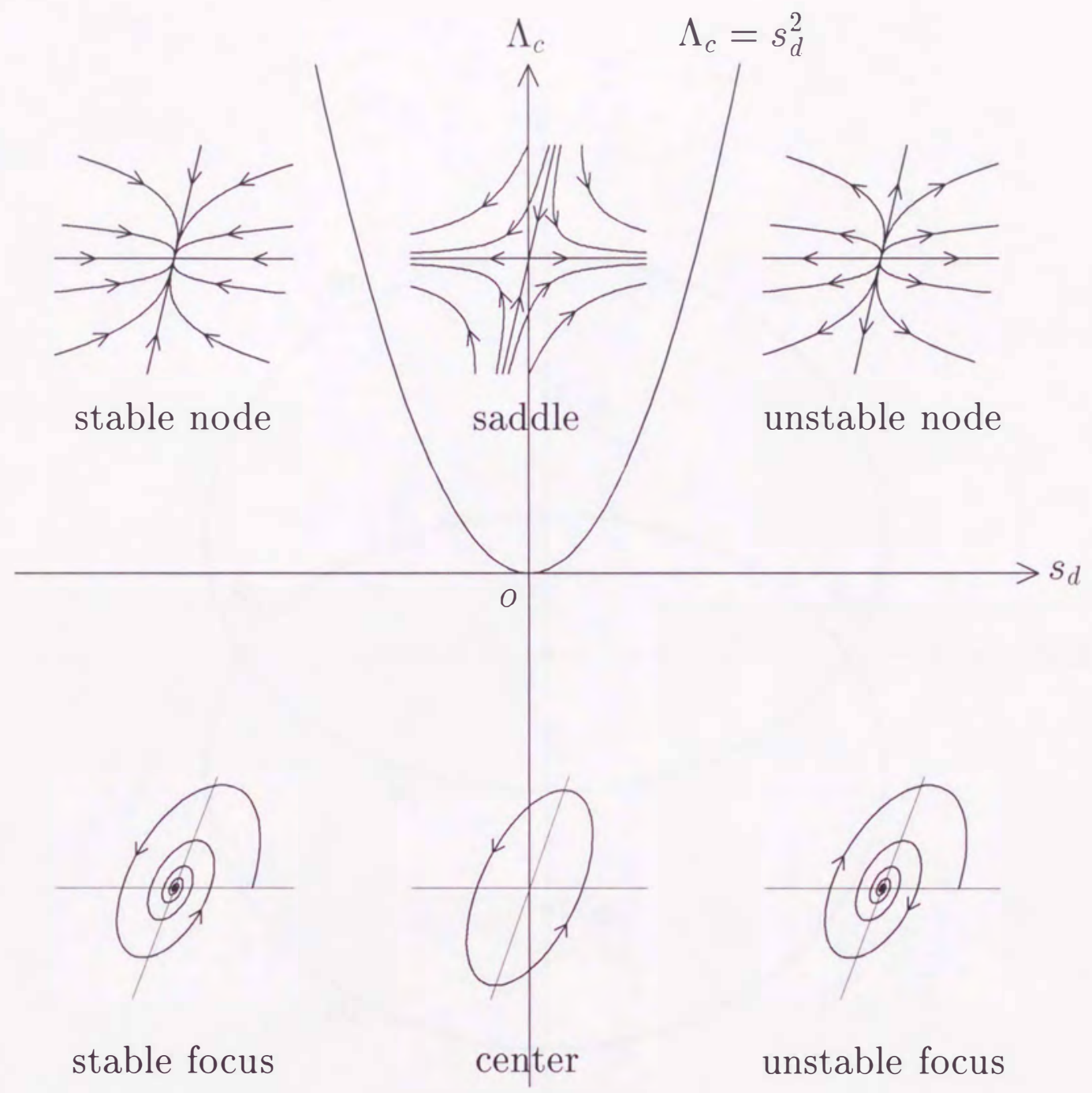

Fig. 2.8. Classification of stagnation points on the $s_{d}-\Lambda_{c}$ chart. Degenerate stagnation points, a node-saddle (when $\Lambda_{c}=s_{d}^{2}$ ) and node-focus (when $\Lambda_{c}=0$ ) are omitted here. 


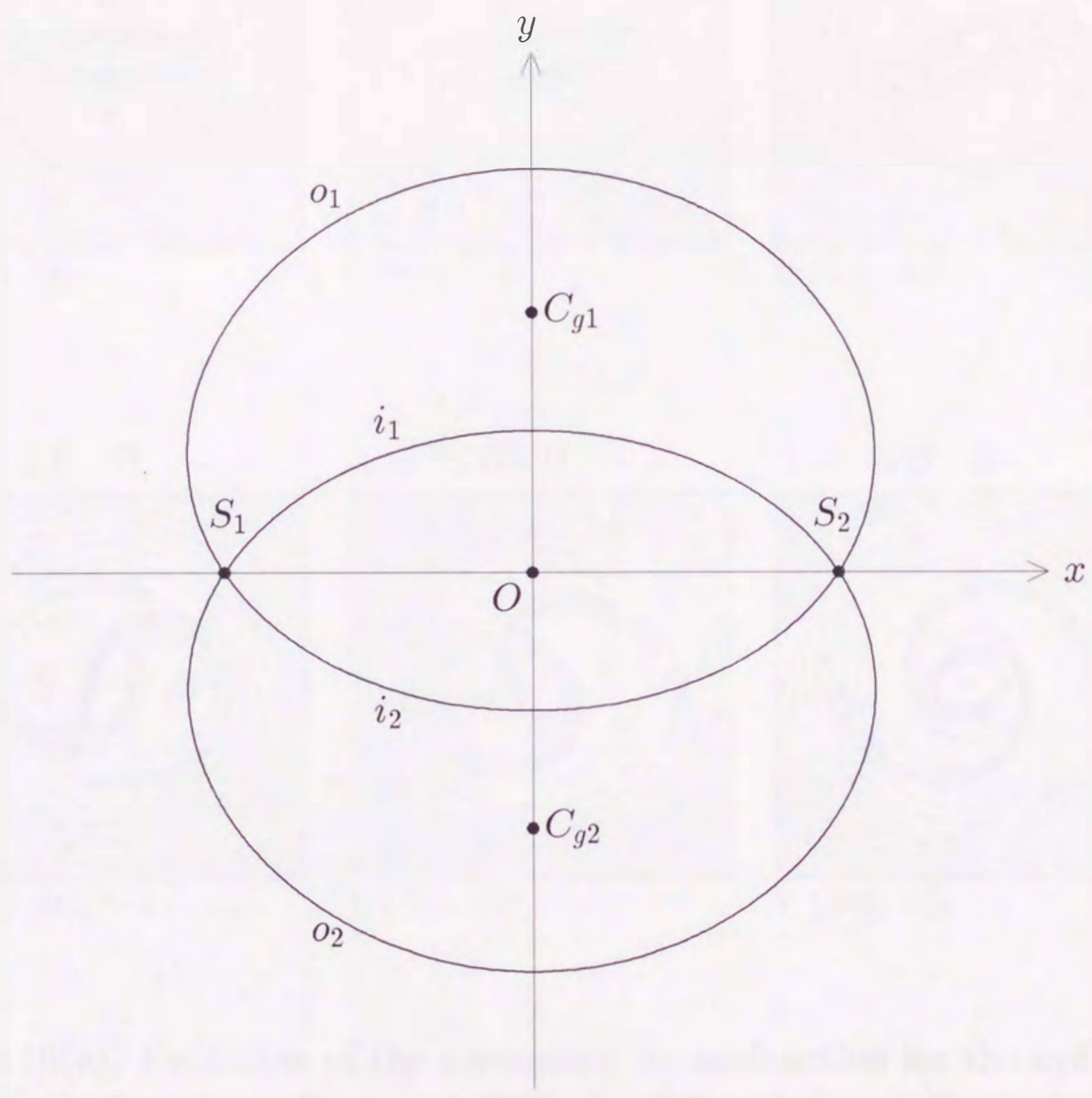

Fig. 2.9. Geometry of stagnation points for the initial elliptical vortex. $S_{1}$ and $S_{2}$ denote saddles, and $O, C_{g 1}$ and $C_{g 2}$ denote centers. The solid lines are the streamlines (called the separatrices) connecting $S_{1}$ and $S_{2}$, where $i_{1}$ and $i_{2}$ are the inner separatrices, and $o_{1}$ and $o_{2}$ are the outer separatrices. 


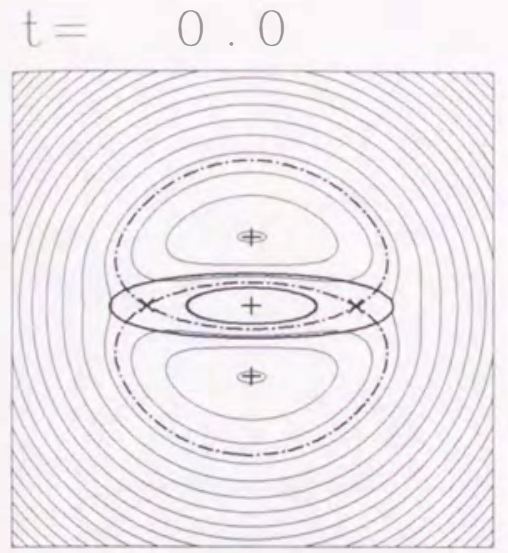

C $I=0.20$

$t=200$

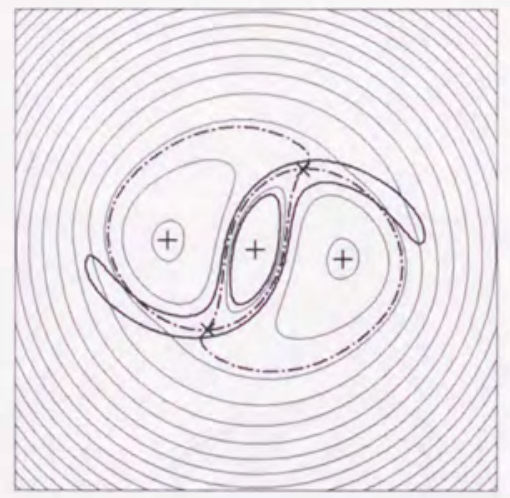

C. $I=0.25$

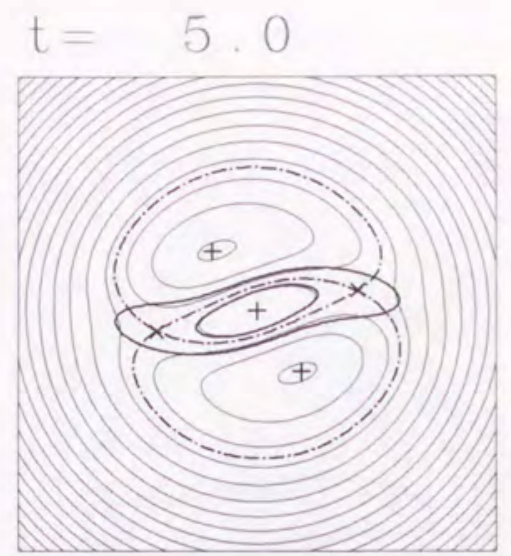

$C^{\prime} I=0.20$

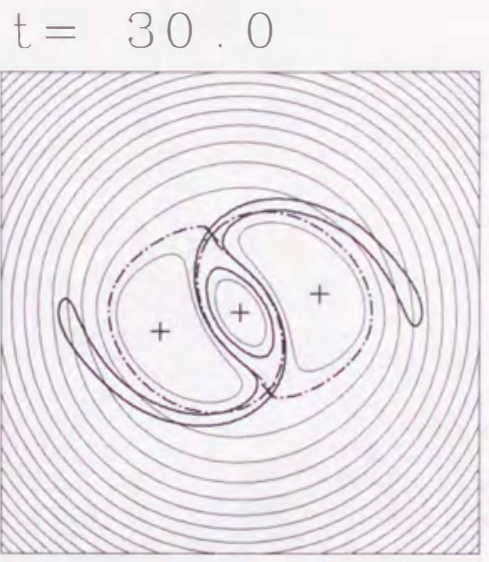

C $I=0.30$

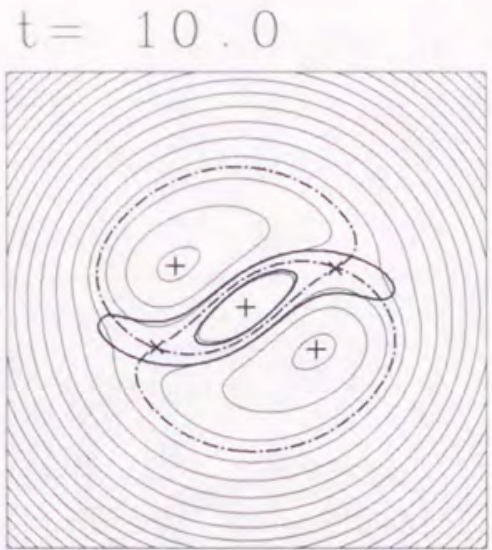

C. $I=0.20$

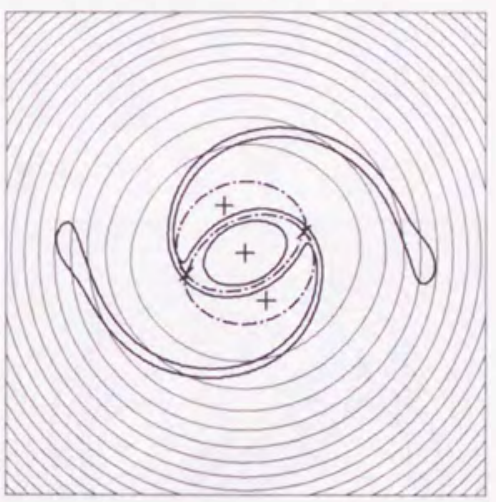

$\mathrm{C} I=0.75$

Fig. 2.10(a). Evolution of the corotating streamfunction for the cyclonic elliptical vortex with $\eta_{\max }=0.09, \lambda=2.5$ and $F=0.25 . \times$ denotes a saddle (with negative Gaussian curvature), and + denotes a center (with positive Gaussian curvature). The dash-dotted lines are the separatrices emanating from the saddle points. The inner and outer solid bold lines are the contours of potential vorticity representing the core and periphery of the vortex, respectively. The simulation was performed with $N=256$. 


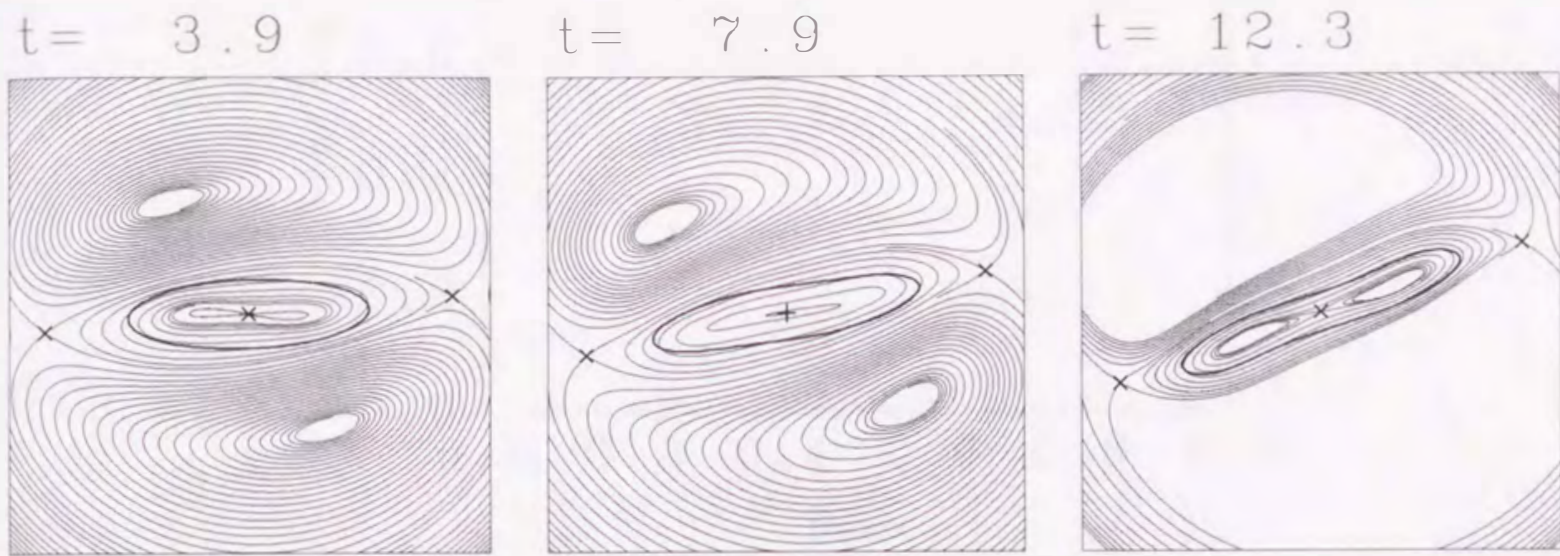

(a)
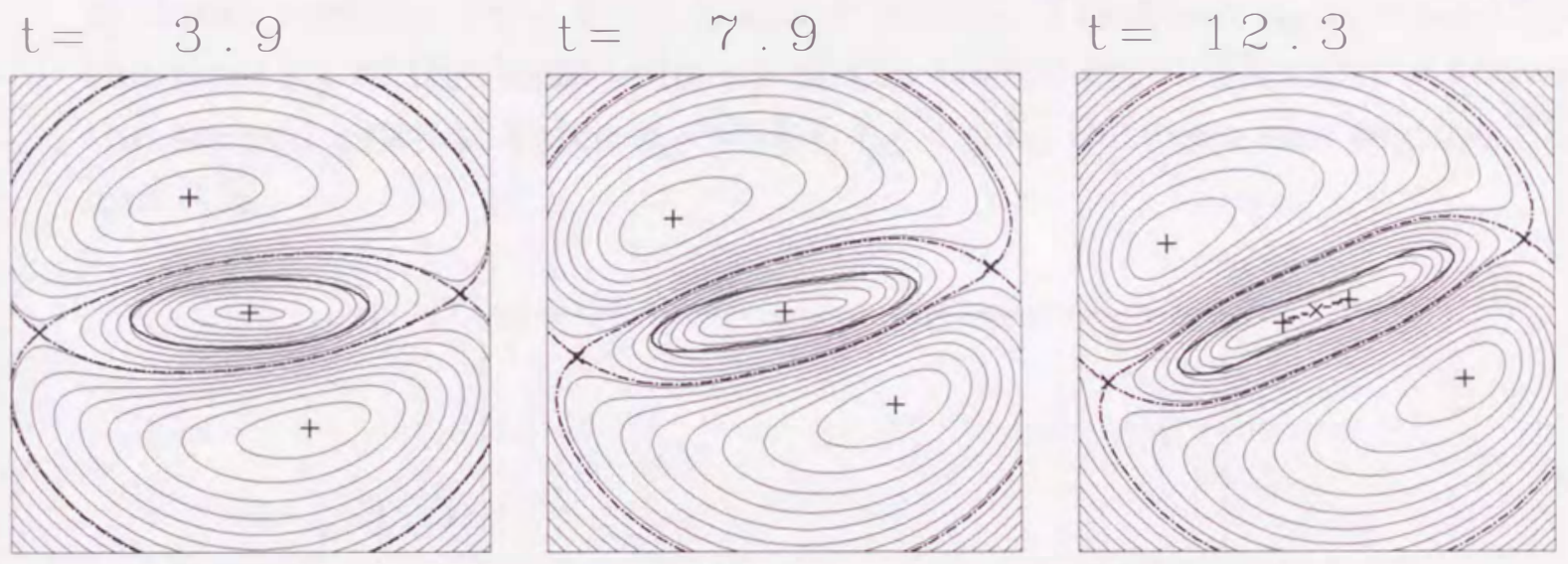

$\mathrm{C} I=0.050$

C I $=0.050$

$\mathrm{C} I=0.045$

(b)

Fig. 2.11 Flow in the corotating frame around the vortex core for the cyclonic elliptical vortex with $\eta_{\max }=0.9, \lambda=2.5$ and $F=0.25$. (a) Streamlines emanating from the saddle points denoted by $\times$. (b) Same as Fig. 2.10(b) except the region to be shown. Only the part $|x|,|y| \leq 5$ of the domain is shown. 


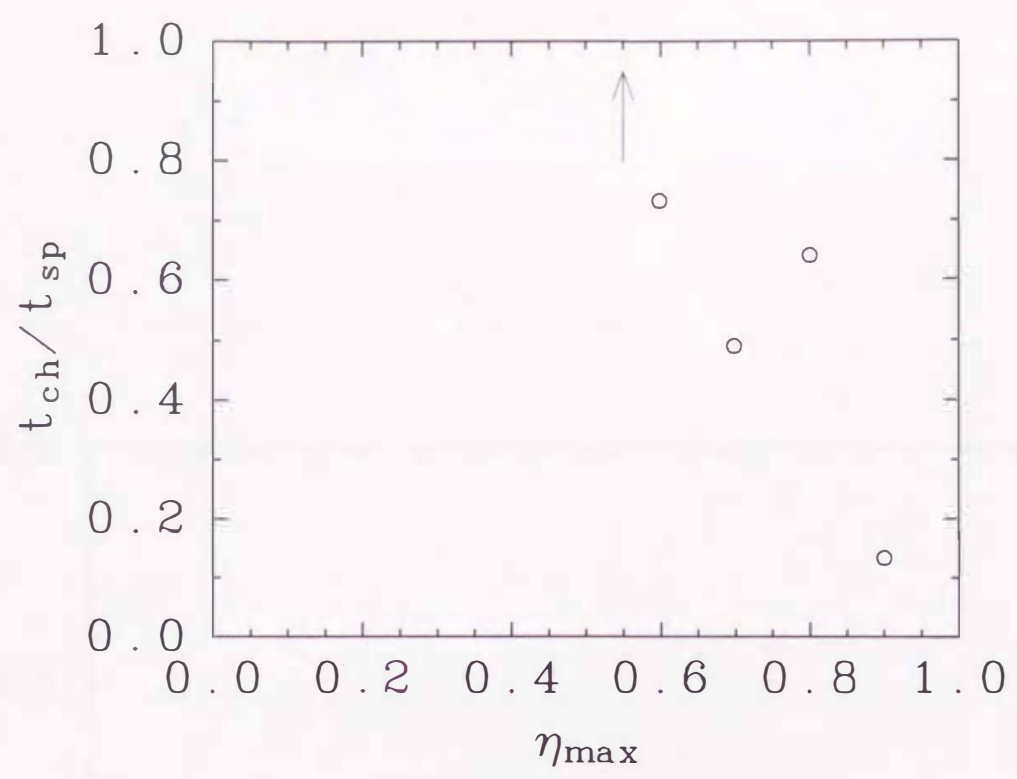

Fig. 2.12. The maximum surface displacement $\eta_{\max }$ versus the time $t_{\mathrm{ch}}$ of the change into a saddle of the stagnation point at the origin for the cyclonic vortices with $\lambda=2.5$ and $F=0.25$. The time $t_{\mathrm{ch}}$ is scaled by the time $t_{\mathrm{sp}}$ of the local split-up of the vortex core. The arrow shows the second critical value $\eta_{c 2}\left(0.5<\eta_{c 2}<0.6\right)$ between the regime SE and $\mathrm{AE}$.

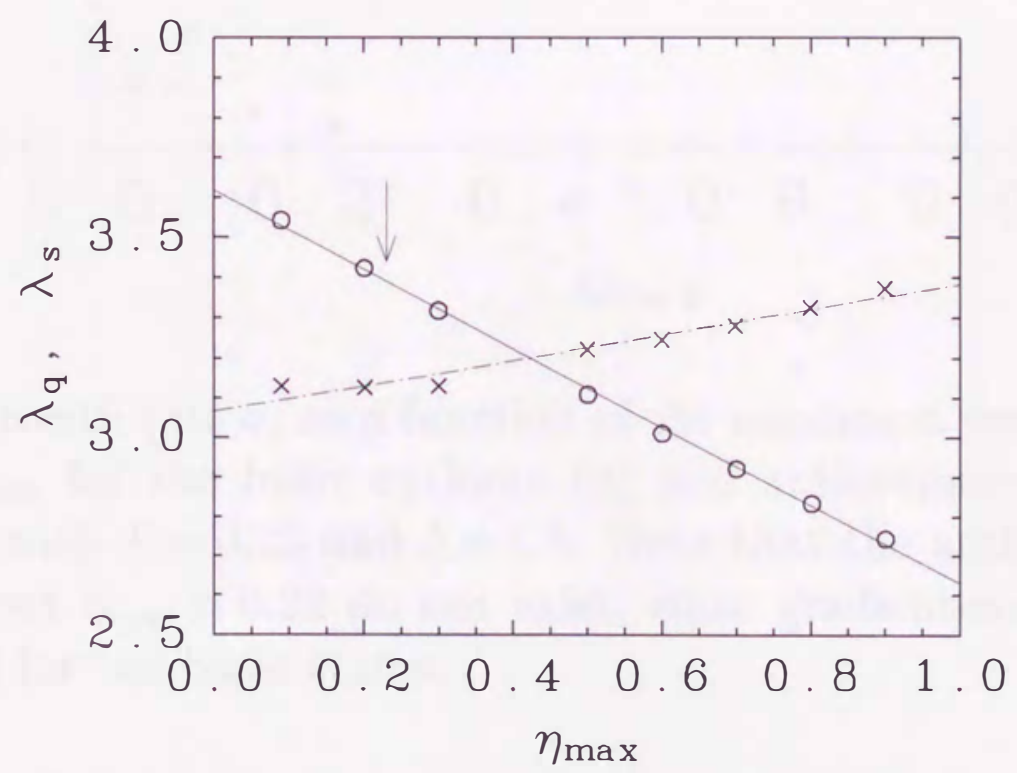

Fig. 2.13. Aspect ratios $\lambda_{q}(\circ)$ and $\lambda_{s}(\times)$ at $t=0$ as a function of the maximum surface displacement $\eta_{\max }$ for the cyclonic vortices with $\lambda=2.5$ and $F=0.25$. The arrow shows the first critical value $\eta_{c 1}=0.23$ between the regime $\mathrm{A}$ and $\mathrm{AE}$. 


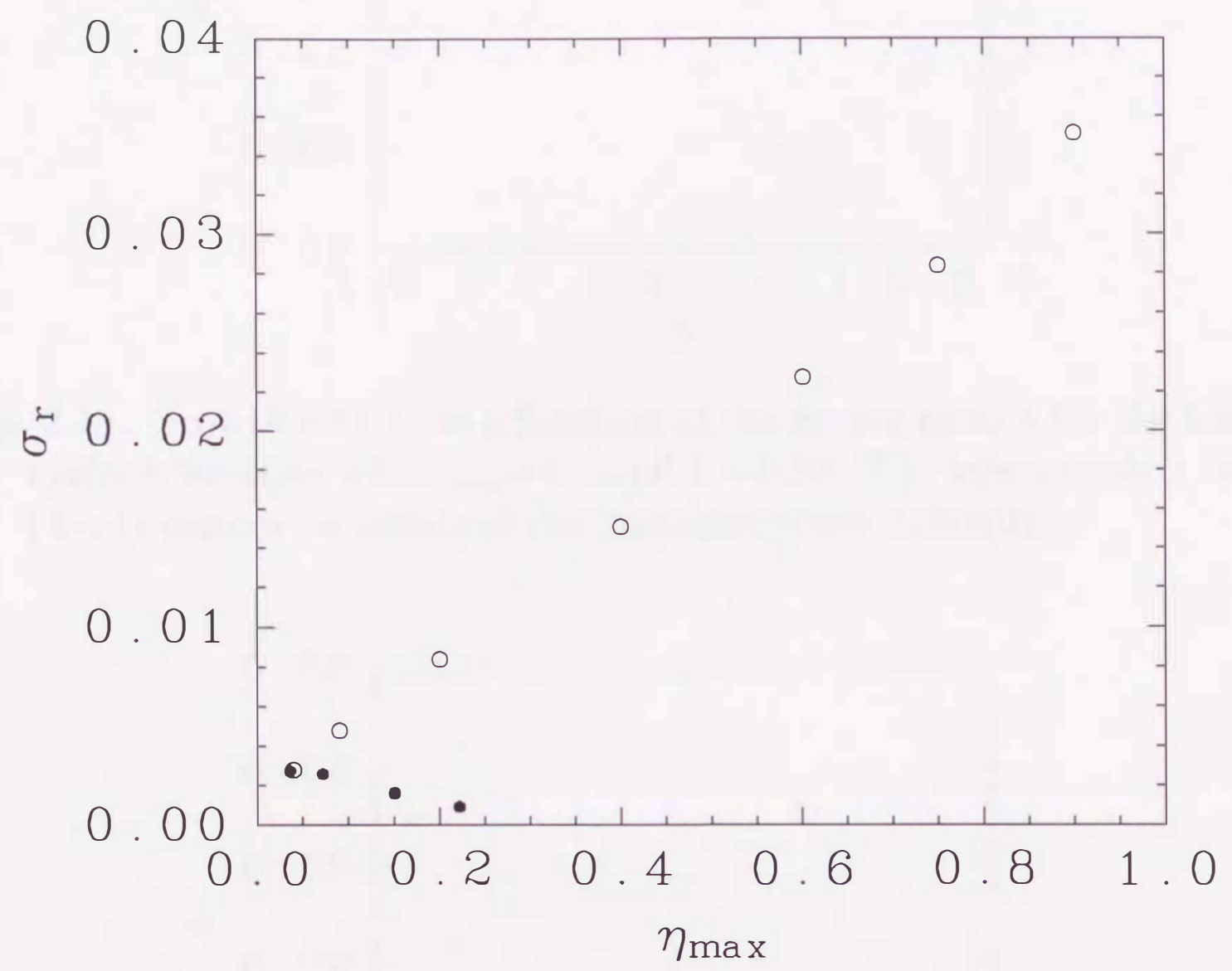

Fig. 2.14. Growth rate $\sigma_{r}$ as a function of the maximum surface displacement $\eta_{\max }$ for the basic cyclonic (०) and anticyclonic $(\bullet)$ elliptical vortices with $F=0.25$ and $\lambda=1.5$. Note that the anticyclonic cases with about $\eta_{\max }>0.22$ do not exist, since gradient-wind balance is assumed for the basic states. 


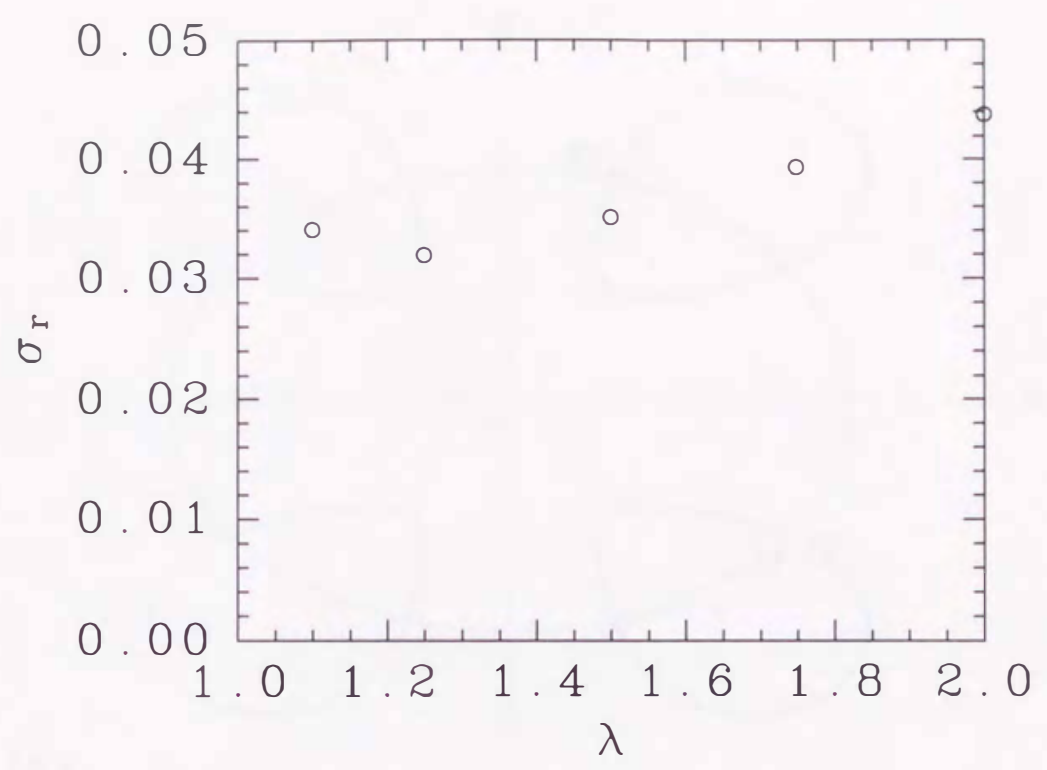

Fig. 2.15. Growth rate $\sigma_{r}$ as a function of the aspect ratio $\lambda$ for the basic cyclonic vortices with $\eta_{\max }=0.9$ and $F=0.25$. The axisymmetric case $(\lambda=1)$ cannot be obtained due to convergence difficulty.

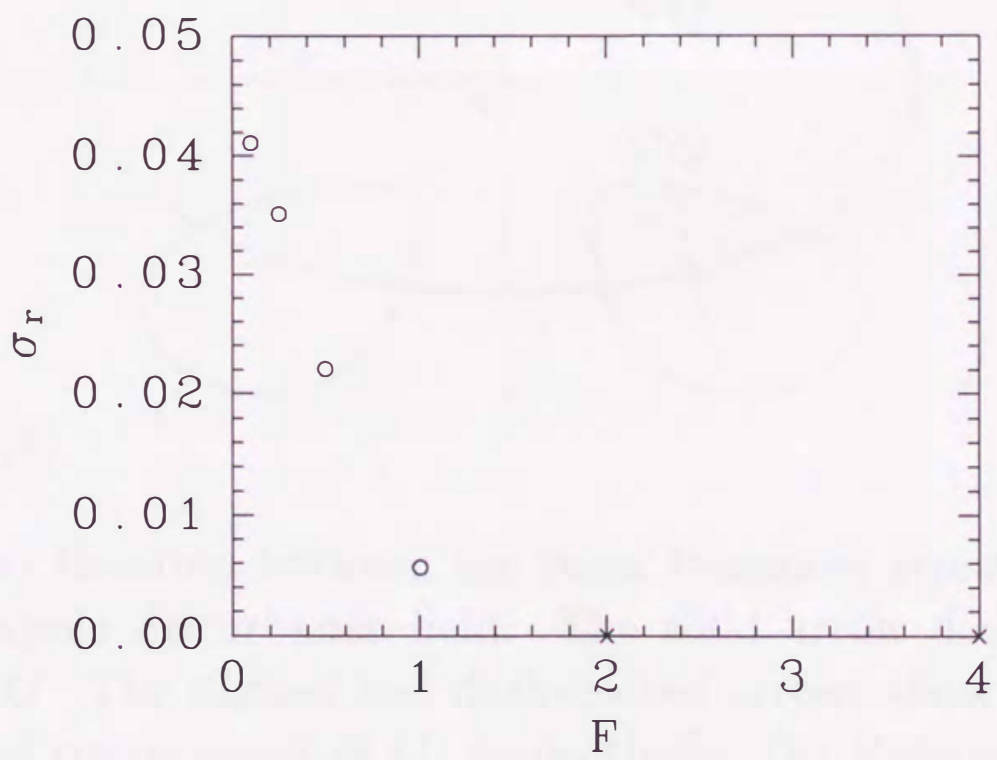

Fig. 2.16. Growth rate $\sigma_{r}$ as a function of the rotational Froude number $F$ for the basic cyclonic elliptical vortices with $\eta_{\max }=0.9$ and $\lambda=1.5$. $\circ$ denotes the presence of the unstable disturbance, while $\times$ denotes the absence of the unstable disturbance. 

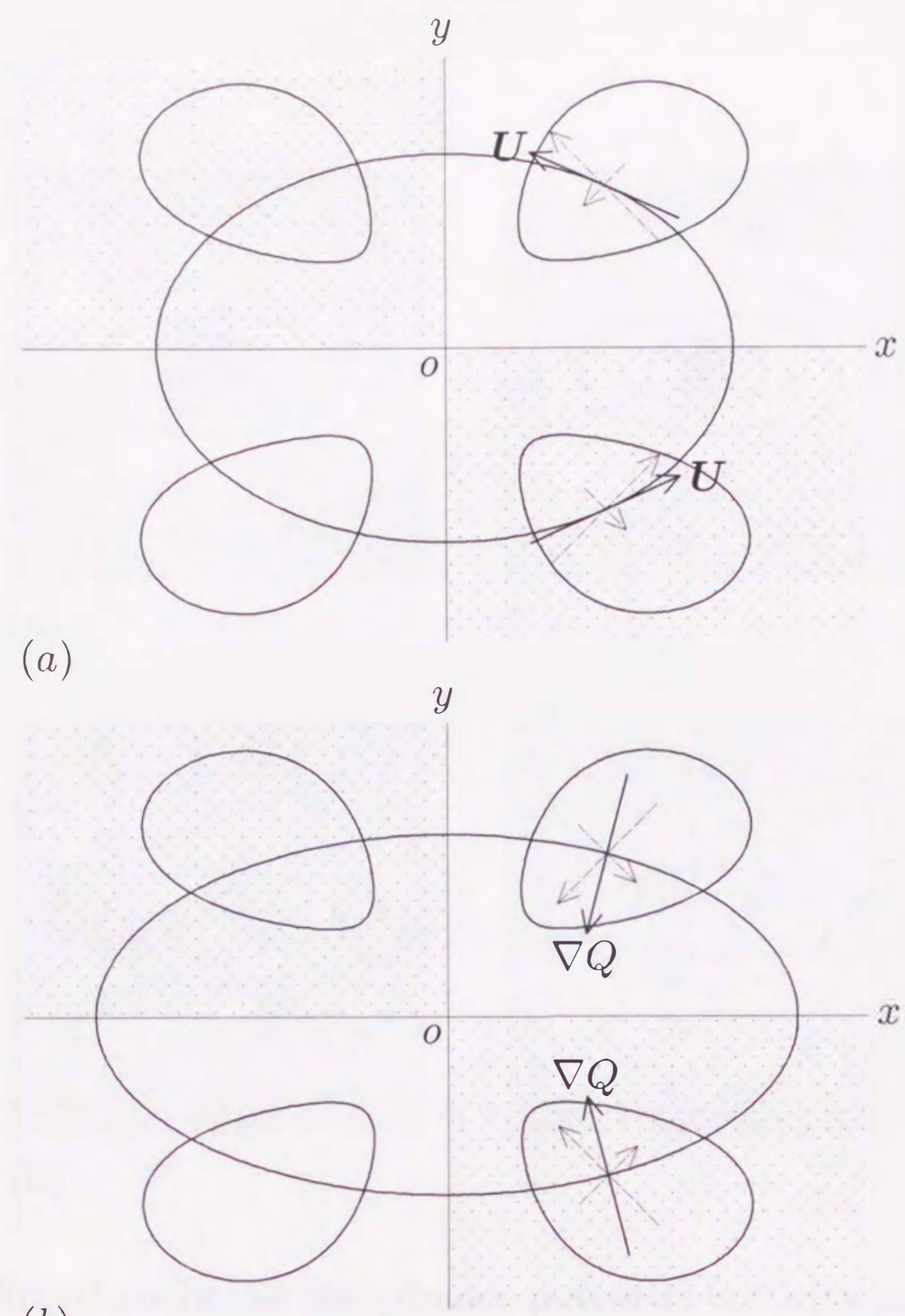

(b)

Fig. 2.17. (a) Relation between the basic transport streamfunction and a quadrupole disturbance field. The solid arrow denotes the basic velocity $\boldsymbol{U}$. The dashed and dash-dotted arrows show the radial and azimuthal components of $\boldsymbol{U}$, respectively. (b) Relation between the basic potential vorticity and the disturbance field. The solid arrow denotes the basic potential-vorticity gradient $\nabla Q$. The dashed and dash-dotted arrows show the radial and azimuthal components of $\nabla Q$, respectively. The second and fourth quadrants are shaded. 


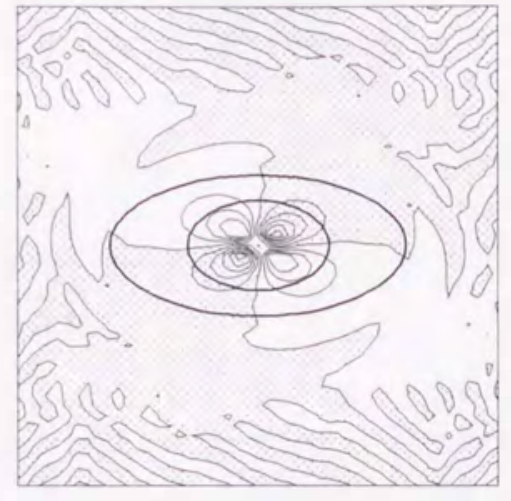

(a)
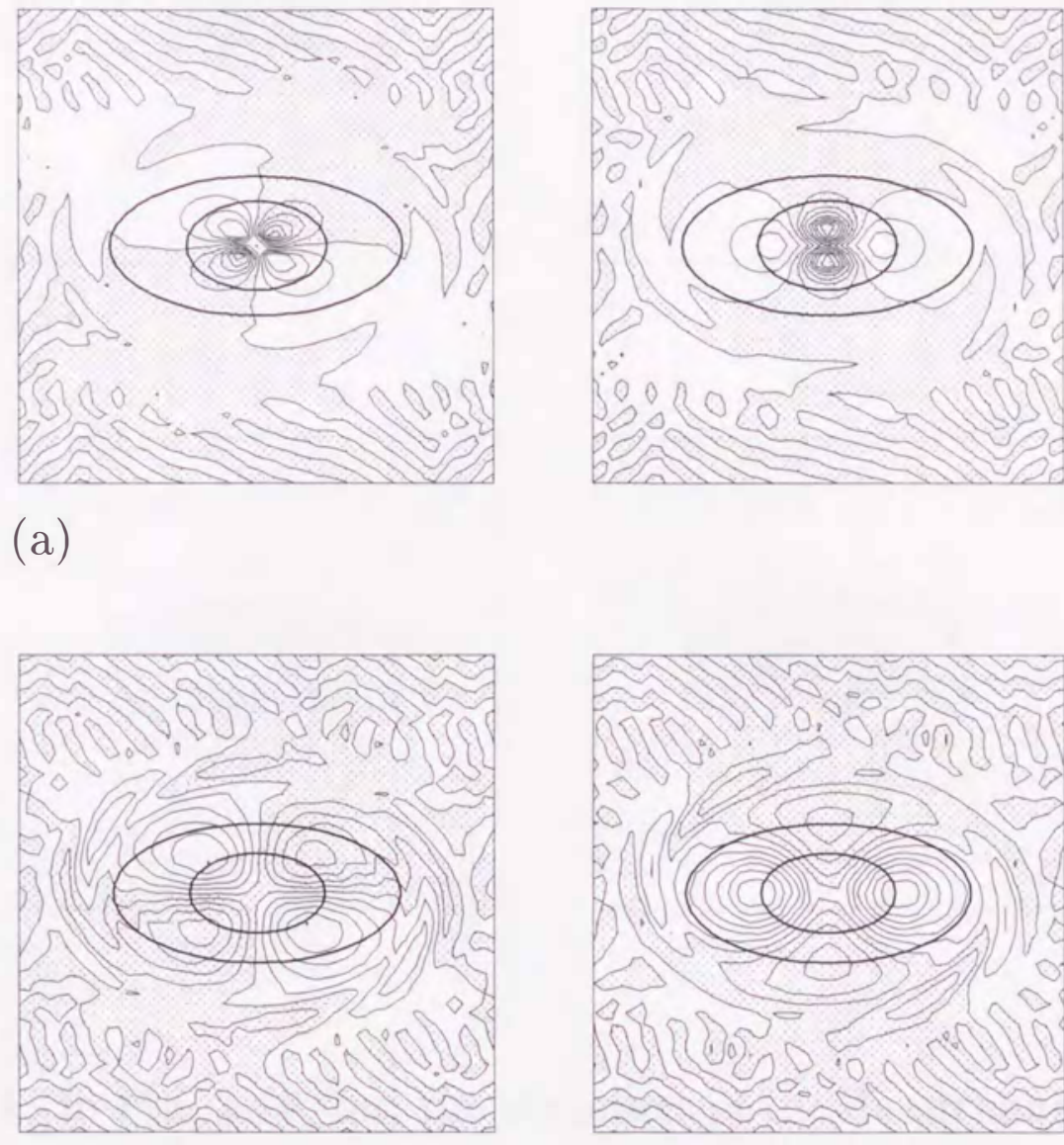

(b)

Fig. 2.18. Structure of the disturbance potential vorticity at appropriate two phases for the basic cyclonic vortices with $\lambda=1.5$ and $F=0.25$ : (a) $\eta_{\max }=0.9$, (b) $\eta_{\max }=0.4$. The phase of the disturbance shown in the right panel proceeds $90^{\circ}$ relative to that in the left panel. The contour interval is arbitrary. The regions of negative values are shaded. The inner and outer bold lines are the contours of the basic potential vorticity representing the core and periphery, respectively. Only the part $|x|,|y| \leq 4.5$ of the domain is shown. 


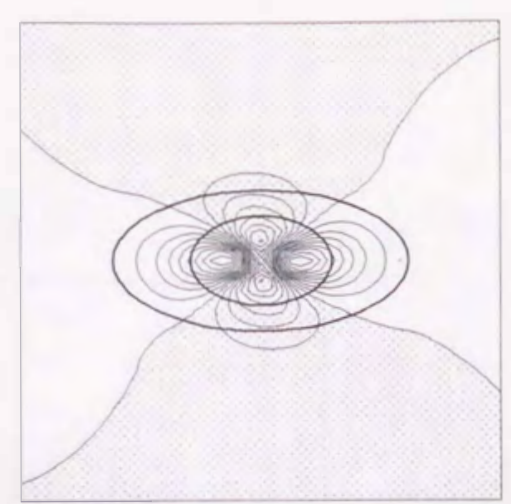

(a)
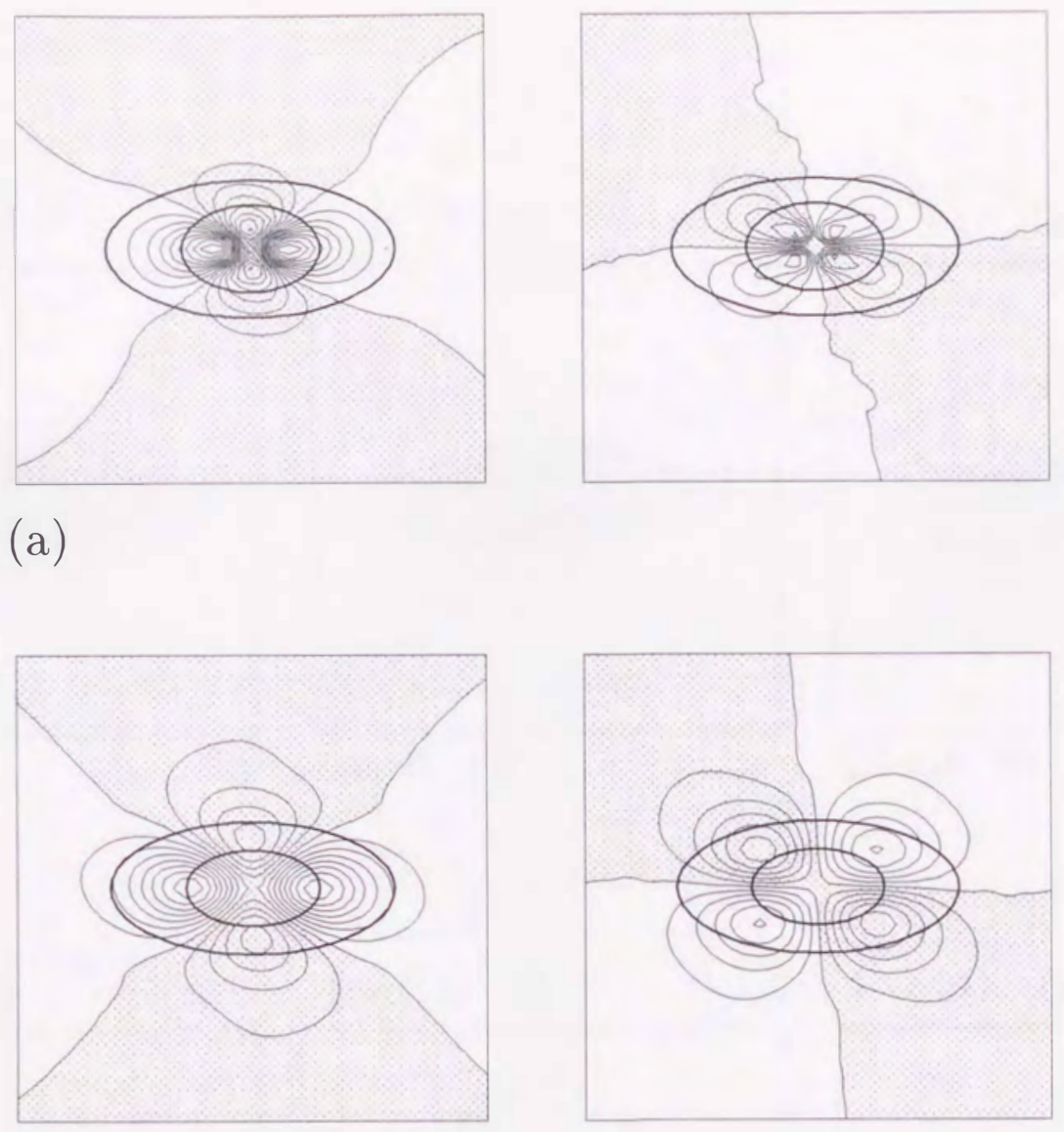

(b)

Fig. 2.19. As in Fig. 2.18, but the structure of the disturbance divergence $\nabla \cdot u$.

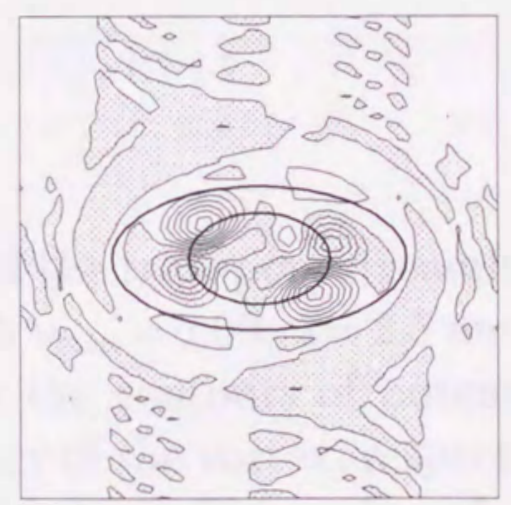

Fig. 2.20. As in Fig. 2.18, but the distribution of the energy transfer rate from the basic to disturbance field averaged over one cycle for the basic cyclonic vortex with $\eta_{\max }=0.9, \lambda=1.5$ and $F=0.25$. 


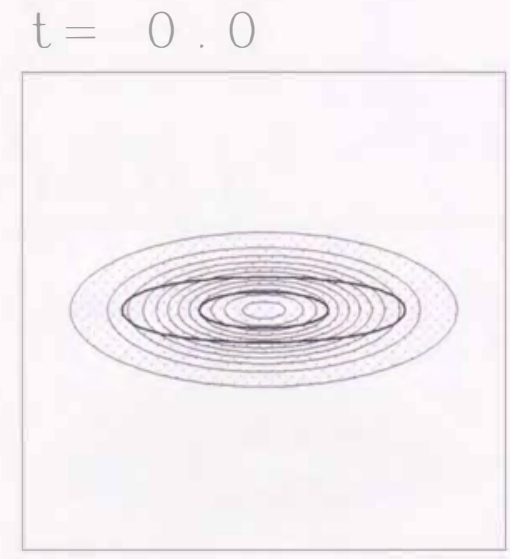

$\mathrm{C} I=0.08$

\section{$\mathrm{t}=20$}

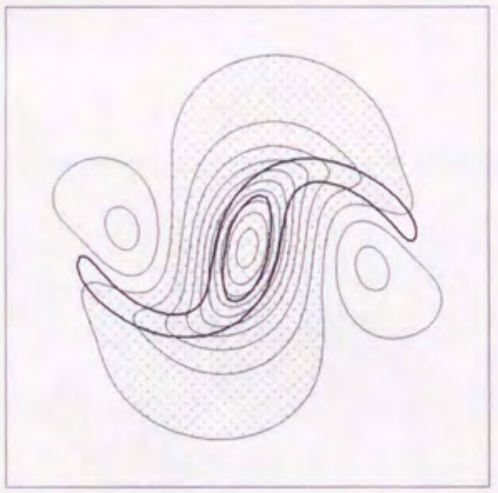

$\mathrm{C} I=0.10$

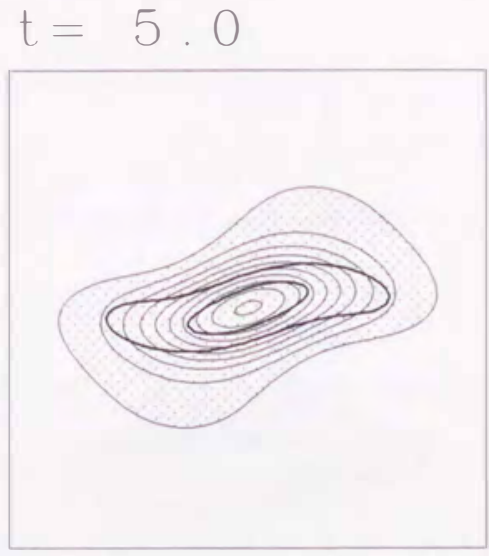

$\mathrm{C} I=0.10$

$t=30$

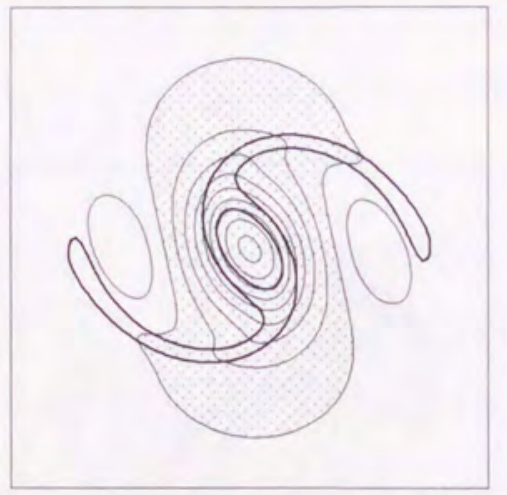

$\mathrm{C} I=0.12$ $t=10.0$

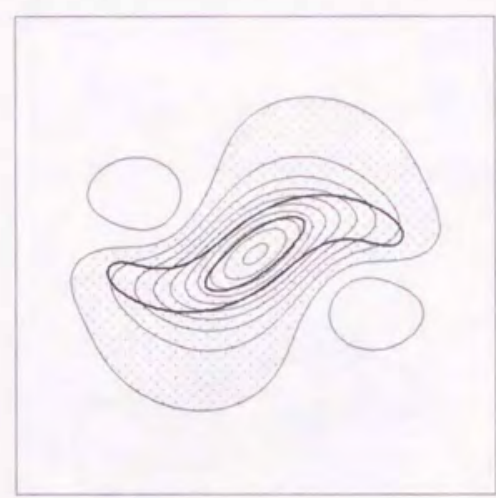

C I $=0.10$ $t=40.0$

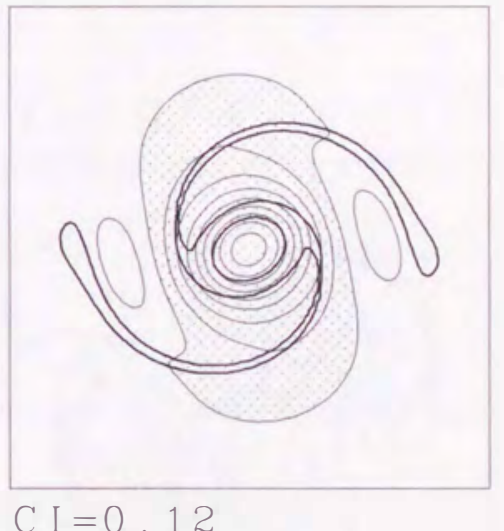

Fig. 2.C(a). Evolution of the transport streamfunction $\Psi$ for the cyclonic elliptical vortex with $\eta_{\max }=0.09, \lambda=2.5$ and $F=0.25$. The inner and outer bold lines are the contours of potential vorticity representing the core and periphery of the vortex, respectively. The spatial average of $\Psi$ is assumed to be zero. The region of negative values is shaded. The interval of contours is denoted by CI below each panel. 



\section{Acknowledgements}

The author is greatly indebted to his thesis advisor, Prof. T. Yamagata for bringing the present problem to his attention, and for helpful discussions and encouragement. He is also grateful to Prof. S. Miyahara and Prof. O. Morita for their careful reading of the manuscript. He also acknowledges the fellowship offered by the Japan Society for the Promotion of Science from April 1992 to March 1994. The present work was done during his stay at Department of Earth and Planetary Physics of University of Tokyo from October 1991 to June 1994. He wishes to thank Dr. N. Maximenko for useful discussions and encouragement during the stay at this university as a post-doctoral fellow. Main part of the computations were made on the workstations of SUN SPARC station ELC and SPARC station 2, using fast Fourier transforms in the SSL II library. Slight part of the computations including the linear stability analysis were made on the HITAC S-3800 supercomputer at Computational Center of University of Tokyo, using fast Fourier transforms and eigenvalue routines in the MATRIX/HAP library. He also thanks the staffs and graduate students of the fluid geophysics groups of University of Tokyo for providing the convenient computational environment. All figures were drawn by GFD-DENNOU Library. 


\section{References}

M. E. Brachet, M. Meneguzzi, H. Politano, and P. L. Sulem, The dynamics of freely decaying two-dimensional turbulence, J. Fluid Mech. 194, 333-349 (1988).

B. Cushman-Roisin, Frontal geostrophic dynamics, J. Phys. Oceanogr. 16, 132-143 (1986).

B. Cushman-Roisin, Linear stability of large, elliptical warm-core rings, J. Phys. Oceanogr. 16, 1158-1164 (1986).

B. Cushman-Roisin, W. H. Heil, and D. Nof, Oscillations and rotations of elliptical warm-core rings, J. Geophys. Res. 90, 11756-11764 (1985).

B. Cushman-Roisin and B. Tang, Geostrophic regimes and geostrophic turbulence beyond the radius of deformation, Mesoscale/Synoptic Coherent Structures in Geophysical Turbulence, J. C. J. Nihoul and B. M. Jamart, Eds., Elsevier Oceanogr. Ser. 50, 51-74 (1989).

B. Cushman-Roisin and B. Tang, Geostrophic turbulence and emergence of eddies beyond the radius of deformation, J. Phys. Oceanogr. 20, 97-113 (1990).

D. G. Dritschel, The nonlinear evolution of rotating configurations of uniform vorticity, J. Fluid Mech. 172, 157-182 (1986).

M. Farge and R. Sadourny, Wave-vortex dynamics in rotating shallow water, J. Fluid Mech. 206, 433-462 (1989).

G. R. Flierl, On the instability of geostrophic vortices, J. Fluid Mech. 197, 349-388 (1988).

P. R. Gent and J. C. McWilliams, Consistent balanced models in bounded and periodic domains, Dyn. Atmos. Oceans 7, 67-93 (1983).

R. W. Griffiths, P. D. Killworth, and M. E. Stern, Ageostrophic instability of ocean currents, J. Fluid Mech. 117, 343-377 (1982).

H. Hukuda and T. Yamagata, A unified geostrophic equation with appli- 
cation to a cold core ring, Tellus 40A, 407-418 (1988).

R. H. Kraichnan and D. Montgomery, Two-dimensional turbulence, Rep. Prog. Phys. 43, 547-619 (1980).

S. H. Lamb, Hydrodynamics, Cambridge University Press, New York (1932).

V. D. Larichev and J. C. McWilliams, Weakly decaying turbulence in an equivalent-barotropic fluid, Phys. Fluids A 3, 938-950 (1991).

J. C. McWilliams, The emergence of isolated coherent vortices in turbulent flow, J. Fluid Mech. 146, 21-43 (1984).

J. C. McWilliams, Submesoscale, coherent vortices in the ocean, J. Geophys. Res. 23, 165-182 (1985).

J. C. McWilliams, The vortices of two-dimensional turbulence, J. Fluid Mech. 219, 361-385 (1990).

M. V. Melander, J. C. McWilliams, and N. J. Zabusky, Axisymmetrization and vorticity-gradient intensification of an isolated two-dimensional vortex through filamentation, J. Fluid Mech. 178, 137-159 (1987).

N. J. Norton, J. C. McWilliams, and P. R. Gent, A numerical model of the balance equations in a periodic domain and an example of balanced turbulence, J. Comp. Phys. 67, 439-471 (1986).

E. G. Pavia and B. Cushman-Roisin, Modeling of oceanic fronts using a particle method, J. Geophys. Res. 93, 3554-3562 (1988).

E. G. Pavia and B. Cushman-Roisin, Merging of frontal eddies, J. Phys. Oceanogr. 20, 1886-1906 (1990).

J. Pedlosky, Geophysical fluid dynamics, Springer-Verlag, New York (1987).

A. E. Perry and M. S. Chong, A description of eddying motions and flow patterns using critical-point concepts, Ann. Rev. Fluid Mech. 19, 125-155 (1987). 
L. M. Polvani, G. R. Flierl, and N. J. Zabusky, Filamentation of unstable vortex structures via separatrix crossing: A quantitative estimate of onset time, Phys. Fluids A 1, 181-184 (1989).

L. M. Polvani, N. J. Zabusky, and G.R. Flierl, Two-layer geostrophic vortex dynamics. Part 1. Upper-layer V-states and merger, J. fluid Mech. 205, 215-242 (1989).

P. Ripa, General stability conditions for zonal flows in a one-layer model on the $\beta$-plane or the sphere, J. Fluid Mech. 126, 463-489 (1983).

A. R. Robinson, Eddies in Marine Science, Springer-Verlag, New York (1983).

P. Santangelo, R. Benzi, and B. Legras, The generation of vortices in highresolution, two-dimensional decaying turbulence and the influence of initial conditions on the breaking of self-similarity, Phys. Fluids A 1, 1027-1034 (1989).

C. Sulem, P. L. Sulem, and H. Frisch, Tracing complex singularities with spectral methods, J. Comp. Phys. 50, 138-161 (1983).

J. Weiss, The dynamics of enstrophy transfer in two-dimensional hydrodynamics, Physica D 48, 273-294 (1991).

G. P. Williams and T. Yamagata, Geostrophic regimes, intermediate solitary vortices and Jovian eddies, J. Atom. Sci. 41, 453-478 (1984).

T. Yamagata, On nonlinear planetary waves: A class of solutions missed by the traditional quasi-geostrophic approximation, J. Oceanogr. Soc. Japan 38, 236-244 (1982). 


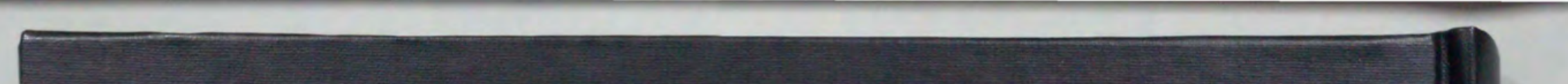




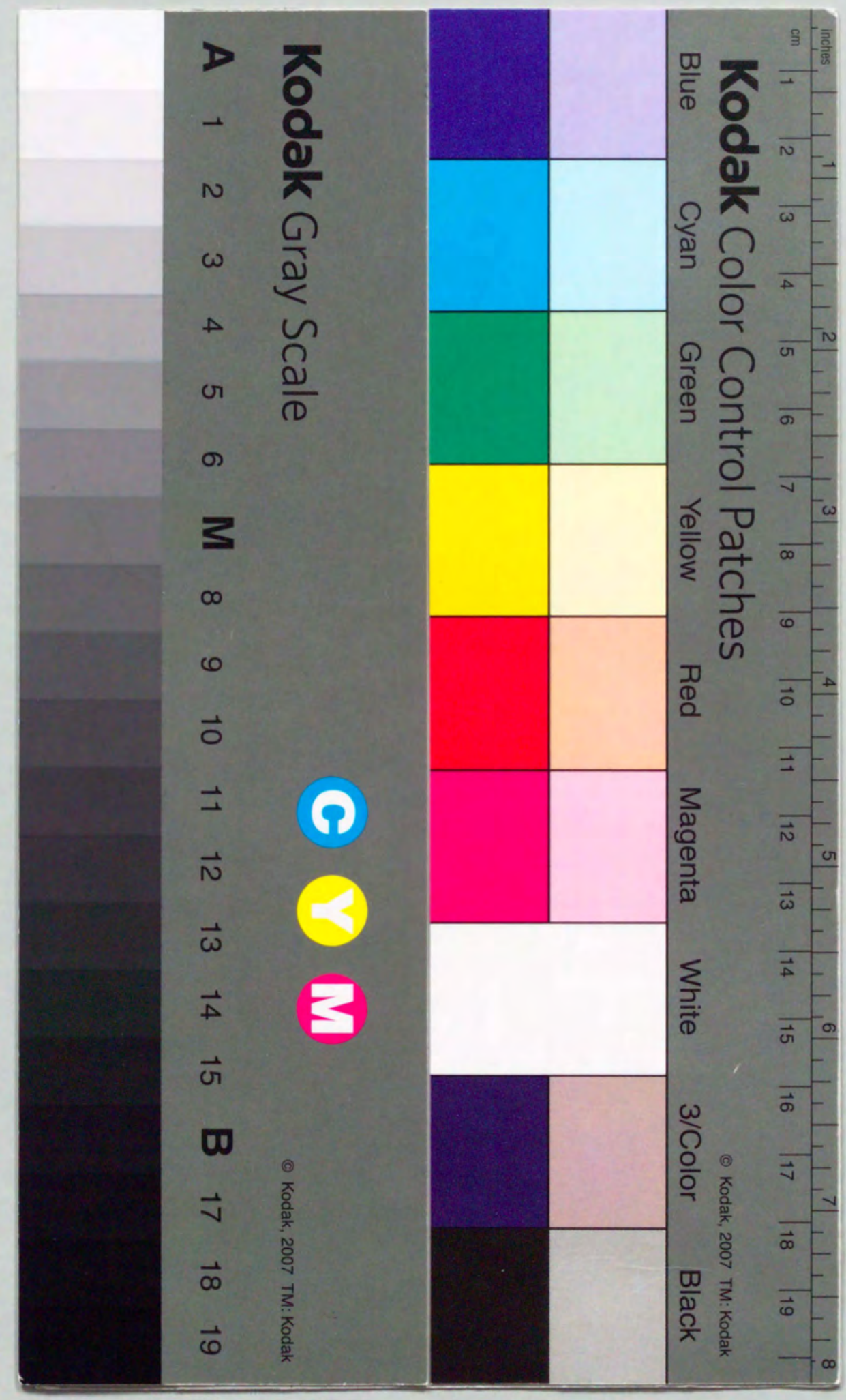

